\title{
THE ARITHMETIC OF DISTRIBUTIONS IN FREE PROBABILITY THEORY
}

\author{
G. P. CHISTYAKOV ${ }^{1,3}$ AND F. GÖTZE ${ }^{2,3}$
}

\begin{abstract}
We give a new approach to the definition of additive and multiplicative free convolutions which is based on the theory of Nevanlinna and of Schur functions. We consider the set of probability distributions as a semigroup $\mathbf{M}$ equipped with the operation of free convolution and prove a Khintchine type theorem for factorization of elements of this semigroup. Any element of $\mathbf{M}$ contains either indecomposable ("prime") factors or it belongs to a class, say $I_{0}$, of distributions without indecomposable factors. In contrast to the classical convolution semigroup in the free additive and multiplicative convolution semigroups the class $I_{0}$ consists of units (i.e. Dirac measures) only. Furthermore we show that the set of indecomposable elements is dense in $\mathbf{M}$.
\end{abstract}

\section{INTRODUCTION}

In recent years a larger number of papers has been devoted to applications and extensions of the definition of free convolution of measures introduced by D. Voiculescu. The key concept of this definition is the notion of freeness, which can be interpretated as a kind of independence for noncommutative random variables. As in the classical probability where the concept of independence gives rise to the classical convolution, the concept of freeness leads to a binary operation on the probability measures on the real line, called free convolution. As one might expect there are many classical results for sums of independent random variables having a counterpart in this theory, such as the law of large numbers, the central limit theorem, the Lévy-Khintchine formula and others. We refer to Voiculescu, Dykema and Nica [29] (1992) for an introduction to these topics. One of the main problems in dealing with free convolution is that its definition is rather indirect. In the first part of this paper we propose a new approach to the definition of additive and multiplicative free convolution. This approach is based on the classical theory of Nevanlinna and Schur functions and allows us to give a direct definition of free convolutions (see Theorem 2.1, Theorem 2.4, Theorem 2.7).

In the second part of the paper we study the arithmetic structure of the Voiculescu semigroups $(\mathcal{M}, \boxplus),\left(\mathcal{M}_{+}, \otimes\right)$, and $\left(\mathcal{M}_{*}, \otimes\right)$ of probability measures on $\mathbb{R}$

\footnotetext{
${ }^{3}$ Research supported by DFG - Forschergruppe FOR 399/2. Partly supported by INTAS grant N 03-51-5018.
} 
with additive free convolution $(\boxplus)$, on $\mathbb{R}_{+}$and on $\mathbb{T}$ with multiplicative free convolution $(\otimes)$, respectively. This subject had its origin in the work of Khintchine on the convolution semigroup $(\mathcal{M}, *)$ of probability measures on the real line. He derived for this semigroup the three basic theorems listed in Section 2. Kendall [13] (1967), 14] (1968) introduced the so called Delphic semigroups, which are commutative topological semigroups satisfying the central limit theorem for triangular arrays. Their arithmetic is similar to the convolution arithmetic of probability measures on $\mathbb{R}$. A characteristic feature of all these semigroups is the presence of infinitely divisible (i.d.) elements, which for every positive integer $n$ may be represented as $n$th power of some element of the semigroup.

In any Delphic semigroup there are three classes of its elements:

- the indecomposable or simple elements, which have no factors besides themselves and the identity, (a set we shall denote by " $S$ ");

- the elements which are decomposable and have an indecomposable factor, (a set we shall denote by " $D$ ");

- the infinitely divisible elements which have no indecomposable factors, (a set we shall denote by " $I_{0}$ ").

It turns out a lot of important semigroups, in particular $(\mathcal{M}, *)$, are Delphic or almost Delphic.

It is convenient to formulate the Delphic hypothesis rather restrictively, and then to show that semigroups like $(\mathcal{M}, *)$ are 'almost' Delphic, that means they satisfy these hypothesis with nonessential modifications. In this context Davidson [9]-11 (1968), (1969) introduced the concept of an hereditary subsemigroup to verify that semigroups are almost (or properly) Delphic. Using a new multivariate analytic description of free convolutions we show that the Voiculescu semigroups $(\mathcal{M}, \boxplus),\left(\mathcal{M}_{+}, \otimes\right)$, and $\left(\mathcal{M}_{*}, \otimes\right)$ are almost Delphic semigroups. Using some ideas of Kendall and Davidson, we deduce the three basic Delphic theorems for these semigroups. One of them states that each element of a Delphic semigroup may be written as a product of a countable number of indecomposable elements and an element of $I_{0}$, so a knowledge of $I_{0}$ and the set of indecomposable elements is essential for the arithmetic of the semigroup. As a consequence we show that in the Voiculescu semigroups the class $I_{0}$ consists of Dirac measures $\delta_{a}$ only and for each semigroup there is a dense set of indecomposable elements.

As another consequence of this approach we obtain an analogue of Khintchine's limit theorem for triangular arrays and derive the Lévy-Khintchine formula for i.d. probability measures in Voiculescu's semigroups.

The paper is organized as follows. In Section 2 we discuss the results of the paper. In Section 3 we collect auxiliary results from complex analysis, free probability, and Delphic semigroups. In Sections 4 and 5 we prove the necessary analytical results for our approach to free convolutions. In Section 6 we prove basic Delphic theorems for the Voiculecu semigroups, in Section 7 we describe the class $I_{0}$, 
and in Section 8 we describe dense classes of indecomposable elements in these semigroups.

\section{Results}

Denote by $\mathcal{M}$ the family of all Borel probability measures (p-measures for short) defined on the real line $\mathbb{R}$. On the set $\mathcal{M}$ there are defined two associative composition laws denoted $*$ and $\boxplus$. Let $\mu_{1}, \mu_{2} \in \mathcal{M}$. The measure $\mu_{1} * \mu_{2}$ is the classical convolution of $\mu_{1}$ and $\mu_{2}$. In probabilistic terms, $\mu_{1} * \mu_{2}$ is the probability distribution of $X+Y$, where $X$ and $Y$ are (commuting) independent random variables with distributions $\mu_{1}$ and $\mu_{2}$ respectively. The measure $\mu_{1} \boxplus \mu_{2}$ denote the free (additive) convolution of $\mu_{1}$ and $\mu_{2}$ introduced by Voiculescu [27. (1986) for compactly supported measures. The notion of free convolution was extended by Maassen [18] (1992) to measures with finite variance and by Bercovici and Voiculescu [4] (1993) to all measures in $\mathcal{M}$. Here, $\mu_{1} \boxplus \mu_{2}$ may be considered as the probability distribution of $X+Y$, where $X$ and $Y$ are free random variables with distributions $\mu_{1}$ and $\mu_{2}$, respectively. For positive random variables and for random variables with values on $\mathbb{T}$ we consider multiplicative convolutions as well and their free analogues of multiplicative convolutions which were introduced by Voiculescu [28] (1987).

In this section we give a new approach to the definition of $\mu_{1} \boxplus \mu_{2}$ which extends Maassen's definition. Furthermore, we shall present a new approach to

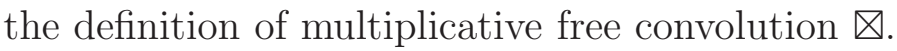

Let $\mathbb{C}^{+}\left(\mathbb{C}^{-}\right)$denote the open upper (lower) half of the complex plane. If $\mu \in \mathcal{M}$, then its Cauchy transform

$$
G_{\mu}(z)=\int_{-\infty}^{\infty} \frac{\mu(d t)}{z-t}, \quad z \in \mathbb{C}^{+} .
$$

Following Maassen [18] and Bercovici and Voiculescu 4], in the sequel we will consider reciprocal Cauchy transform

$$
F_{\mu}(z)=\frac{1}{G_{\mu}(z)}
$$

Let $\mathcal{F}$ denote the corresponding class of reciprocal Cauchy transforms of all $\mu \in$ $\mathcal{M}$. This class admits the simple description.

The class of all analytic functions $F: \mathbb{C}^{+} \rightarrow \mathbb{C}^{+} \cup \mathbb{R}$, say $\mathcal{N}$, is called Nevanlinna class.

The class $\mathcal{F} \subset \mathcal{N}$ of reciprocal Cauchy transforms of p-measures introduced above coincides with the subclass of Nevanlinna functions $F \in \mathcal{N}$ such that $F(z) / z \rightarrow 1$ as $z \rightarrow \infty$ nontangentially to $\infty$ (i.e., such that $|\operatorname{Re} z| / \operatorname{Im} z$ stays bounded). See Section 3 . 
This implies that $F_{\mu}$ has certain invertibility properties. To be precise, for two numbers $\alpha>0, \beta>0$ we set

$$
\Gamma_{\alpha}=\left\{z=x+i y \in \mathbb{C}^{+}:|x|<\alpha y\right\} \quad \text { and } \quad \Gamma_{\alpha, \beta}=\left\{z=x+i y \in \Gamma_{\alpha}: y>\beta\right\} .
$$

Then, by relation (3.4) of Section 3, for every $\alpha>0$ there exists $\beta=\beta(\mu, \alpha)$ such that $F_{\mu}$ has a left inverse $F_{\mu}^{(-1)}$ defined on $\Gamma_{\alpha, \beta}$. The function

$$
\varphi_{\mu}(z)=F_{\mu}^{(-1)}(z)-z
$$

is called the Voiculescu transform of $\mu$. It is not hard to show that $\operatorname{Im} \varphi_{\mu}(z) \leqslant 0$ for $z \in \Gamma_{\alpha, \beta}$ where $\varphi_{\mu}$ is defined. We also have $\varphi_{\mu}(z)=o(z)$ as $|z| \rightarrow \infty, z \in \Gamma_{\alpha}$.

If $\mu$ is the point measure $\delta_{a}$ at $a$, then $F(z)=z-a$ whereas $F(z)=z+i b, b \in \mathbb{R}$, corresponds to the Cauchy distribution with density $x \mapsto b /\left(\pi\left(x^{2}+b^{2}\right)\right)$ which has infinite variance.

\section{ADDITIVE FREE CONVOLUTION}

Let $\mu_{1}$ and $\mu_{2}$ be p-measures in $\mathcal{M}$ and let $F_{\mu_{1}}(z)$ and $F_{\mu_{2}}(z)$ denote their reciprocal Cauchy transforms respectively. We shall define the free convolution $\mu_{1} \boxplus \mu_{2}$, based on $F_{\mu_{1}}(z)$ and $F_{\mu_{2}}(z)$ using the following result.

Theorem 2.1. There exist unique functions $Z_{1}(z)$ and $Z_{2}(z)$ in the class $\mathcal{F}$ such that, for $z \in \mathbb{C}^{+}$,

$$
z=Z_{1}(z)+Z_{2}(z)-F_{\mu_{1}}\left(Z_{1}(z)\right) \quad \text { and } \quad F_{\mu_{1}}\left(Z_{1}(z)\right)=F_{\mu_{2}}\left(Z_{2}(z)\right) .
$$

The function $F_{\mu_{1}}\left(Z_{1}(z)\right)$ is in $\mathcal{F}$ again, hence there exists some p-measure $\mu$ such that $F_{\mu_{1}}\left(Z_{1}(z)\right)=F_{\mu}(z)$, where $F_{\mu}(z)=1 / G_{\mu}(z)$ and $G_{\mu}(z)$ denotes the Cauchy transform (2.1) of $\mu$.

Since the p-measure $\mu$ depends on $\mu_{1}$ and $\mu_{2}$ only, we define $\mu_{1} \boxplus \mu_{2}:=\mu$. The symmetry of relation (2.3) obviously implies that this operation is commutative and associative. Furthermore, choosing $\mu_{2}=\delta_{a}$ in (2.3), where $\delta_{a}$ denotes a Dirac measure concentrated at the point $a$, we get $\mu_{1} \boxplus \delta_{a}=\mu_{1} * \delta_{a}$. Definition (2.3) does not restrict the class of p-measures and allows an obvious extension to the case of multiplicative convolutions, described below.

Moreover, on any set $\Gamma_{\alpha, \beta}$, where both functions $\varphi_{\mu_{1}}(z)$ and $\varphi_{\mu_{2}}(z)$ are defined, we obtain

$$
\varphi_{\mu_{1} \boxplus \mu_{2}}(z)=\varphi_{\mu_{1}}(z)+\varphi_{\mu_{2}}(z) .
$$

The equation (2.4) for the distribution $\mu_{1} \boxplus \mu_{2}$ of $X+Y$, where $X$ and $Y$ are free random variables is due to Voiculescu [27. He considered p-measures $\mu$ with compact support. The result was extended by Maassen 18 to p-measures with finite variance; the general case was proved by Bercovici and Voiculescu [4. Note here that Voiculescu's and Bercovici's approach to the definition of $\mu_{1} \boxplus \mu_{2}$ based on the operator algebras. Maassen's analytic approach to the definition is closer to the one presented here. 
We see from (2.4) that our definition of $\mu_{1} \boxplus \mu_{2}$ coincides with the Voiculescu, Bercovici, Maassen definition. Pastur and Vasilchuk [23] (2000) studied the normalized eigenvalue counting measure of the sum of two $n \times n$ unitary matrices rotated independently by random unitary Haar distributed measures. They established the convergence in probability as $n \rightarrow \infty$ to a limiting nonrandom measure. They derive functional equations for the Cauchy transforms of limiting distributions assuming the existence of the mean of the limiting measures. It follows from Theorem 2.1 that their equations are equivalent to (2.3).

Using Speicher's combinatorial approach [25 (1998) to freeness, Biane [8] (1998) showed that there exists a unique function $F \in \mathcal{F}$ such that $G_{\mu_{1} \boxplus \mu_{2}}(z)=$ $G_{\mu_{1}}(F(z))$ for all $z \in \mathbb{C}^{+}$. It follows from Theorem 2.1 that $F(z)$ in Biane's assertion is $Z_{1}(z)$ in (2.3).

Corollary 2.2. Let $\mu_{1}, \ldots, \mu_{n} \in \mathcal{M}$. There exist unique functions $Z_{1}(z), \ldots, Z_{n}(z)$ in the class $\mathcal{F}$ such that, for $z \in \mathbb{C}^{+}$,

$z=Z_{1}(z)+\cdots+Z_{n}(z)-(n-1) F_{\mu_{1}}\left(Z_{1}(z)\right), \quad$ and $\quad F_{\mu_{1}}\left(Z_{1}(z)\right)=\cdots=F_{\mu_{n}}\left(Z_{n}(z)\right)$.

Moreover, $F_{\mu_{1} \boxplus \cdots \boxplus \mu_{n}}(z)=F_{\mu_{1}}\left(Z_{1}(z)\right)$ for all $z \in \mathbb{C}^{+}$.

Specializing to $\mu_{1}=\mu_{2}=\cdots=\mu_{n}=\mu$ denote $\mu_{1} \boxplus \cdots \boxplus \mu_{n}=\mu^{n \boxplus}$. Then we get

Corollary 2.3. Let $\mu \in \mathcal{M}$. There exists an unique function $Z \in \mathcal{F}$ such that

$$
z=n Z(z)-(n-1) F_{\mu}(Z(z)), \quad z \in \mathbb{C}^{+},
$$

and $F_{\mu^{n \boxplus}}(z)=F_{\mu}(Z(z)), z \in \mathbb{C}^{+}$.

By (2.5), we see that $\left(Z^{(-1)}(z)-z\right) /(n-1)=z-F_{\mu}(z)$ for $z$ from some domain $\Gamma_{\alpha, \beta}$. It follows from this that $Z^{(-1)}(z)-z$ has an analytic continuation to $\mathbb{C}^{+}$ with values in $\mathbb{C}^{-} \cup \mathbb{R}$. Since $Z \in \mathcal{F}$, it is easy to see, that $\left(Z^{(-1)}(i y)-i y\right) / y \rightarrow 0$ as $y \rightarrow+\infty$. Hence $Z(z)=F_{\nu}(z), z \in \mathbb{C}^{+}$, where $\nu \in \mathcal{M}$ is infinitely divisible relative to the free additive convolution (the definition and the characterization of the $\boxplus$-infinitely divisibility see in this section below). Note that relation (2.5) holds if the integers $n$ are replaced by real numbers $t \geqslant 1$. This shows that there is a semigroup $\nu_{t} \in \mathcal{M}, t \geqslant 1$, such that $t \varphi_{\nu}=\varphi_{\nu_{t}}$.

This fact was shown by Biane 8], using operator algebraic arguments.

Having defined the Voiculescu semigroup $(\mathcal{M}, \boxplus)$, basing on the properties of the functions of the subclass $\mathcal{F}$ of the class $\mathcal{N}$ of Nevanlinna functions, we shall proceed by studying multiplicative free convolutions. 


\section{Multiplicative free CONVOLution ON $\mathbb{R}_{+}$}

Let $\mathcal{M}_{+}$be the set of p-measures $\mu$ on $\mathbb{R}_{+}=[0,+\infty)$ such that $\mu(\{0\})<1$. Define, following Voiculescu [28], the $\psi_{\mu}$-function of a p-measure $\mu \in \mathcal{M}_{+}$, by

$$
\psi_{\mu}(z)=\int_{\mathbb{R}_{+}} \frac{z \xi}{1-z \xi} \mu(d \xi)
$$

for $z \in \mathbb{C} \backslash \mathbb{R}_{+}$. The measure $\mu$ is completely determined by $\psi_{\mu}$ because $z\left(\psi_{\mu}(z)+\right.$ $1)=G_{\mu}(1 / z)$. Note that $\psi_{\mu}: \mathbb{C} \backslash \mathbb{R}_{+} \rightarrow \mathbb{C}$ is an analytic function such that $\psi_{\mu}(\bar{z})=\overline{\psi_{\mu}(z)}$, and $z\left(\psi_{\mu}(z)+1\right) \in \mathbb{C}^{+}$for $z \in \mathbb{C}^{+}$. Consider the function

$$
R_{\mu}(z):=\psi_{\mu}(z) /\left(1+\psi_{\mu}(z)\right), \quad z \in \mathbb{C} \backslash \mathbb{R}_{+} .
$$

It is easy to see that $R_{\mu}(z) \in \mathcal{N}$ and $R_{\mu}(z)$ is analytic and nonpositive on the negative real axis $(-\infty, 0)$. In addition, for $x>0, R_{\mu}(-x) \rightarrow 0$ as $x \rightarrow 0$.

Denote by $\mathcal{K}$ the subclass of $\mathcal{N}$ of functions $f$ such that $f(z)$ is analytic and nonpositive on the negative real axis, and, for $x>0, f(-x) \rightarrow 0$ as $x \rightarrow 0$.

By Krein's results (see Section 3), the function $R_{\mu}(z)$, being analytic and nonpositive on $(-\infty, 0)$, and $\lim _{x \rightarrow 0, x>0} R_{\mu}(-x)=0$, belongs to $\mathcal{K}$ and admits by Corollary $3.3(2)$ (like all functions in $\mathcal{K}$ ) the following representation

$$
\frac{R_{\mu}(z)}{z}=a+\int_{(0, \infty)} \frac{\tau(d t)}{t-z}, \quad 0<\arg z<2 \pi
$$

where $a \geqslant 0$ and $\tau$ is a nonnegative measure such that

$$
\int_{(0, \infty)} \frac{\tau(d t)}{1+t}<\infty
$$

In view of Proposition 3.4, see Section 3, the function $R_{\mu}$ has the inverse function $\tilde{\chi}_{\mu}$ on the image $R_{\mu}\left(i \mathbb{C}^{+}\right)$. We define the $\Sigma$-transform of $\mu$ as the function

$$
\Sigma_{\mu}(z):=\tilde{\chi}_{\mu}(z) / z, \quad z \in R_{\mu}\left(i \mathbb{C}^{+}\right) .
$$

Note that $R_{\mu}\left(i \mathbb{C}^{+}\right) \supseteq \Gamma_{\alpha, \beta, \Delta}^{+}:=\left\{z \in \mathbb{C}^{+}: \beta<|z|<\Delta, \alpha<\arg z<2 \pi-\alpha\right\}$ for some $0<\beta<\Delta$ and $\alpha \in(0, \pi)$. In addition we conclude from (2.8) that $\arg R_{\mu}(z) \geqslant \arg z$ for $z \in \mathbb{C}^{+}$and $\arg R_{\mu}(z)=\pi$ for $z \in(-\infty, 0)$. Therefore $\arg \Sigma_{\mu}(z) \leqslant 0, \operatorname{Im} z \geqslant 0$, and $\arg \Sigma_{\mu}(z)=0, z \in(-\infty, 0)$, where $\Sigma_{\mu}(z)$ is defined.

Let $\mu_{1}$ and $\mu_{2}$ denote $p$-measures in $\mathcal{M}_{+}$with corresponding transforms $R_{\mu_{1}}$ and $R_{\mu_{2}}$ defined in (2.7), which are in the klass $\mathcal{K}$.

We shall define the free multiplicative convolution using the transforms $R_{\mu_{1}}$ and $R_{\mu_{2}}$ by means of the following characterization which (after exchanging addition with multiplication) is identical to characterization (2.3) for the additive convolution. 
Theorem 2.4. There exist two uniquely determined functions $Z_{1}(z)$ and $Z_{2}(z)$ in the Krein class $\mathcal{K}$ such that

$$
Z_{1}(z) Z_{2}(z)=z R_{\mu_{1}}\left(Z_{1}(z)\right) \quad \text { and } \quad R_{\mu_{1}}\left(Z_{1}(z)\right)=R_{\mu_{2}}\left(Z_{2}(z)\right), \quad z \in \mathbb{C}^{+} .
$$

Introduce

$$
R(z):=R_{\mu_{1}}\left(Z_{1}(z)\right) \quad \text { and } \quad \psi(z):=R(z) /(1-R(z)), \quad z \in \mathbb{C}^{+} .
$$

Then, by (2.8) for $R_{\mu_{1}}$ and $Z_{1}$, we note that $R(z)$ and $R(z) / z$ are functions in the class $\mathcal{N}$ and $R(-x) \rightarrow 0$ as $x \rightarrow 0$ for $x>0$. Hence, by Corollary 3.3 $(1), R(z) \in \mathcal{K}$. Using this assertion we easily see that $\psi(z) \in \mathcal{N}, \psi(z) / z \in \mathcal{N}$, and $\lim _{x \rightarrow 0, x>0} \psi(-x)=0$. Moreover, by representation (2.8) for $R(z)$, we have $\lim _{x \rightarrow-\infty} \psi(x) / x=0$. Hence the function $\psi(z) / z$ admits the representation (2.8) with $a=0$, i.e.,

$$
\frac{\psi(z)}{z}=\int_{(0, \infty)} \frac{\tau_{\psi}(d t)}{t-z}=\int_{(0, \infty)} \frac{u}{1-u z} \mu_{\psi}(d u), \quad z \in \mathbb{C}^{+},
$$

where $\tau_{\psi}$ is a nonnegative measure satisfying condition (2.9) and $\mu_{\psi}$ is a nonnegative finite measure.

Note that $\lim _{x \rightarrow-\infty} \psi(x)=-1$ if and only if in representation (2.8) for $R(z)$ either $a>0$ or $\tau((0, \infty))=\infty$. In this case, by (2.11), we may represent $\psi(z) \in \mathcal{K}$ as $\psi=\psi_{\mu}$, see (2.6), with some p-measure $\mu \in \mathcal{M}_{+}$such that $\mu(\{0\})=0$. In addition $R \in \mathcal{K}$ may be represented as $R(z)=R_{\mu}(z), z \in \mathbb{C}^{+}$. Therefore $\psi_{\mu_{1}}\left(Z_{1}(z)\right)=\psi_{\mu}(z)$.

Let in (2.8) for $R(z) a=0$ and $\tau((0, \infty))<\infty$. Then, as it is easy to see, $\lim _{x \rightarrow-\infty} \psi(x)=-p=-\tau((0, \infty)) /(1+\tau((0, \infty)))$. Again, by (2.11), we get for $\psi(z)$ representation (2.6] with some p-measure $\mu \in \mathcal{M}_{+}$and $\mu(\{0\})=1-p$. Thus, $\psi(z)=\psi_{\mu}(z)$ and $R(z)=R_{\mu}(z), z \in \mathbb{C}^{+}$. Hence $\psi_{\mu_{1}}\left(Z_{1}(z)\right)=\psi_{\mu}(z)$.

The p-measure $\mu$ is determined uniquely by the p-measures $\mu_{1}$ and $\mu_{2}$.

We define $\mu:=\mu_{1} \otimes \mu_{2}$. Since $R_{\mu_{1}}\left(Z_{1}(z)\right)=R_{\mu_{2}}\left(Z_{2}(z)\right)$ for $z \in \mathbb{C}^{+}$, we have $\mu_{1} \otimes \mu_{2}=\mu_{2} \otimes \mu_{1}$ and it is easily verified that this convolution is associative as well.

From Theorem 2.4 we conclude that the relation $\Sigma_{\mu_{1}}(z) \Sigma_{\mu_{2}}(z)=\Sigma_{\mu}(z)$ holds on $R_{\mu_{1}}\left(i \mathbb{C}^{+}\right) \cap R_{\mu_{2}}\left(i \mathbb{C}^{+}\right)$. This relation is due to Voiculescu [28] and Bercovici and Voiculescu 4 .

Corollary 2.5. Let $\mu_{1}, \ldots, \mu_{n} \in \mathcal{M}_{+}$. There exist unique functions $Z_{1}(z), \ldots, Z_{n}(z)$ in the class $\mathcal{K}$ such that, for $z \in \mathbb{C}^{+}$,

$$
Z_{1}(z) \ldots Z_{n}(z)=z\left(R_{\mu_{1}}\left(Z_{1}(z)\right)\right)^{n-1}, \quad \text { and } \quad R_{\mu_{1}}\left(Z_{1}(z)\right)=\cdots=R_{\mu_{n}}\left(Z_{n}(z)\right) .
$$

Moreover, $R_{\mu_{1} \otimes \cdots \boxplus \mu_{n}}(z)=R_{\mu_{1}}\left(Z_{1}(z)\right)$ for all $z \in \mathbb{C}^{+}$.

Let $\mu_{1}=\mu_{1}=\cdots=\mu_{n}=\mu$. Denote $\mu_{1} \otimes \cdots \otimes \mu_{n}=\mu^{n \otimes}$. 
Corollary 2.6. Let $\mu \in \mathcal{M}_{+}$. There exists an unique function $Z \in \mathcal{K}$ such that

$$
(Z(z))^{n}=z\left(R_{\mu}(Z(z))\right)^{n-1}, \quad z \in \mathbb{C}^{+},
$$

and $R_{\mu^{n \otimes}}(z)=R_{\mu}(Z(z)), z \in \mathbb{C}^{+}$.

Again relation (2.12) holds if we replace integers $n$ by real numbers $t \geqslant 1$. This shows that there is a semigroup $\nu_{t} \in \mathcal{M}_{+}, t \geqslant 1$, such that $\left(\Sigma_{\nu}(z)\right)^{t}=\Sigma_{\nu_{t}}(z)$.

Thus we have defined the Voiculescu semigroup $\left(\mathcal{M}_{+}, \nabla\right)$, based on properties of functions of the Krein subclass $\mathcal{K}$ of the class $\mathcal{N}$ of Nevanlinna functions. In the following we consider the case of spectral p-measures on the unit circle $\mathbb{T}$.

\section{Multiplicative FREE CONVOLUtion ON THE UNit CIRCLE}

Let $\mu$ be a p-measure on $\mathbb{T}$. Following Voiculescu 28, we define a transform of the p-measure $\mu$ on $\mathbb{T}$, by

$$
\psi_{\mu}(z)=\int_{\mathbb{T}} \frac{z \xi}{1-z \xi} \mu(d \xi) .
$$

The function $\psi$ has a convergent power series representation in $\mathbb{D}:=\{z \in \mathbb{C}$ : $|z|<1\}$, the open unit disk of $\mathbb{C}$, such that $\psi_{\mu}(0)=0$.

Let $\mathcal{M}_{*}$ denote the set of p-measures on $\mathbb{T}$ such that $\int_{\mathbb{T}} \xi \mu(d \xi) \neq 0$.

If $\mu \in \mathcal{M}_{*}$, it follows that the function

$$
Q_{\mu}:=\psi_{\mu} /\left(1+\psi_{\mu}\right)
$$

has a right inverse $Q_{\mu}^{(-1)}$, defined in a neighborhood of 0 denoted by $\mathbb{D}_{\alpha}:=\{z \in$ $\mathbb{C}:|z|<\alpha\}$ with some $0<\alpha \leqslant 1$, such that $Q_{\mu}^{(-1)}(0)=0$. Let

$$
\Sigma_{\mu}(z)=Q_{\mu}^{(-1)}(z) / z
$$

denote the so called $\Sigma$-transform of $\mu$.

Denote by $\mathcal{C}$ the class of analytic functions $F(z)$ on $\mathbb{D} \rightarrow-i\left(\mathbb{C}^{+} \cup \mathbb{R}\right)$ introduced by Carathéodory.

Note that

$$
Q_{\mu}(z)=\frac{\psi_{\mu}(z)}{1+\psi_{\mu}(z)}=\frac{F(z)-1}{F(z)+1}
$$

where $F(z):=1+2 \psi_{\mu}(z)$ is a function of Carathéodory's class $\mathcal{C}$. Such functions $F(z)$, by (3.1) and $F(0)=1$, see Section 3, have the form

$$
F(z)=\int_{\mathbb{T}} \frac{\xi+z}{\xi-z} \sigma(d \xi),
$$

where $\sigma$ is a p-measure.

Define $\mathcal{S}$ to be so called Schur class of analytic functions $\mathbb{D} \rightarrow \overline{\mathbb{D}}$ (see Section 3), where $\overline{\mathbb{D}}$ is the closure of $\mathbb{D}$. 
We see from (2.13), that $Q_{\mu} \in \mathcal{S}$ and since $\psi_{\mu}(0)=0$ and $\mu \in \mathcal{M}_{*}, Q_{\mu}(0)=$ $0, Q_{\mu}^{\prime}(0) \neq 0$.

In the sequel we denote by $\mathcal{S}_{*}$ the subclass of $\mathcal{S}$ which consists of Schur functions $Q$ with properties $Q(0)=0$ and $Q^{\prime}(0) \neq 0$.

Since $Q_{\mu} \in \mathcal{S}_{*}$, by the Schwarz lemma, we have $\left|Q_{\mu}(z) / z\right| \leqslant 1$ for $z \in \mathbb{D}$. Hence $\left|Q_{\mu}^{(-1)}(z) / z\right| \geqslant 1$ in a neighborhood of 0 . Moreover, both (3.2) induces a one-toone correspondence between the classes $\mathcal{C}$ and $\mathcal{S}$, and (2.13) induces a one-to-one correspondence between functions $F \in \mathcal{C}$ such that $F(0)=1$ and $F^{\prime}(0) \neq 0$, and functions $Q_{\mu}$ of the class $\mathcal{S}_{*}$.

Let $\mu_{1}$ and $\mu_{2}$ denote p-measures in $\mathcal{M}_{*}$ and let $Q_{\mu_{1}}$ and $Q_{\mu_{2}}$ be Schur functions which correspond to these measures, by (2.13). We now define the free multiplicative free convolution $\mu_{1} \otimes \mu_{2}$ based on $Q_{\mu_{1}}$ and $Q_{\mu_{2}}$ using the following characterization.

Theorem 2.7. There exist two functions $Z_{1}(z)$ and $Z_{2}(z)$ in the set $\mathcal{S}_{*}$ such that

$$
Z_{1}(z) Z_{2}(z)=z Q_{\mu_{1}}\left(Z_{1}(z)\right) \quad \text { and } \quad Q_{\mu_{1}}\left(Z_{1}(z)\right)=Q_{\mu_{2}}\left(Z_{2}(z)\right) .
$$

The functions $Z_{1}(z)$ and $Z_{2}(z)$ are unique solutions of 2.14 in the class $\mathcal{S}_{*}$.

Consider the function $Q_{\mu_{1}}\left(Z_{1}(z)\right)$. It is easy to see that it belongs to Schur's class $\mathcal{S}$ and $Q_{\mu_{1}}\left(Z_{1}(0)\right)=0, Q_{\mu_{1}}^{\prime}(0) Z_{1}^{\prime}(0) \neq 0$. Therefore $Q_{\mu_{1}}\left(Z_{1}\right) \in \mathcal{S}_{*}$ and $Q_{\mu_{1}}\left(Z_{1}(z)\right)=Q_{\mu}(z)$ for $z \in \mathbb{D}$, where $Q_{\mu}(z)$ has form (2.13) for some p-measure $\mu \in \mathcal{M}_{*}$. This measure is determined uniquely by the p-measures $\mu_{1}$ and $\mu_{2}$. Define $\mu:=\mu_{1} \otimes \mu_{2}$. Since $Q_{\mu_{1}}\left(Z_{1}(z)\right)=Q_{\mu_{2}}\left(Z_{2}(z)\right)$ for $z \in \mathbb{D}$, we get $\mu_{1} \otimes \mu_{2}=$ $\mu_{2} \otimes \mu_{1}$. It is easy to verify that the operation $\nabla$ is associative. Using relation (2.13) between the function $Q_{\mu}(z) \in \mathcal{S}_{*}$ and the function $\psi_{\mu}(z)$, we conclude that $\psi_{\mu}(z)=\psi_{\mu_{1}}\left(Z_{1}(z)\right)$ for $z \in \mathbb{D}$. In addition we have in some neighborhood of 0

$$
\frac{Q_{\mu_{1}}^{(-1)}(z)}{z} \frac{Q_{\mu_{2}}^{(-1)}(z)}{z}=\frac{Q_{\mu}^{(-1)}(z)}{z} \text { or } \quad \Sigma_{\mu_{1}}(z) \Sigma_{\mu_{2}}(z)=\Sigma_{\mu}(z) .
$$

This formula is due to Voiculescu [28, 3] (1992).

Note that Vasilchuk [26] (2001) studied the normalized eigenvalue counting measure of the product of two $n \times n$ unitary matrices and the measure of product of three $n \times n$ Hermitian positive matrices rotated independently by random unitary Haar distributed measures. He extended the method used in Pastur and Vasilchuk 23] and established the convergence in probability as $n \rightarrow \infty$ to a limiting nonrandom measure. Furthermore, he derived functional equations for the Herglotz and Cauchy transforms of limiting distributions under some restriction on counting measures. From Theorem 2.4 and Theorem 2.7 it follows that his equations are equivalent to (2.10) and (2.14).

For the multiplicative free convolution the analogues of Corrolary 2.2 and Corollary 2.3 hold. 
Corollary 2.8. Let $\mu_{1}, \ldots, \mu_{n} \in \mathcal{M}_{*}$. There exist uniquely determined functions $Z_{1}(z), \ldots, Z_{n}(z)$ in the class $\mathcal{S}_{*}$ such that, for $z \in \mathbb{D}$,

$$
\begin{aligned}
& Z_{1}(z) \ldots Z_{n}(z)=z\left(Q_{\mu_{1}}\left(Z_{1}(z)\right)\right)^{n-1}, \quad \text { and } Q_{\mu_{1}}\left(Z_{1}(z)\right)=\cdots=Q_{\mu_{n}}\left(Z_{n}(z)\right) \text {. } \\
& \text { Moreover, } Q_{\mu_{1} \bowtie \cdots \boxplus \mu_{n}}(z)=Q_{\mu_{1}}\left(Z_{1}(z)\right) \text { for all } z \in \mathbb{D} \text {. }
\end{aligned}
$$

Corollary 2.9. Let $\mu \in \mathcal{M}_{*}$. There exists an unique function $Z \in \mathcal{S}_{*}$ such that

$$
(Z(z))^{n}=z\left(Q_{\mu}(Z(z))\right)^{n-1}, \quad z \in \mathbb{D},
$$

and $Q_{\mu^{n \bowtie}}(z)=Q_{\mu}(Z(z)), z \in \mathbb{D}$.

Note that relation (2.16) holds if we replace integers $n$ by real $t \geqslant 1$. This shows that there is a semigroup $\nu_{t} \in \mathcal{M}_{*}, t \geqslant 1$ such that $\left(\Sigma_{\nu}(z)\right)^{t}=\Sigma_{\nu_{t}}(z)$.

Thus we have defined the Voiculescu semigroup $\left(\mathcal{M}_{*}, \otimes\right)$, based on properties of functions of the subclass $\mathcal{S}_{*}$ of the class $\mathcal{S}$ of Schur functions.

\section{ARIthmetic of P-MeAsures in Voiculescu's SEMigroups}

Now we consider the problem of the decomposition of measures $\mu$ of the commutative semigroups $(\mathcal{M}, \boxplus),\left(\mathcal{M}_{+}, \otimes\right)$, and $\left(\mathcal{M}_{*}, \nabla\right)$. In the sequel we shall denote these semigroups by a symbol $(\mathbf{M}, \circ)$, where $\mathbf{M}$ means $\mathcal{M}, \mathcal{M}_{+}, \mathcal{M}_{*}$ and $\circ$ means the operations $\boxplus, \otimes$. The following notions are analogues of the classical ones for $(\mathcal{M}, *)$.

We shall say that $\mu_{1} \in \mathbf{M}$ is a free factor or just factor of $\mu \in \mathbf{M}$ if there exists $\mu_{2} \in \mathbf{M}$ such that $\mu=\mu_{1} \circ \mu_{2}$. Every $\mu \in \mathbf{M}$ has factors. Indeed, we have $\mu=\delta_{a} \circ\left(\mu \circ \delta_{b}\right)$, where $b=-a, a \in \mathbb{R}$, in the case of the $\operatorname{semigroup}(\mathcal{M}, \boxplus)$, $b=1 / a, a>0$, in the case of $\left(\mathcal{M}_{+}, \nabla\right)$, and $b=1 / a, a \in \mathbb{T}$, in the case of $\left(\mathcal{M}_{*}, \otimes\right)$. Hence $\delta_{a}$ and $\mu \circ \delta_{b}$ are factors of $\mu$. Such factors are called improper. A p-measure $\mu$ which is not a Dirac measure is called indecomposable if it has improper factors only. Such p-measures may be regarded as simple elements of this semigroup. If $\mu$ in $(\mathbf{M}, \circ)$ is not indecomposable it is called decomposable. Two measures $\mu_{1}$ and $\mu_{2}$ are called equivalent, $\mu_{1} \sim \mu_{2}$, if $\mu_{1}=\mu_{2} \circ \delta_{a}$, where $a \in \mathbb{R}$ in the case of $(\mathcal{M}, \boxplus), a>0$ in the case of $\left(\mathcal{M}_{+}, \otimes\right)$, and $a \in \mathbb{T}$ in the case of $\left(\mathcal{M}_{*}, \otimes\right)$.

As in the classical theory of convolutions, a measure $\mu$ is called o-infinitely divisible (or i.d. for short) if, for every natural number $n, \mu$ can be written as $\mu=\nu_{n} \circ \nu_{n} \circ \cdots \circ \nu_{n}$ ( $n$ times) with $\nu_{n} \in \mathbf{M}$. The measure $\delta_{a}$ is necessarily infinitely divisible. Note that all i.d. measures are decomposable and Dirac measures have this property. As mentioned in the introduction a measure $\mu \in \mathbf{M}$ is to the class $I_{0}$ relative $\circ$ if $\mu$ is o-infinitely divisible and has no o-indecomposable factors.

Khintchine 15] (1937) was the first who studied the arithmetic of the semigroup $(\mathcal{M}, *)$ of distribution functions on $\mathbb{R}$ with respect to the convolution $*$. He derived for this semigroup the three basic results.

1. Limit of triangular arrays. Obviously an i.d. element $\mu$ of $(\mathcal{M}, *)$ can always be represented as the limit of a convergent infinitesimal triangular array. 
2. Classification. Any element $\mu$ of $(\mathcal{M}, *)$ belongs to one of the following classes. Either

(1) $\mu$ is indecomposable,

(2) $\mu$ is decomposable (possibly i.d.) and has an indecomposable factor,

(3) $\mu$ is i.d. and has no indecomposable factors. (This class of p-measures is denoted by $I_{0}$.)

(Compare the description of Delphic semigroups in the introduction.)

3. Representation. For each $\mu$ in $\mathcal{M}$ one may decompose

$$
\mu=\nu * \mu_{1} * \mu_{2} * \ldots
$$

in at least one way, where $\nu$ is i.d. and has no indecomposable sub-factor, and each $\mu_{j}$ is indecomposable. The convolution product is at most countable and may be finite or void.

Note that Gaussian distributions (Cramér (1936)), Poisson distributions (Raikov (1937)) and the convolution of Gaussian and Poisson distributions (Linnik (1957)) belong to the class $I_{0}$. Hence in the $\operatorname{semigroup}(\mathcal{M}, *)$ the class $I_{0}$ has nontrivial elements (besides the trivial units $\delta_{a}$ ).

A number of papers have been devoted to the study of the arithmetic of semigroups of p-measures. We refer the reader to the monograph of Linnik and Ostrovskii [16] (1977), the surveys of Livshic, Ostrovskii and Chistyakov [17] (1975), Ostrovskii [21] (1977), 22] (1986).

We shall consider the semigroups ( $M, \circ)$ introduced above and we study their arithmetic using the theory of Delphic semigroups, (see Kendall [14, Davidson [9] -11]). Kendall and Davidson developed Khintchine's theory for a wide class of semigroups (Delphic) where Khintchine's basic theorems remain valid.

In the semigroup $(\mathcal{M}, \boxplus)$ i.d. p-measures were first considered in Voiculescu [27], where compactly supported $\boxplus$-i.d. measures were characterized. P-measures with a finite variance were considered in Maassen [18] and Bercovichi and Voiculescu 4 ] gave characterization of general i.d. p-measures $\mu \in \mathcal{M}$. There is an analogue of the Lévy-Khintchine formula, (see [29], 3], 4]) which states that a p-measure $\mu$, on $\mathbb{R}$, is i.d. if and only if the function $\varphi_{\mu}(z)$ has an analytic continuation to the whole of $\mathbb{C}^{+}$, with values in $\mathbb{C}^{-} \cup \mathbb{R}$, and one has

$$
\lim _{y \rightarrow+\infty} \frac{\varphi_{\mu}(i y)}{y}=0 .
$$

By the Nevanlinna representation for such function, we know that there exist a real number $\alpha$, and a finite nonnegative measure $\nu$, on $\mathbb{R}$, such that

$$
\varphi_{\mu}(z)=\alpha+\int_{\mathbb{R}} \frac{1+u z}{z-u} \nu(d u), \quad z \in \mathbb{C}^{+} .
$$

There is a one-to-one correspondence between functions $\varphi_{\mu}(z)$ and couples $(\alpha, \nu)$. For this reason we shall sometimes write $\varphi_{\mu}=(\alpha, \nu)$. 
Formula (2.18) is an analogue of the well-known Lévy-Khintchine formula for the logarithm of characteristic functions $\varphi(t ; \mu):=\int_{\mathbb{R}} e^{i t u} \mu(d u), t \in \mathbb{R}$, of $*$-i.d. measures $\mu \in \mathcal{M}$. A measure $\mu \in \mathcal{M}$ is $*$-i.d. if and only if there exist a finite nonnegative Borel measure $\nu$ on $\mathbb{R}$, and a real number $\alpha$ such that

$$
\log \varphi(t ; \mu)=f_{\mu}(t):=\exp \left\{i \alpha t+\int_{\mathbb{R}}\left(e^{i t u}-1-\frac{i t u}{1+u^{2}}\right) \frac{1+u^{2}}{u^{2}} \nu(d u)\right\}, \quad t \in \mathbb{R},
$$

where $\left(e^{i t u}-1-i t u /\left(1+u^{2}\right)\right)\left(1+u^{2}\right) / u^{2}$ will be interpreted as $-t^{2} / 2$ for $u=0$. There is a one-to-one correspondence between functions $f_{\mu}(t)$ and couples $(\alpha, \nu)$. For this reason we shall sometimes write $f_{\mu}=(\alpha, \nu)$.

In the classical case the precise formulation of the Khintchine limit theorem for $(\mathcal{M}, *)$ is as follows:

Let $\left\{\mu_{n k}: n \geqslant 1,1 \leqslant k \leqslant n\right\}$ be an array of infinitesimal measures in $\mathcal{M}$, i.e.,

$$
\lim _{n \rightarrow \infty} \max _{1 \leqslant k \leqslant n} \mu_{n k}(\{u:|u|>\varepsilon\})=0
$$

for every $\varepsilon>0$. In order that $\mu \in \mathcal{M}$ be the limit in the weak topology of distributions $\mu^{(n)}=\delta_{a_{n}} * \mu_{n 1} * \mu_{n 2} * \cdots * \mu_{n n} \rightarrow \mu$ for some suitably chosen constants $a_{n}$, it is necessary and sufficient that $\mu$ be i.d.

(Without loss of generality, we shall in the sequel consider arrays of the length $n$ instead of $k_{n}$ ).

The Khintchine limit theorem in free probability theory has the same form for the measures $\mu^{(n)}=\delta_{a_{n}} \boxplus \mu_{n 1} \boxplus \mu_{n 2} \boxplus \cdots \boxplus \mu_{n n}$.

This theorem was early proved by Bercovici and Pata [7] (2000). We give another proof of this result as a consequence of the theory of Delphic semigroups.

The i.d. measures in $\left(\mathcal{M}_{+}, \otimes\right)$ have been characterized by Voiculescu [28, Bercovici and Voiculescu [3, 4]. There is an analogue of the Lévy-Khintchine formula which states that a measure $\mu \in \mathcal{M}_{+}$is $\otimes$-i.d. if and only if there exist a finite nonnegative measure $\nu$ on $(0, \infty)$ and real numbers $a$ and $b \geqslant 0$ such that

$$
\Sigma_{\mu}(z)=\exp \left\{a-b z+\int_{\mathbb{R}_{+}} \frac{1+u z}{z-u} \nu(d u)\right\}, \quad 0<\arg z<2 \pi .
$$

For this reason we will write $\Sigma_{\mu}=(a, b, \nu)$.

In other words, a measure $\mu \in \mathcal{M}_{+}$is $\bigotimes$-i.d. if and only if

$$
\Sigma_{\mu}(z)=\exp \{-u(z)\}, \quad z \in \mathbb{C}^{+},
$$

where $u(z) \in \mathcal{N}$ and $u(z)$ is analytic and real valued on the negative half-line $(-\infty, 0)$.

Khintchine's limit problem for multiplicative free convolution may be formulated as follows. 
Let $\left\{\mu_{n k}: n \geqslant 1,1 \leqslant k \leqslant n\right\}$ be an array of measures in $\mathcal{M}_{+}$such that

$$
\lim _{n \rightarrow \infty} \max _{1 \leqslant k \leqslant n} \mu_{n k}(\{u:|u-1|>\varepsilon\})=0
$$

for every $\varepsilon>0$. The measures $\mu_{n k} \in \mathcal{M}_{+}$are called infinitesimal.

We shall characterize in Theorem 2.10 the class of p-measures $\mu \in \mathcal{M}_{+}$such that $\mu^{(n)}=\delta_{a_{n}} \otimes \mu_{n 1} \otimes \mu_{n 2} \cdots \otimes \mu_{n n} \rightarrow \mu$ in the weak topology for some suitably chosen positive constants $a_{n}$.

The i.d. measures of the semigroup $\left(\mathcal{M}_{*}, \nabla\right)$ were characterized in [28], 3]. There is an analogue of the Lévy-Khintchine formula which states that a measure $\mu \in \mathcal{M}_{*}$ is $\nabla$-i.d. if and only if there exist a finite nonnegative measure $\nu$ on $\mathbb{T}$ and a real number $a$ such that

$$
\Sigma_{\mu}(z)=\exp \left\{i a+\int_{\mathbb{T}} \frac{1+z \xi}{1-z \xi} \nu(d \xi)\right\}, \quad z \in \mathbb{D} .
$$

For this reason we will write $\Sigma=(a, \nu)$.

In other words, a measure $\mu \in \mathcal{M}_{*}$ is $\bigotimes$-i.d. if and only if

$$
\Sigma_{\mu}(z)=\exp \{v(z)\}, \quad z \in \mathbb{D},
$$

where $v(z) \in \mathcal{C}$.

Let $\left\{\mu_{n k}: n \geqslant 1,1 \leqslant k \leqslant n\right\}$ be an array of measures in $\mathcal{M}_{*}$. We shall call the measures $\mu_{n k}$ infinitesimal if

$$
\lim _{n \rightarrow \infty} \max _{1 \leqslant k \leqslant n} \mu_{n k}(\{\xi:|\arg \xi|>\varepsilon\})=0 .
$$

The Khintchine limit problem for multiplicative free convolution for measures $\mu \in \mathcal{M}_{*}$ has the same form as in the case of $\mu \in \mathcal{M}_{+}$with constants $a_{n} \in \mathbb{T}$.

We give the solution of the Khintchine limit problem, proving the following result.

Theorem 2.10. Let $\left\{\mu_{n k}: n \geqslant 1,1 \leqslant k \leqslant n\right\}$ be infinitesimal probability measures in the semigroup (M,o). The family of limit measures of sequences $\mu^{(n)}=\delta_{a_{n}} \circ \mu_{n 1} \circ \mu_{n 2} \circ \cdots \circ \mu_{n n}$ for some suitably chosen constants $a_{n}$ coincides with the family of o-infinitely divisible p-measures.

The arithmetic of the semigroups $(\mathbf{M}, \circ)$ is described in the following results.

Theorem 2.11. The element $\mu$ of $(\mathbf{M}, \circ)$ can be classified as follows. Either

(1) $\mu$ is indecomposable,

(2) $\mu$ is decomposable (possibly infinitely divisible) and has an indecomposable factor,

(3) $\mu$ is infinitely divisible and has no indecomposable factors. (This class will be denoted by $I_{0}$.)

p-Measures may be decomposed as follows 
Theorem 2.12. Every probability measure $\mu$, which has indecomposable factors, can be expressed in the form

$$
\mu=\mu_{0} \circ \mu_{1} \circ \mu_{2} \circ \ldots,
$$

where $\mu_{0} \in I_{0}$ and the probability measures $\mu_{1}, \mu_{2}, \ldots$ are indecomposable (its number may be finite or enumerable).

We will show that representation (2.27) is not unique.

The class $I_{0}$ in $(\mathbf{M}, \circ)$, mentioned above may be described as follows.

Theorem 2.13. In Voiculescu's semigroup $(\mathbf{M}, \circ)$ the class $I_{0}$ is trivial, that is $I_{0}$ is the class of Dirac measures.

Concerning indecomposable p-measures we show the following result.

Theorem 2.14. The probability measures with support consisting of a finite number of points are indecomposable in $(\mathbf{M}, \circ)$.

Corollary 2.15. The class of indecomposable elements of $(\mathbf{M}, \circ)$ is dense in $(\mathbf{M}, \circ)$ in the weak topology.

Theorem 2.11 describes the class $I_{0}$ as the class of i.d. elements of $(\mathbf{M}, \circ)$ which have i.d. components only. Bercovici and Voiculescu 5] proved that a semicircular measure does not belong to the class $I_{0}$ in the semigroup $(\mathcal{M}, \boxplus)$. This result follows from Theorem 2.13.

Speicher and Woroudi [24] (1997) introduced a further convolution operation on $\mathcal{M}$ denoted $\biguplus$. Let $\mu \in \mathcal{M}$. Denote as before by $G_{\mu}$ the Cauchy transform of $\mu$ and by $F_{\mu}=1 / G_{\mu}: \mathbb{C}^{+} \rightarrow \mathbb{C}^{+}$its reciprocal. We have $\operatorname{Im} z \leqslant \operatorname{Im} F_{\mu}(z)$ so that the function $E_{\mu}(z):=z-F_{\mu}(z)$ maps $\mathbb{C}^{+}$to $\mathbb{C}^{-} \cup \mathbb{R}$, and, in addition, $E_{\mu}(z) / z \rightarrow 0$ as $z \rightarrow \infty, z \in \Gamma_{\alpha}$ for any fixed $\alpha>0$. Conversely, if $E: \mathbb{C}^{+} \rightarrow \mathbb{C}^{-} \cup \mathbb{R}$ is an analytic function so that $E(z) / z \rightarrow 0$ as $z \rightarrow \infty, z \in \Gamma_{\alpha}$ for any fixed $\alpha$, then there exists $\mu \in \mathcal{M}$ such that $E_{\mu}=E$. This observation leads to the formal definition of the Boolean convolution. Given $\mu, \nu \in \mathcal{M}$, there exists $\rho \in \mathcal{M}$ such that

$$
E_{\rho}=E_{\mu}+E_{\nu} .
$$

The measure $\rho$ is called the Boolean convolution of $\mu$ and $\nu$, and it denoted $\mu \biguplus \nu$. Boolean convolution is an associative and commutative law, with $\delta_{0}$ as the zero element. We have $\delta_{s} \biguplus \delta_{t}=\delta_{s+t}$, but generally $\delta_{t} \biguplus \mu$ is not a translate of $\mu$. Speicher and Woroudi 24 treated the central and Poisson limit theorems, characterized i.d. and stable distributions and proved analogues of the classical theorems of Cramér, Marcinkiewicz, Kac and Loève. Bercovici and Pata 6] (1999) established limits law for Boolean convolutions.

Speicher and Woroudi 24 noted that all p-measures $\mu$ are i.d. It is easy to see that the class of indecomposable elements in the semigroup $(\mathcal{M}, \biguplus)$ is empty. Moreover, the class of i.d. elements coincides with the class $I_{0}$. Therefore Theorem 2.12 in $(\mathcal{M}, \biguplus)$ obviously holds. 


\section{Auxiliary Results}

In the following we state some results about classes of analytic functions (see Akhiezer [1 (1965), Section 3, and Akhiezer and Glazman 2] (1963), Section 6, $\S 59)$.

By $\mathcal{C}$ we denote C. Carathéodory's class of analytic functions $F(z): \mathbb{D} \rightarrow$ $\{z: \operatorname{Re} z \geqslant 0\}$. A function $F$ is in $\mathcal{C}$ if and only if it admits the following representation (Herglotz, G., Riesz, F.)

$$
F(z)=i a+\int_{\mathbb{T}} \frac{\xi+z}{\xi-z} \sigma(d \xi)
$$

where $a=\operatorname{Im} F(0), \mathbb{T}$ is the unit circle, and $\sigma$ is finite nonnegative measure. The number $a$ and the measure $\sigma$ are uniquely determined by $F$.

By $\mathcal{S}$ we denote J. Schur's class of analytic functions $\varphi(z): \mathbb{D} \rightarrow \overline{\mathbb{D}}$. The classes $\mathcal{C}$ and $\mathcal{S}$ are connected via

$$
\varphi(z)=\frac{1}{z} \frac{F(z)-F(0)}{F(z)+\overline{F(0)}}
$$

which induces a one-to-one correspondence between $\mathcal{C}$ and $\mathcal{S}$.

Finally we denote by $\mathcal{N}$ R. Nevanlinna's class of analytic functions $f(z): \mathbb{C}^{+} \rightarrow$ $\mathbb{C}^{+} \cup \mathbb{R}$. A function $f$ is in $\mathcal{N}$ if and only if it admits an integral representation

$$
f(z)=a+b z+\int_{\mathbb{R}} \frac{1+u z}{u-z} \tau(d u)=a+b z+\int_{\mathbb{R}}\left(\frac{1}{u-z}-\frac{u}{1+u^{2}}\right)\left(1+u^{2}\right) \tau(d u),
$$

where $b \geqslant 0, a \in \mathbb{R}$, and $\tau$ is finite nonnegative measure. Here $a, b$ and $\tau$ are uniquely determined by $f$. More precisely we have $a=\operatorname{Re} f(i)$ and $\tau(\mathbb{R})=$ $\operatorname{Im} f(i)-b$. From this formula it follows that

$$
f(z)=(b+o(1)) z
$$

for $z \in \mathbb{C}^{+}$such that $|\operatorname{Re} z| / \operatorname{Im} z$ stays bounded as $|z|$ tends to infinity. Hence if $b \neq 0$, then $f$ has a left inverse $f^{(-1)}$ defined on the region $\Gamma_{\alpha, \beta}$ defined (in Section 2) for any $\alpha>0$ and some positive $\beta=\beta(f, \alpha)$.

A function $f \in \mathcal{N}$ admits the representation

$$
f(z)=\int_{\mathbb{R}} \frac{\sigma(d u)}{u-z}, \quad z \in \mathbb{C}^{+},
$$

where $\sigma$ is a finite nonnegative measure, if and only if $\sup _{y \geqslant 1} y|f(i y)|<\infty$.

Note that the class $\mathcal{F}$ coincides with the subclass of Nevanlinna functions for which $f(z) / z \rightarrow 1$ as $z \rightarrow \infty$ nontangentially. Indeed, reciprocal Cauchy transforms of p-measures have obviously such property. Let $f \in \mathcal{N}$ and $f(z) / z \rightarrow$ 1 as $z \rightarrow \infty$ nontangentially. Then, by (3.4), $f$ admits the representation (3.3), where $b=1$. By (3.4) and (3.5), we see that $-1 / f(z)$ admits the representation 
(3.5), where $\sigma \in \mathcal{M}$. It follows from (3.3) with $b=1$ that a function $f \in \mathcal{F}$ admits the inequality

$$
\operatorname{Im} f(z) \geqslant \operatorname{Im} z, \quad z \in \mathbb{C}^{+} .
$$

The Stieltjes-Perron inversion formula for a function $f \in \mathcal{N}$ has the following form.

Let $\psi(u):=\int_{0}^{u}\left(1+t^{2}\right) \tau(d t)$. Then

$$
\psi\left(u_{2}\right)-\psi\left(u_{1}\right)=\lim _{\eta \rightarrow 0} \frac{1}{\pi} \int_{u_{1}}^{u_{2}} \operatorname{Im} f(\xi+i \eta) d \xi,
$$

where $u_{1}<u_{2}$ are continuity points of the function $\psi(u)$.

The following two results are due to Krein, M.

Theorem 3.1. The function $f(z)$ admits the representation

$$
f(z)=a+\int_{\mathbb{R}_{+}} \frac{\tau(d u)}{u-z}, \quad 0<\arg z<2 \pi,
$$

where $a \geqslant 0$ and $\tau$ is a nonnegative measure such that

$$
\int_{\mathbb{R}_{+}} \frac{\tau(d u)}{1+u}<\infty
$$

if and only if $f(z) \in \mathcal{N}$ and $f(z)$ is analytic and nonnegative on $(-\infty, 0)$.

Theorem 3.2. A function $f(z) \in \mathcal{N}$ be analytic and nonnegative on $(-\infty, 0)$ if and only if $z f(z)$ is in $\mathcal{N}$.

Corollary 3.3. (1) A function $f(z) \in \mathcal{N}$ is analytic and nonpositive on $(-\infty, 0)$ if and only if $f(z) / z$ is in $\mathcal{N}$.

(2) A function $f(z) / z$ admits a representation (3.8) with a nonnegative measure $\tau$ satisfying assumptions (3.9) and $\tau(\{0\})=0$ if and only if $f(z) \in \mathcal{K}$.

Proof. At first we shall prove the assertion (1). Let the function $f(z) \in \mathcal{N}$ be analytic and nonpositive on $(-\infty, 0)$. Then the function $-1 / f(z) \in \mathcal{N}$ and satisfies the assumptions of Theorem 3.2. By this theorem, $-z / f(z) \in \mathcal{N}$ and therefore $f(z) / z \in \mathcal{N}$. The converse assertion follows by repeating the previous arguments.

Let us now prove the assertion (2). It easy to see that $f(z) \in \mathcal{K}$ if the function $f(z) / z$ admits a representation (3.8) with a nonnegative measure $\tau$ which satisfies assumption (3.9) and $\tau(\{0\})=0$. The converse assertion follows from the assertion (1) of Corollary 3.3 and Theorem 3.1

Proposition 3.4. Let $f(z) \in \mathcal{N}$ be analytic and nonpositive on $(-\infty, 0)$. Then $f(z)$ is univalent in the left half-plane $i \mathbb{C}^{+}$. 
Proof. Since the function $f(z) / z$ admits representation (3.8), we see that

$$
f\left(z_{1}\right)-f\left(z_{2}\right)=\left(z_{1}-z_{2}\right)\left(a+\int_{(0, \infty)} \frac{u}{\left(u-z_{1}\right)\left(u-z_{2}\right)} \tau(d u)\right)
$$

for all $z_{1}, z_{2} \in \mathbb{C}^{+}$. Since for $z_{1} \neq z_{2}$ and $z_{1}, z_{2} \in \mathbb{C}^{+} \cap i \mathbb{C}^{+}$

$$
\begin{aligned}
\operatorname{Im} \int_{(0, \infty)} & \frac{u}{\left(u-z_{1}\right)\left(u-z_{2}\right)} \tau(d u) \\
& =\int_{(0, \infty)} \frac{-\left(\operatorname{Re} z_{1} \operatorname{Im} z_{2}+\operatorname{Re} z_{2} \operatorname{Im} z_{1}\right)+u\left(\operatorname{Im} z_{1}+\operatorname{Im} z_{2}\right)}{\left|u-z_{1}\right|^{2}\left|u-z_{2}\right|^{2}} u \tau(d u) \neq 0,
\end{aligned}
$$

we conclude from the preceding formula that $f\left(z_{1}\right) \neq f\left(z_{2}\right)$ for the considered $z_{1}$ and $z_{2}$. Hence $f(z)$ is univalent in $\mathbb{C}^{+} \cap i \mathbb{C}^{+}$. Since $f\left(\mathbb{C}^{+}\right) \subset \mathbb{C}^{+}$and $f$ is strictly increasing on $(-\infty, 0)$, the univalence of $f$ on $i \mathbb{C}^{+}$follows from the identity $f(\bar{z})=\overline{f(z)}$.

We need as well the following well-known result about Schur functions $Q_{\mu} \in \mathcal{S}_{*}$ (the definition of $Q_{\mu}$ see in (2.13) ).

Proposition 3.5. Let $\mu \in \mathcal{S}_{*}$, then

$$
Q_{\mu}(z)=z \frac{Q_{\mu}^{\prime}(0)+z \varphi_{1}(z)}{1+\overline{Q_{\mu}^{\prime}(0)} z \varphi_{1}(z)}
$$

where $\varphi_{1}(z) \in \mathcal{S}$.

We need the following result about the behavior of nondecreasing functions (see [16], Ch. 4, §16).

Proposition 3.6. Let $\nu$ be a nonnegative finite measure. If $\nu([a, b])>0$ and $\nu([a, x)), a<x<b$, is a continuous function, then there exists $[\alpha, \beta] \subset[a, b]$ such that $\nu([\alpha, \alpha+h])>$ ch and $\nu([\beta-h, \beta])>$ ch for all $0<h \leqslant h_{0}$, where $c>0$ and $h_{0}>0$ depend on the measure $\nu$.

We also need some results of the theory of real and complex variable (see 12 (1969), [19] (1965)).

Theorem 3.7. (Weierstrass' preparation theorem) Let $F(z, w)$ be a function of two complex variables which is analytic in neighborhood $\left|z-z_{0}\right|<r,\left|w-w_{0}\right|<\rho$ of the point $\left(z_{0}, w_{0}\right)$, and suppose that

$$
F\left(z_{0}, w_{0}\right)=0, \quad F\left(z_{0}, w\right) \not \equiv 0 .
$$

Then there is a neighborhood $\left|z-z_{0}\right|<r^{\prime}<r,\left|w-w_{0}\right|<\rho^{\prime}<\rho$ where $F(z, w)$ may be written as

$$
F(z, w)=\left(A_{0}(z)+A_{1}(z) w+\cdots+A_{k-1}(z) w^{k-1}+w^{k}\right) G(z, w),
$$


$k$ is determined by

$$
\frac{\partial F\left(z_{0}, w_{0}\right)}{\partial w}=\ldots \frac{\partial^{k-1} F\left(z_{0}, w_{0}\right)}{\partial w^{k-1}}=0, \quad \frac{\partial^{k} F\left(z_{0}, w_{0}\right)}{\partial w^{k}} \neq 0,
$$

the functions $A_{0}(z), A_{1}(z), \ldots, A_{k-1}(z)$ are analytic for $\left|z-z_{0}\right|<r^{\prime}$, and the function $G(z, w)$ is analytic and nonzero on the set $\left|z-z_{0}\right|<r^{\prime},\left|w-w_{0}\right|<\rho^{\prime}$.

Theorem 3.8. Let $\left\{u_{n}(z)\right\}_{n=1}^{\infty}$ denote a sequence of functions which are harmonic in a domain $B$. If $\left\{u_{n}(z)\right\}_{n=1}^{\infty}$ is uniformly bounded in the interior of $B$, it contains a subsequence that converges uniformly in the interior of $B$ to a harmonic function in $B$.

Theorem 3.9. Given a domain $B$ and a sequence $\left\{f_{n}(z)\right\}_{n=1}^{\infty}$ of regular functions in $B$, suppose the sequence $\left\{u_{n}(z)\right\}_{n=1}^{\infty}=\left\{\operatorname{Re} f_{n}(z)\right\}_{n=1}^{\infty}$ converges uniformly on every compact subset of $B$, and suppose $\left\{f_{n}(z)\right\}_{n=1}^{\infty}$ converges at some point $z_{0} \in B$. Then $\left\{f_{n}(z)\right\}_{n=1}^{\infty}$ converges uniformly on every compact subset of $B$ to a regular function.

Theorem 3.10. (Vitali) Let $\left\{f_{n}(z)\right\}_{n=1}^{\infty}$ denote a sequence function that are regular in B. Suppose that $\left\{f_{n}(z)\right\}_{n=1}^{\infty}$ is uniformly bounded in the interior of $B$ and converges on a set of points $z_{k} \in B, k=1,2, \ldots$, that has a cluster point in the interior of $B$. Then, the sequence $\left\{f_{n}(z)\right\}_{n=1}^{\infty}$ converges uniformly in the interior of $B$.

In the sequel we shall need the following two results of Bercovici and Voiculescu 4 .

Proposition 3.11. Let $\left\{\mu_{n}\right\}_{n=1}^{\infty}$ be a sequence of p-measures on $\mathbb{R}$. The following assertions are equivalent.

(1) The sequence $\left\{\mu_{n}\right\}_{n=1}^{\infty}$ converges weakly to a $p$-measure $\mu$.

(2) There exist $\alpha, \beta>0$ such that the sequence $\left\{\varphi_{\mu_{n}}\right\}_{n=1}^{\infty}$ converges uniformly on the compact subsets of $\Gamma_{\alpha, \beta}$ to a function $\varphi$, and $\varphi_{\mu_{n}}(i y)=o(y)$ uniformly in $n$ as $y \rightarrow+\infty$.

Moreover, if (1) and (2) are satisfied, we have $\varphi=\varphi_{\mu}$ in $\Gamma_{\alpha, \beta}$.

Proposition 3.12. Let $\left\{\mu_{n}\right\}_{n=1}^{\infty}$ be a tight sequence of p-measures on $\mathbb{R}_{+}$such that $\delta_{0}$ is not in the weak closure of $\left\{\mu_{n}\right\}_{n=1}^{\infty}$. The following assertions are equivalent.

(1) The sequence $\left\{\mu_{n}\right\}_{n=1}^{\infty}$ converges in the weak topology to a measure $\mu$.

(2) There exist numbers $\alpha \in(0, \pi)$ and $0<\beta<\Delta$ such that the sequence $\left\{\Sigma_{\mu_{n}}\right\}_{n=1}^{\infty}$ converges uniformly on $\Gamma_{\alpha, \beta, \Delta}^{+}$to a function $\Sigma$.

Moreover, if (1) and (2) are satisfied, we have $\Sigma=\Sigma_{\mu}$ in $\Gamma_{\alpha, \beta, \Delta}^{+}$.

We need the following result of Bercovici and Voiculescu [3] as well.

For positive number $\alpha$ denote $\mathbb{D}_{\alpha}:=\{z \in \mathbb{C}:|z|<\alpha\}$.

Proposition 3.13. Consider a measure $\mu \in \mathcal{M}_{*}$ and a sequence $\mu_{j} \in \mathcal{M}_{*}, j=$ $1, \ldots$ The following assertions are equivalent. 
(1) The sequence $\left\{\mu_{n}\right\}_{n=1}^{\infty}$ converges in the weak topology to a measure $\mu \in$ $\mathcal{M}_{*}$.

(2) There exists a positive number $\alpha$ such that the sequence $\left\{\Sigma_{\mu_{j}}\right\}_{j=1}^{\infty}$ converges uniformly on $\mathbb{D}_{\alpha}$ to a function $\Sigma_{\mu}$.

Moreover, if (1) and (2) are satisfied, we have $\Sigma=\Sigma_{\mu}$ in $\mathbb{D}_{\alpha}$.

Finally we need some results on Delfic semigroups which will be used in Section 6.

In the paper we consider metric semigroups only. For such semigroups we use Davidson's version [9] of the definition of Delphic semigroups.

Definition 3.14. A semigroup $G$ (under a product semigroup operation ·) is called Delphic if $G$ is commutative, has an identity $e$, and satisfies the following conditions:

(1) There exist a set $M \subset \prod_{j=1}^{\infty} G$ and map $l$ of $M$ into $G$. ( $M$ is the set of sequences of elements of $G$ that possess limits, and $l$ is the operation of taking the limit.)

(2) If the sequence $\left\{u_{n}\right\}$ is in $M$ and $\left\{n^{\prime}\right\}$ is a subsequence of $\{n\}$, then $\left\{u_{n^{\prime}}\right\}$ is also in $M$ and $l\left\{u_{n^{\prime}}\right\}=l\left\{u_{n}\right\}$.

(3) Let $u$ be arbitrary in $G$. If $\left\{u_{n}\right\}$ is such that for every subsequence $\left\{n^{\prime}\right\}$ of $\{n\}$ there is a sub-subsequence $\left\{n^{\prime \prime}\right\}$ of $\left\{n^{\prime}\right\}$ with $\left\{u_{n^{\prime \prime}}\right\}$ in $M$ and $l\left\{u_{n^{\prime \prime}}\right\}=u$, then $\left\{u_{n}\right\}$ is itself in $M$ and $l\left\{u_{n}\right\}=u$.

(4) If $\left\{u_{n}\right\}$ and $\left\{v_{n}\right\}$ are in $M$, then so is $\left\{u_{n} \cdot v_{n}\right\}$ and $l\left\{u_{n} \cdot v_{n}\right\}=l\left\{u_{n}\right\} l\left\{v_{n}\right\}$.

(5) There is a homomorphism $D$ of $G$ into $\mathbb{R}_{+}$under addition, such that if $\left\{u_{n}\right\}$ in $M$ then the limit $\lim _{n \rightarrow \infty} D\left(u_{n}\right)$ exists and equals $D\left(l\left\{u_{n}\right\}\right)$.

(6) $D(u)=0$ if and only if $u=e$.

(7) If for every $n u_{n}$ is a factor of $u$, then there is a subsequence $\left\{n^{\prime}\right\}$ of $\{n\}$ such that $\left\{u_{n^{\prime}}\right\}$ is in $M$ and $l\left\{u_{n^{\prime}}\right\}$ is a factor of $u$.

(8) Suppose that $\left\{u_{n j}\right\}, 1 \leqslant j \leqslant n<\infty$, denotes a triangular array such that $\max _{1 \leqslant j \leqslant n} D\left(u_{n j}\right) \rightarrow 0$ as $n \rightarrow \infty$. Let $u_{n}=\prod_{j=1}^{n} u_{n j}$ for each $n$. If the sequence $\left\{u_{n}\right\}$ of row-products is in $M$, then $l\left\{u_{n}\right\}$ is infinitely divisible.

In the sequel we denote by $I(G)$ the set of i.d. elements of the group $G$. There are three main theorems for such Delphic semigroups:

Theorem 3.15. (Davidson (1968))

(1) Every infinitely divisible element can be represented as the limit of a triangular array (for definition see (8) above).

(2) In every Delphic semigroup there are three exclusive and exhaustive classes:

(a) the indecomposable elements

(b) the decomposable elements which admit an indecomposable factor

(c) the infinitely divisible elements which have no indecomposable factors (a set we shall denote by $I_{0}$ ). 
(3) For each $u$ in the semigroup there is at least one representation $u=$ $w \cdot \prod v_{j}$, where $w$ is in $I_{0}$ and each $v_{j}$ is indecomposable. The product is at most countable and may be finite or void.

Definition 3.16. Let $G$ be a semigroup and let $H$ be a non-empty subsemigroup of $G$. $H$ is said to be hereditary in $G$ if for every $u$ in $H$, the set of factors of $u$ in $G$, say $F(u)$, satisfies $F(u) \subset H$.

In the sequel we need the following assertions about Delphic semigroups (see [9]).

Proposition 3.17. Let $G$ be a commutative metric semigroup, and let $\left(G_{a}\right)$ (a in some index set $A$ ) be a collection of hereditary subsemigroups of $G$ that together cover $G$. If every $G_{a}$ is Delphic then Theorem 3.15 of this section holds for $G$.

Proposition 3.18. Under the conditions of Proposition 3.17 suppose that for all a in $A, G_{a}$ is open in $G$. Let $(u(j, l)), 1 \leqslant l \leqslant j<\infty$, be a triangular array of elements of $G$ whose row products $u(j)=\prod_{l=1}^{j} u(j, l)$ converge to $u$ in $G$ as $j \rightarrow \infty$. Assume that $u \in G_{b}$ for some $b \in A$. If $\lim _{j \rightarrow \infty} \max _{1 \leqslant l \leqslant j} D_{b}(u(j, l))=0$, then $u$ is infinitely divisible in $G$.

\section{AdDitive FREE CONVOLUTION}

In this section we prove Theorem 2.1 and its consequences. We need the following auxiliary results.

Lemma 4.1. Let $g: \mathbb{C}^{+} \rightarrow \mathbb{C}^{-}$be analytic with

$$
\liminf _{y \rightarrow+\infty} \frac{|g(i y)|}{y}=0 .
$$

Then the function $f: \mathbb{C}^{+} \rightarrow \mathbb{C}$ defined via $z \mapsto z+g(z)$ takes every value in $\mathbb{C}^{+}$ precisely once. The inverse $f^{(-1)}: \mathbb{C}^{+} \rightarrow \mathbb{C}^{+}$thus defined is in the class $\mathcal{F}$.

This lemma generalizes a result of Maassen 18 (see Lemma 2.3). Maassen proved Lemma 4.1 under the additional restriction $|g(z)| \leqslant c / \operatorname{Im} z$ for $z \in \mathbb{C}^{+}$, where $c$ is a constant.

Proof. Since $g: \mathbb{C}^{+} \rightarrow \mathbb{C}^{-}$is analytic, it can be written in Nevanlinna's integral form (see (3.3) in Section 3)

$$
g(z)=a-b z-\int_{\mathbb{R}} \frac{1+u z}{u-z} \sigma(d u),
$$

where $a, b \in \mathbb{R}, b \geqslant 0$ and $\sigma$ is a finite nonnegative measure. By (4.1), $b=0$. Denote $\alpha_{c}:=\sigma(\{|u|>c\})$, where $c>0$ is chosen such that $\alpha_{c}<1$. We may decompose $1+u z$ in the integral (4.2) as $1+u^{2}+u(z-u)$ for $|u| \leqslant c$ and as 
$1+z^{2}+z(u-z)$ for $|u|>c$. Hence we get $f(z)=a_{c}+f_{1}(z)+f_{2}(z)$ for $z \in \mathbb{C}^{+}$, where $a_{c}:=a+\int_{[-c, c]} u \sigma(d u)$ and

$$
f_{1}(z):=\left(1-\alpha_{c}\right) z-\int_{[-c, c]} \frac{1+u^{2}}{u-z} \sigma(d u), \quad f_{2}(z):=-\left(1+z^{2}\right) \int_{|u|>c} \frac{\sigma(d u)}{u-z}
$$

Let $w \in \mathbb{C}^{+}$, denote $w_{1}:=w-a_{c}$. For every fixed $w \in \mathbb{C}^{+}$we consider a closed rectifiable curve $\gamma_{1}=\gamma_{1}(w)$ (see Figure 1) consisting of some smooth curve $\gamma_{1,1}$ connecting $w_{1}-R$ to $w_{1}+R$ inside the strip $0<\operatorname{Im} z<\operatorname{Im} w$, the arc $\gamma_{1,2}: 0<\arg \left(z-w_{1}\right) \leqslant \eta$ on the circle $\left|z-w_{1}\right|=R$ connecting $w_{1}+R$ to $w_{1}+R e^{i \eta}$, the $\operatorname{arc} \gamma_{1,3}: \eta<\arg \left(z-w_{1}\right) \leqslant \pi-\eta$ on the circle $\left|z-w_{1}\right|=R$ connecting $w_{1}+R e^{i \eta}$ to $w_{1}-R e^{-i \eta}$, and the $\operatorname{arc} \gamma_{1,4}: \pi-\eta<\arg \left(z-w_{1}\right) \leqslant \pi$ on the circle $\left|z-w_{1}\right|=R$ connecting $w_{1}-R e^{-i \eta}$ to $w_{1}-R$. Here $\eta$ is given by $\eta:=10^{-2} \min \left\{\arg w_{1},-\arg \bar{w}_{1}\right\}$. We also assume that $R>0$ is sufficiently large.

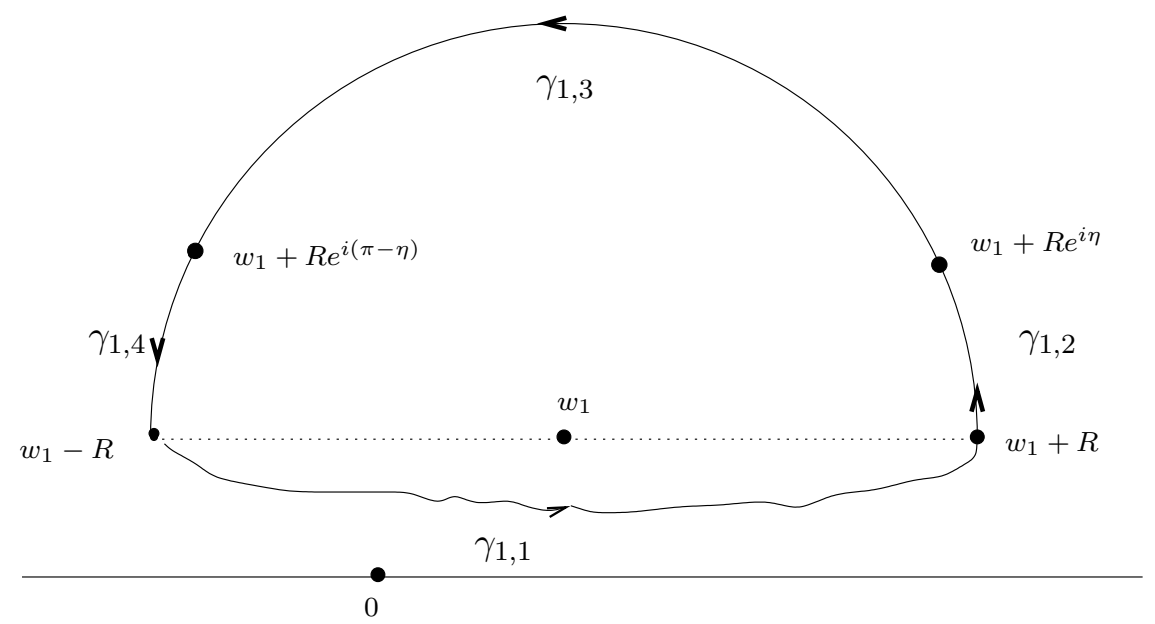

FigURE 1.

Note by (4.2) with $b=0$ that $\max _{z \in \gamma_{1,3}}|g(z)| /|z| \rightarrow 0$ as $R \rightarrow \infty$. We also see that on $\gamma_{1,3} \operatorname{Im} z \geqslant \operatorname{Im} w+R \sin \eta$. Since $-\operatorname{Im} g(z)=o(R), z \in \gamma_{1,3}$, we have $\operatorname{Im} f(z) \geqslant \operatorname{Im} w+R \sin \eta-o(R)>\operatorname{Im} w, z \in \gamma_{1,3}$. Therefore, if $z$ runs through $\gamma_{1,3}$ the image $f(z)$ lies in the half-plane $\operatorname{Im} z>\operatorname{Im} w$.

For $z \in \gamma_{1,1}, \operatorname{Im}(z+g(z))<\operatorname{Im} z \leqslant \operatorname{Im} w$. Therefore, if $z$ runs through $\gamma_{1,1}$ the image $f(z)$ lies in the half-plane $\operatorname{Im} z<\operatorname{Im} w$.

Let $z \in \gamma_{1,2}$. It is easy to see that, for $|z|>c$,

$$
f_{1}(z)=\left(1-\alpha_{c}\right) z\left(1+\frac{1}{1-\alpha_{c}} \sum_{k=1}^{\infty} \frac{1}{z^{k+1}} \int_{[-c, c]} u^{k-1}\left(1+u^{2}\right) \sigma(d u)\right)
$$


Therefore we obtain the following formula, for $|z|>2\left(c^{2}+1\right)(\sigma(\mathbb{R})+1) /\left(1-\alpha_{c}\right)$,

$$
\log f_{1}(z)=\log \left(1-\alpha_{c}\right)+\log z+\sum_{m=1}^{\infty} \frac{a_{m}}{z^{m+1}}
$$

where $a_{m}$ are real coefficients such that $\left|a_{m}\right| \leqslant K^{m}, m=1, \ldots$, with some positive constant $K$. Here and in the sequel we choose the principle branch of the logarithm. Hence, for $|z|>2 K$,

$$
\begin{aligned}
\arg f_{1}(z) & =\arg z-\sum_{m=1}^{\infty} \frac{a_{m} \sin ((m+1) \arg z)}{|z|^{m+1}} \\
& =\left(1+\Theta \sum_{m=1}^{\infty} \frac{K^{m}(m+1)}{|z|^{m+1}}\right) \arg z=\left(1+6 \Theta \frac{K}{|z|^{2}}\right) \arg z,
\end{aligned}
$$

where $\Theta$ denotes a real-valued quantity such that $|\Theta| \leqslant 1$. On the other hand we easily obtain, for $|z|>2$,

$$
\arg \left(1+z^{2}\right)=2\left(1+\frac{2 \Theta}{|z|^{2}}\right) \arg z
$$

Therefore we conclude from the definition of $f_{2}(z)$, taking into account that $-\int_{|u|>c} \frac{\sigma(d u)}{u-z} \in \mathbb{C}^{-}$,

$$
-\pi+2\left(1+\frac{2 \Theta}{|z|^{2}}\right) \arg z \leqslant \arg f_{2}(z) \leqslant 2\left(1+\frac{2 \Theta}{|z|^{2}}\right) \arg z, \quad z \in \gamma_{1,2} .
$$

From (4.3) and (4.4) it follows that, for $z \in \gamma_{1,2},\left|\arg f_{1}(z)-\arg f_{2}(z)\right|<\pi$ and therefore

$$
-\pi+2\left(1+\frac{2 \Theta}{|z|^{2}}\right) \arg z \leqslant \arg \left(f_{1}(z)+f_{2}(z)\right) \leqslant 2\left(1+\frac{2 \Theta}{|z|^{2}}\right) \arg z, \quad z \in \gamma_{1,2} .
$$

We conclude from (4.5) that the image $\zeta=f(z)$ lies in the domain $D_{1}:=\{\zeta \in$ $\mathbb{C}:-\pi<\arg \zeta<3 \eta\}$ when $z$ runs through $\gamma_{1,2}$. In addition the point $w$ does not lie in $D_{1}$ by the choice of the parameter $\eta$. In the same way we deduce that the image $\zeta=f(z)$ lies in $D_{2}:=\{\zeta \in \mathbb{C}: \pi-3 \eta<\arg \zeta<2 \pi\}$ and $w \notin D_{2}$ when $z$ runs through $\gamma_{1,4}$.

Hence $f(z)$ winds around $w$ once, and it follows from the argument principle that inside the curve $\gamma_{1}$ there is a unique point $z_{0}$ such that $f\left(z_{0}\right)=w$. Since this relation holds for all sufficiently large $R>0$ and all curves $\gamma_{1,1}$, we deduce that the point $z_{0}$ is unique in $\mathbb{C}^{+}$.

Hence the inverse function $f^{(-1)}: \mathbb{C}^{+} \rightarrow \mathbb{C}^{+}$exists and is analytic in $\mathbb{C}^{+}$. By condition (4.1) $\lim _{y \rightarrow+\infty}\left(i y / f^{(-1)}(i y)\right)=1$ and therefore $f^{(-1)} \in \mathcal{F}$. This proves the lemma.

Let $z_{1} \in \mathbb{C}^{+}$and $z_{2} \in \mathbb{C}^{+}$, and introduce the functions

$$
w_{1}\left(z_{1}, z_{2}\right):=z_{1}+z_{2}-F_{\mu_{2}}\left(z_{2}\right), \quad w_{2}\left(z_{1}, z_{2}\right):=z_{1}+z_{2}-F_{\mu_{1}}\left(z_{1}\right) .
$$


Lemma 4.2. For every $z \in \mathbb{C}^{+}$there exist unique points $z_{1} \in \mathbb{C}^{+}$and $z_{2} \in \mathbb{C}^{+}$ such that

$$
z=w_{1}\left(z_{1}, z_{2}\right) \quad \text { and } \quad z=w_{2}\left(z_{1}, z_{2}\right) .
$$

Proof. Let us fix $z \in \mathbb{C}^{+}$. For every $z_{2} \in \mathbb{C}^{+}$we define $z_{1}:=z-\left(z_{2}-F_{\mu_{2}}\left(z_{2}\right)\right)$. Recall that $F_{\mu_{j}} \in \mathcal{F}, j=1$, 2. Since, by (3.6),$\left(z_{2}-F_{\mu_{2}}\left(z_{2}\right)\right) \in \mathbb{C}^{-} \cup \mathbb{R}$, it follows that $z_{1} \in \mathbb{C}^{+}$. Hence it suffices to prove that the equation

$$
F_{\mu_{2}}\left(z_{2}\right)=F_{\mu_{1}}\left(z-\left(z_{2}-F_{\mu_{2}}\left(z_{2}\right)\right)\right)
$$

has a unique solution $z_{2} \in \mathbb{C}^{+}$. Rewrite (4.7) in the form

$$
z=z_{2}-g_{\mu_{1}}\left(z-\left(z_{2}-F_{\mu_{2}}\left(z_{2}\right)\right)\right),
$$

where $g_{\mu_{1}}(w):=F_{\mu_{1}}(w)-w$ for $w \in \mathbb{C}^{+}$. We shall prove that

$$
\left|g_{\mu_{1}}\left(z-\left(i \operatorname{Im} z_{2}-F_{\mu_{2}}\left(i \operatorname{Im} z_{2}\right)\right)\right)\right| / \operatorname{Im} z_{2} \rightarrow 0 \quad \text { as } \quad \operatorname{Im} z_{2} \rightarrow+\infty .
$$

Let to the contrary

$$
\mid g_{\mu_{1}}\left(z-\left(i y_{k}-F_{\mu_{2}}\left(i y_{k}\right)\right) \mid / y_{k} \geqslant c>0\right.
$$

for some sequence $\left\{y_{k}\right\}_{k=1}^{\infty}$ such that $y_{k} \geqslant 1$ and $y_{k} \rightarrow+\infty$, and for a constant $c$.

If liminf $\operatorname{in}_{y_{k} \rightarrow+\infty}\left|i y_{k}-F_{\mu_{2}}\left(i y_{k}\right)\right|<\infty$, then there exists a subsequence $\left\{y_{k}^{\prime}\right\}_{k=1}^{\infty} \subset$ $\left\{y_{k}\right\}_{k=1}^{\infty}$ such that $\lim _{y_{k}^{\prime} \rightarrow+\infty}\left|i y_{k}^{\prime}-F_{\mu_{2}}\left(i y_{k}^{\prime}\right)\right|<\infty$. It is easy to see, $\operatorname{Im}\left(z-\left(i y_{k}^{\prime}-\right.\right.$ $\left.F_{\mu_{2}}\left(i y_{k}^{\prime}\right)\right) \geqslant \operatorname{Im} z$ and $\mid z-\left(i y_{k}^{\prime}-F_{\mu_{2}}\left(i y_{k}^{\prime}\right)|\leqslant| z \mid+c_{1}\right.$ for all $y_{k}$ and for some constant $c_{1}>0$. Hence in this case we have $\mid g_{\mu_{1}}\left(z-\left(i y_{k}^{\prime}-F_{\mu_{2}}\left(i y_{k}^{\prime}\right)\right) \mid \leqslant c_{2}\right.$ for all $y_{k}^{\prime}$ and for some constant $c_{2}>0$. This estimate contradicts to (4.9).

Let $\liminf \operatorname{ink}_{y_{k} \rightarrow+\infty}\left|i y_{k}-F_{\mu_{2}}\left(i y_{k}\right)\right|=\infty$. Since $\left|g_{\mu_{1}}(z+i \operatorname{Im} w)\right| / \operatorname{Im} w \rightarrow 0$ as $\operatorname{Im} w \rightarrow+\infty$ and $\left|i \operatorname{Im} z_{2}-F_{\mu_{2}}\left(i \operatorname{Im} z_{2}\right)\right| / \operatorname{Im} z_{2} \rightarrow 0$ as $\operatorname{Im} z_{2} \rightarrow+\infty$, we see that

$$
\frac{\left|g_{\mu_{1}}\left(z-\left(i y_{k}-F_{\mu_{2}}\left(i y_{k}\right)\right)\right)\right|}{\left|z-\left(i y_{k}-F_{\mu_{2}}\left(i y_{k}\right)\right)\right|} \frac{\left|z-\left(i y_{k}-F_{\mu_{2}}\left(i y_{k}\right)\right)\right|}{y_{k}} \rightarrow 0 \text { as } \quad y_{k} \rightarrow+\infty,
$$

a contradiction with (4.9). Hence (4.8) is proved.

Consider the function $\tilde{f}\left(z_{2}\right):=z_{2}-\tilde{g}\left(z_{2}\right)$, where $\tilde{g}\left(z_{2}\right):=g_{\mu_{1}}\left(z-\left(z_{2}-F_{\mu_{2}}\left(z_{2}\right)\right)\right)$, $z_{2} \in \mathbb{C}^{+}$. By the definition of the function $g_{\mu_{1}}$, we see that $-\tilde{g}: \mathbb{C}^{+} \rightarrow \mathbb{C}^{-}$. By (4.8), the $-\tilde{g}$ satisfies the condition (4.1). Applying Lemma 4.1 to the function $\tilde{f}\left(z_{2}\right)$, we obtain that (4.7) has a unique solution $z_{2} \in \mathbb{C}^{+}$for every fixed $z \in \mathbb{C}^{+}$, thus proving the lemma.

Proof of Theorem 2.1. Consider the function $F\left(z, z_{2}\right):=F_{\mu_{2}}\left(z_{2}\right)-F_{\mu_{1}}\left(z-\left(z_{2}-\right.\right.$ $\left.\left.F_{\mu_{2}}\left(z_{2}\right)\right)\right)$ as a function of the two complex variables $z$ and $z_{2}$. It is analytic on $\mathbb{C}^{+} \times \mathbb{C}^{+}$. By Lemma 4.2, for every fixed $z=z^{0} \in \mathbb{C}^{+}$equation (4.7) has an unique solution $z_{2}^{0} \in \mathbb{C}^{+}$. Hence $F\left(z^{0}, z_{2}^{0}\right)=0$. We shall verify that $F\left(z^{0}, z_{2}\right) \not \equiv 0$ for $z_{2} \in$ $\mathbb{C}^{+}$. Assume that $F\left(z^{0}, z_{2}\right) \equiv 0$ holds in $z_{2} \in \mathbb{C}^{+}$. Since $\operatorname{Im} F_{\mu_{2}}(i y) / y \rightarrow 1$ and $\operatorname{Im} F_{\mu_{1}}\left(z-\left(i y-F_{\mu_{2}}(i y)\right)\right) / y \rightarrow 0$ as $y \rightarrow+\infty$, by arguments as in Lemma 4.2 , we arrive at a contradiction. Therefore the function $F\left(z, z_{2}\right)$ satisfies the assumption (3.10) of Theorem 3.7 (Weierstrass' preparation theorem) at the point $\left(z^{0}, z_{2}^{0}\right)$. 
Moreover, by this theorem, the function $F\left(z, z_{2}\right)$ admits the representation (3.11) in a neighborhood $\left|z-z^{0}\right|<r^{\prime},\left|z_{2}-z_{2}^{0}\right|<\rho^{\prime}$ with some positive integer $k$. Let us show that there exists $0<r^{\prime \prime} \leqslant r^{\prime}$ such that the equation $F\left(z, z_{2}\right)=0$ has a unique root $z_{2}$ in $\left|z_{2}-z_{2}^{0}\right|<\rho^{\prime}$ for any given $z$ with $\left|z-z^{0}\right|<r^{\prime \prime}$. Since $F\left(z, z_{2}\right)=0$, 3.11) implies that

$$
P\left(z, z_{2}\right):=A_{0}(z)+A_{1}(z) z_{2}+\cdots+A_{k-1}(z) z_{2}^{k-1}+z_{2}^{k}=0
$$

for $z, z_{2}$ from the above neighborhood. Here the functions $A_{0}(z), \ldots, A_{k-1}(z)$ are analytic in the domain $\left|z-z^{0}\right|<r^{\prime \prime}$. For every $z$ with $\left|z-z^{0}\right|<r^{\prime \prime}$ and a sufficiently small $r^{\prime \prime}=r^{\prime \prime}\left(z^{0}\right) \in\left(0, r^{\prime}\right)$, this equation has $k$ roots $z_{2,1}(z), \ldots z_{2, k}(z)$ in $\left|z_{2}-z_{2}^{0}\right|<\rho^{\prime}$, some of which may be multiple roots. By Lemma 4.2, $z_{2,1}(z)=$ $\cdots=z_{2, k}(z)$ for all $\left|z-z^{0}\right|<r^{\prime \prime}$ and hence $P\left(z, z_{2}\right)=\left(z_{2}-z_{2,1}(z)\right)^{k}=0$, $\left|z-z^{0}\right|<r^{\prime \prime}$. We conclude that, for $\left|z-z^{0}\right|<r^{\prime \prime}$, the solution of equation (4.7) has the form $z_{2}=z_{2,1}(z)$, where $z_{2,1}(z)=-A_{k-1}(z) / k$ is an analytic function.

Thus, we have proved that for every given point $z^{0} \in \mathbb{C}^{+}$there exists a neighborhood $\left|z-z^{0}\right|<r^{\prime \prime}\left(z^{0}\right)$ such that (4.7) has an unique regular solution $z_{2}=z_{2}\left(z ; z^{0}\right)$ with values in $\mathbb{C}^{+}$. Note that for points $z^{\prime} \in \mathbb{C}^{+}$and $z^{\prime \prime} \in \mathbb{C}^{+}, z^{\prime} \neq z^{\prime \prime}$, we have $z_{2}\left(z ; z^{\prime}\right)=z_{2}\left(z ; z^{\prime \prime}\right)$ for all $z$ in $\left\{\left|z-z^{\prime}\right|<r^{\prime \prime}\left(z^{\prime}\right)\right\} \cap\left\{\left|z-z^{\prime \prime}\right|<r^{\prime \prime}\left(z^{\prime \prime}\right)\right\}$. By the monodromy theorem (see [19], v. 3, p. 269, 20], p. 217), there exists a regular function $Z_{2}(z), z \in \mathbb{C}^{+}$, such that, for every point $z^{0} \in \mathbb{C}^{+}, Z_{2}(z)=z_{2}\left(z ; z^{0}\right)$ for $\left|z-z^{0}\right|<r^{\prime \prime}\left(z^{0}\right)$. Therefore $Z_{2}(z) \in \mathcal{N}$ and it is an unique solution of (4.7).

It is easy to see from (4.7) that $Z_{2}(z)$ is in the class $\mathcal{F}$. Indeed, we note that the function $F_{\mu_{2}}\left(Z_{2}(z)\right)-Z_{2}(z) \in \mathcal{N}$ and, by (3.4), $F_{\mu_{2}}\left(Z_{2}(z)\right)-Z_{2}(z)=$ $(b+o(1)) z$, where $b \geqslant 0$ is some constant, for $z \in \mathbb{C}^{+}$such that $|\operatorname{Re} z| / \operatorname{Im} z$ stays bounded as $|z|$ tends to infinity. Hence the function $z+F_{\mu_{2}}\left(Z_{2}(z)\right)-$ $Z_{2}(z) \in \mathcal{N}$ and $\left|z+F_{\mu_{2}}\left(Z_{2}(z)\right)-Z_{2}(z)\right| \rightarrow \infty$ for the same $z$. In addition $\left|\operatorname{Re}\left(z+F_{\mu_{2}}\left(Z_{2}(z)\right)-Z_{2}(z)\right)\right| / \operatorname{Im}\left(z+F_{\mu_{2}}\left(Z_{2}(z)\right)-Z_{2}(z)\right)$ remains bounded as $|z|$ tends to infinity. Therefore, by (3.4),

$$
F_{\mu_{1}}\left(z+F_{\mu_{2}}\left(Z_{2}(z)\right)-Z_{2}(z)\right)=\left(z+F_{\mu_{2}}\left(Z_{2}(z)\right)-Z_{2}(z)(1+o(1))\right.
$$

for the considered $z$. Using this relation, we conclude from (4.7) and (3.4) that

$$
Z_{2}(z)=(1+o(1)) z+o(1)\left(F_{\mu_{1}}\left(Z_{2}(z)\right)-Z_{2}(z)\right)=(1+o(1)) z
$$

for the same $z$. Thus $Z_{2} \in \mathcal{F}$. The desired result is proved.

Choosing $Z_{1}(z):=z-Z_{2}(z)+F_{\mu_{2}}\left(Z_{2}(z)\right), z \in \mathbb{C}^{+}$, we see that $Z_{1}$ and $Z_{2}$ are unique solutions of (4.6) in the class $\mathcal{F}$. Hence Theorem 2.1 is proved.

Proof of Corollary 2.2. For simplicity we shall prove this corollary in the case $n=3$. The general case follows by induction.

Denote $\mu_{2,3}:=\mu_{2} \boxplus \mu_{3}$. We have, by associativity, $\mu_{1} \boxplus \mu_{2} \boxplus \mu_{3}=\mu_{1} \boxplus \mu_{2,3}$. By Theorem 2.1, there exist unique functions $W_{1}$ and $W_{2,3}$ in the class $\mathcal{F}$ such that, for $z \in \mathbb{C}^{+}$,

$$
z=W_{1}(z)+W_{2,3}(z)-F_{\mu_{1}}\left(W_{1}(z)\right) \quad \text { and } \quad F_{\mu_{1}}\left(W_{1}(z)\right)=F_{\mu_{2,3}}\left(W_{2,3}(z)\right)
$$


On the other hand, again by Theorem 2.1, there exist unique functions $W_{2} \in \mathcal{F}$ and $W_{3} \in \mathcal{F}$ such that, for $z \in \mathbb{C}^{+}$,

$$
z=W_{2}(z)+W_{3}(z)-F_{\mu_{2}}\left(W_{2}(z)\right) \quad \text { and } \quad F_{\mu_{2}}\left(W_{2}(z)\right)=F_{\mu_{3}}\left(W_{3}(z)\right) .
$$

Hence, replacing $z$ by $W_{2,3}(z)$ in the last equation we get, for all $z \in \mathbb{C}^{+}$,

$$
W_{2,3}(z)=W_{2}\left(W_{2,3}(z)\right)+W_{3}\left(W_{2,3}(z)\right)-F_{\mu_{2}}\left(W_{2}\left(W_{2,3}(z)\right)\right)
$$

and

$$
F_{\mu_{2}}\left(W_{2}\left(W_{2,3}(z)\right)\right)=F_{\mu_{3}}\left(W_{3}\left(W_{2,3}(z)\right)\right) .
$$

Comparing (4.10) and (4.11), (4.12), we obtain the assertion of Corollary 2.2 with $Z_{1}(z)=W_{1}(z), Z_{2}(z)=W_{2}\left(W_{2,3}(z)\right)$ and $Z_{3}(z)=W_{3}\left(W_{2,3}(z)\right)$.

Corollary 2.3 is an obvious consequence of Corollary 2.2. Note that the continuous semigroup version of Corollary 2.3 with $t \geqslant 1$ replacing $n$ is proved using Lemma 4.1 and Lemma 4.2, and repeating the arguments of Theorem 2.1.

\section{Multiplicative free Convolution}

1. Consider the case of multiplicative convolution for p-measures of class $\mathcal{M}_{+}$. In order to prove Theorem 2.4 we need the following two auxiliary results.

Let $\mu_{1}, \mu_{2} \in \mathcal{M}_{+}$and $w \in \mathbb{C}^{+}$. Introduce the functions

$$
f_{1}(z)=w \frac{R_{\mu_{1}}(z)}{z}, \quad f_{2}(z)=\frac{R_{\mu_{2}}\left(f_{1}(z)\right)}{f_{1}(z)}, \quad \text { and } \quad f_{3}(z)=\frac{z}{f_{2}(z)}, \quad z \in \mathbb{C}^{+} .
$$

Lemma 5.1. The function $f_{3}: \mathbb{C}^{+} \rightarrow \mathbb{C}$ takes the value $w \in \mathbb{C}^{+}$precisely once. Moreover, $f_{3}$ takes this value in $D_{w}:=\{z \in \mathbb{C}: \arg w \leqslant \arg z<\pi\}$.

Proof. We shall fix $w \in \mathbb{C}^{+}$. Let $\alpha \in(0, \arg w)$. Denote by $\gamma_{2}=\gamma_{2}(\alpha)$ (see Figure 2) the closed rectifiable curve consisting of the line segment $\gamma_{2,1}$ : $t e^{i \alpha}, 1 / R \leqslant t \leqslant R$, connecting $e^{i \alpha} / R$ to $R e^{i \alpha}$, the $\operatorname{arc} \gamma_{2,2}: \alpha<\arg z<\pi$ on the circle $|z|=R$ connecting $R e^{i \alpha}$ to $-R$, the line segment $\gamma_{2,3}:-R \leqslant t \leqslant-1 / R$ connecting $-R$ to $-1 / R$, and the arc $\gamma_{2,4}: \alpha<\arg z<\pi$ on the circle $|z|=1 / R$ connecting $-1 / R$ to $e^{i \alpha} / R$. Here the parameter $R>0$ and will be chosen later sufficiently large. Let $z$ run through $\gamma_{2}$ in the counter clockwise direction.

Since the function $R_{\mu_{1}}$ is in the Krein class $\mathcal{K}$, we note that $\arg w \leqslant \arg f_{1}(z)<$ $\pi$ for all $z \in \mathbb{C}$ with $\arg w \leqslant \arg z \leqslant \pi$. Let $z \in \mathbb{C}$ such that $\arg w<\arg z<$ $\pi+\arg w$. Since $R_{\mu_{1}}(\bar{z})=\overline{R_{\mu_{1}}(z)}$ and $0 \leqslant \arg \left(R_{\mu_{1}}(z) / z\right)<\pi-\arg z$ for $z \in \mathbb{C}^{+}$, we conclude that $\operatorname{Im} f_{1}(z)>0$ on the angular domain $\{z \in \mathbb{C}: \arg w<\arg z<$ $\pi+\arg w\}$. Therefore the function $f_{2}\left(z e^{i \arg w}\right)$ is in the class $\mathcal{N}$.

On the other hand we see that $\arg w \leqslant \arg f_{1}(z)<\pi+\arg w$ for all $z \in \mathbb{C}^{+}$. Since $R_{\mu_{2}}(\bar{z})=\overline{R_{\mu_{2}}(z)}$ and $0 \leqslant \arg \left(R_{\mu_{2}}(z) / z\right)<\pi-\arg z$ for $z \in \mathbb{C}^{+}$, we deduce that $e^{i \arg w} f_{2}(z)$ is in the class $\mathcal{N}$.

Using representation (3.3) for functions $f \in \mathcal{N}$, we note that if $\left|f\left(r e^{i \alpha}\right)\right| / r \rightarrow 0$ as $r \rightarrow \infty$ for any fixed $\alpha \in(0, \pi)$, then $|f(z)| / r \rightarrow 0$ as $r \rightarrow \infty$ uniformly in 


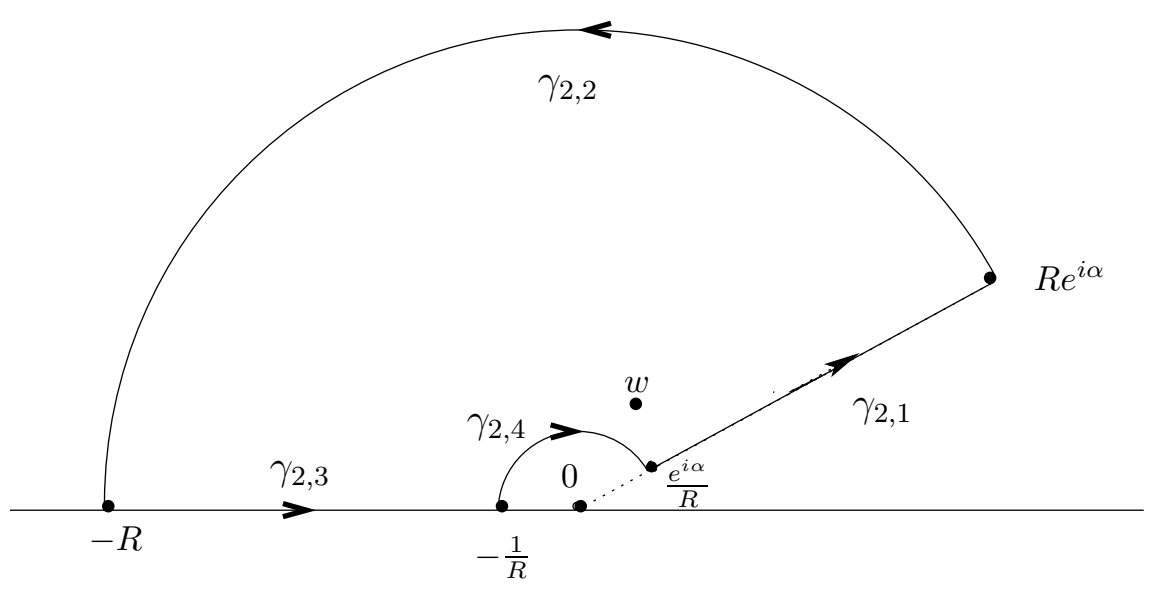

FiguRE 2.

the angle $\delta \leqslant \arg z \leqslant \pi-\delta$ with any fixed $\delta \in(0, \pi)$. From this and from the relations $f_{2}\left(z e^{i \arg w}\right) \in \mathcal{N}$ and $e^{i \arg w} f_{2}(z) \in \mathcal{N}$ we easily obtain that the estimate

$$
\max _{z \in \gamma_{2,2}} \frac{\left|f_{2}(z)\right|}{R} \rightarrow 0, \quad R \rightarrow \infty
$$

follows from the estimate

$$
\frac{\left|f_{2}(-R)\right|}{R} \rightarrow 0, \quad R \rightarrow \infty
$$

Let us prove (5.2). At first step we shall consider the behavior of the function $f_{1}(-R)$ for $R \geqslant 1$. The functions $R_{\mu_{j}} \in \mathcal{K}, j=1,2$, admit (see (2.8)) the representation

$$
R_{\mu_{j}}(z)=a_{j} z+z \int_{(0, \infty)} \frac{\tau_{j}(d u)}{u-z}:=a_{j} z+z g_{j}(-z), \quad z \in \mathbb{C}^{+},
$$

where $a_{j} \geqslant 0$ and the nonnegative measures $\tau_{j}$ satisfy condition (2.9). Hence we have

$$
f_{1}(-R)=w\left(a_{1}+\int_{(0, \infty)} \frac{\tau_{1}(d u)}{u+R}\right)=w\left(a_{1}+g_{1}(R)\right) .
$$

Note that $g_{1}(R) \rightarrow 0$ as $R \rightarrow \infty$ and, if $\tau_{1} \not \equiv 0$, then

$$
g_{1}(R) \geqslant \frac{\tau_{1}((0, R))}{R} \geqslant \frac{c}{R}
$$

for sufficiently large $R \geqslant 1$. Here and in the sequel we shall denote by $c$ positive constants which do not depend on $R$. In view of (5.3), we obtain the formula

$$
f_{2}(-R)=a_{2}+\int_{(0, \infty)} \frac{\tau_{2}(d u)}{u-w\left(a_{1}+g_{1}(R)\right)}=a_{2}+g_{2}\left(-w\left(a_{1}+g_{1}(R)\right)\right)
$$


If in (5.5) $a_{1}>0$, then, it is easy to see, that $\left|f_{2}(-R)\right| \leqslant c$ and we arrive at (5.2). Let $a_{1}=0$. In this case we need an upper bound for the function $\left|g_{2}\left(-w g_{1}(R)\right)\right|$ for large $R \geqslant 1$.

Since $a_{1}=0$, we conclude $\tau_{1} \not \equiv 0$ and write, using (5.4),

$$
\begin{aligned}
& \operatorname{Im} g_{2}\left(-w g_{1}(R)\right)=\int_{(0, \infty)} \frac{g_{1}(R) \operatorname{Im} w}{\left(u-g_{1}(R) \operatorname{Re} w\right)^{2}+\left(g_{1}(R) \operatorname{Im} w\right)^{2}} \tau_{2}(d u) \\
& \leqslant \frac{\tau_{2}\left(\left(0,-g_{1}(R) \log g_{1}(R)\right)\right)}{g_{1}(R) \operatorname{Im} w}+c g_{1}(R) \int_{\left[-g_{1}(R) \log g_{1}(R), \infty\right)} \frac{\tau_{2}(d u)}{u^{2}} \\
& \leqslant c\left(\frac{\tau_{2}\left(\left(0,-g_{1}(R) \log g_{1}(R)\right)\right)}{g_{1}(R)}+\frac{1}{g_{1}(R)\left(\log g_{1}(R)\right)^{2}}\right)=o\left(\frac{1}{g_{1}(R)}\right)=o(R)
\end{aligned}
$$

as $R \rightarrow \infty$. In addition we have

$$
\begin{aligned}
& \left|\operatorname{Re} g_{2}\left(-w g_{1}(R)\right)\right|=\left|\int_{(0, \infty)} \frac{\left(u-g_{1}(R) \operatorname{Re} w\right)}{\left(u-g_{1}(R) \operatorname{Re} w\right)^{2}+\left(g_{1}(R) \operatorname{Im} w\right)^{2}} \tau_{2}(d u)\right| \\
& \leqslant c\left(\frac{\tau_{2}\left(\left(0,2|w| g_{1}(R)\right)\right)}{g_{1}(R)}+\int_{\left[-g_{1}(R) \log g_{1}(R), \infty\right)} \frac{\tau_{2}(d u)}{u}\right. \\
& \left.+\int_{\left[2|w| g_{1}(R),-g_{1}(R) \log g_{1}(R)\right)} \frac{\tau_{2}(d u)}{u}\right)=o\left(\frac{1}{g_{1}(R)}\right)=o(R), \quad R \rightarrow \infty .
\end{aligned}
$$

The estimate (5.2) and, hence (5.1), follows immediately from (5.6) and (5.7).

Now we shall prove that

$$
R \max _{z \in \gamma_{2,4}}\left|f_{2}(z)\right| \rightarrow \infty, \quad R \rightarrow \infty .
$$

We shall write $f_{1}(z)$ in the form

$$
\begin{aligned}
f_{1}(z) & =w\left(\operatorname{Re}\left(R_{\mu_{1}}(z) / z\right)+i \operatorname{Im}\left(R_{\mu_{1}}(z) / z\right)\right) \\
& =w\left(a_{1}+\int_{(0, \infty)} \frac{(u-\operatorname{Re} z)}{(u-\operatorname{Re} z)^{2}+(\operatorname{Im} z)^{2}} \tau_{1}(d u)\right. \\
& \left.+i \int_{(0, \infty)} \frac{\operatorname{Im} z}{(u-\operatorname{Re} z)^{2}+(\operatorname{Im} z)^{2}} \tau_{1}(d u)\right) .
\end{aligned}
$$

Let $|z|=1 / R$ and $\eta|\operatorname{Re} z| \leqslant \operatorname{Im} z$, where $\eta:=\min \{\tan \alpha, 1 / 10\}$. For these $z$, using (5.9), we obtain for sufficiently large $R \geqslant 1$ the inequality

$$
\left|\operatorname{Re} \frac{R_{\mu_{1}}(z)}{z}\right| \leqslant a_{1}+\frac{\tau_{1}((0,2 \operatorname{Im} z / \eta))}{\operatorname{Im} z}+2 \int_{[2 \operatorname{Im} z / \eta, \infty)} \frac{\tau_{1}(d u)}{u}=o(R) .
$$


On the other hand the following lower bound holds

$$
\left|\operatorname{Re} \frac{R_{\mu_{1}}(z)}{z}\right| \geqslant a_{1}+\frac{1}{2} \int_{[2 \operatorname{Im} z / \eta, \infty)} \frac{\tau_{1}(d u)}{u}-\frac{\tau_{1}((0,2 \operatorname{Im} z / \eta))}{\operatorname{Im} z} .
$$

In addition, using (5.9), we deduce, for the same $z$,

$$
\frac{\eta^{2}}{10} q_{1}(z) \leqslant \operatorname{Im} \frac{R_{\mu_{1}}(z)}{z} \leqslant 4 q_{1}(z),
$$

where

$$
q_{1}(z):=\frac{\tau_{1}((0,2 \operatorname{Im} z / \eta))}{\operatorname{Im} z}+\operatorname{Im} z \int_{[2 \operatorname{Im} z / \eta, \infty)} \frac{\tau_{1}(d u)}{u^{2}}=o(R) .
$$

Comparing (5.11) and the left-hand side of inequality (5.12), we conclude that $\left|R_{\mu_{1}}(z) / z\right| \geqslant c$ for some positive constant $c$ and the $z$ considered above. From (5.10) and the right-hand side of inequality (5.12) we see that $\left|f_{1}(z)\right|=o(R)$ for those $z$. Moreover by (5.3) and by the definition of $f_{1}(z)$, it follows that $\arg w \leqslant$ $\arg f_{1}(z) \leqslant \pi+\arg w$ for $z \in \mathbb{C}^{+}$. Hence, we get, for $|z|=1 / R, \eta|\operatorname{Re} z| \leqslant \operatorname{Im} z$,

$$
c \leqslant\left|f_{1}(z)\right|=o(R) \text { and } \arg w \leqslant \arg f_{1}(z) \leqslant \pi+\arg w,
$$

with some positive constant $c$.

Let $|z|=1 / R$ and $\operatorname{Re} z \leqslant 0,-\eta \operatorname{Re} z>\operatorname{Im} z$. Repeating the previous arguments we obtain estimate (5.14) for such $z$. Thus, (5.14) holds for all $z \in \gamma_{2,4}$.

Using (5.3), it is not difficult to conclude that $\left|R_{\mu_{2}}(z)\right| \geqslant c\left(\delta_{1}, \delta_{2}\right) /|z|$ for $|z| \geqslant$ $\delta_{1}$ and $\delta_{2} \leqslant \arg z \leqslant 2 \pi-\delta_{2}$, where $\delta_{1}>0, \delta_{2} \in(0, \pi)$ are constants and $c\left(\delta_{1}, \delta_{2}\right)$ is a positive constant depending on $\delta_{1}$ and $\delta_{2}$. Using this estimate, we arrive at the lower bound

$$
\left|\frac{R_{\mu_{2}}\left(f_{1}(z)\right)}{f_{1}(z)}\right| \geqslant \frac{c}{\left|f_{1}(z)\right|} \geqslant \frac{N(R)}{R}, \quad z \in \gamma_{2,4},
$$

where $N(R) \rightarrow \infty$ as $R \rightarrow \infty$. Relation (5.8) follows from this bound.

Now we let $z$ run through $\gamma_{2}$ in the counter clockwise direction.

We see from the arguments of the beginning of the proof of the lemma that $\alpha-\arg w<\arg f_{2}(z)<\pi-\arg w$ for $z \in \mathbb{C}$ such that $\alpha \leqslant \arg z<\pi$. If $z$ traverses $\gamma_{2,1}$, the image $\zeta=f_{3}(z)$ lies in the angular region $\arg w+\alpha-\pi<\arg \zeta<\arg w$ in the $\zeta$-plane.

If $z$ traverses $\gamma_{2,2}$, by (5.1), the image $\zeta=f_{3}(z)$ lies in the domain $|\zeta|>$ $N_{1}(R), \arg w+\alpha-\pi \leqslant \arg \zeta \leqslant \pi$, where $N_{1}(R) \rightarrow \infty$ as $R \rightarrow \infty$.

If $z$ traverses $\gamma_{2,3}$, the image $\zeta=f_{3}(z)$ lies in the angular domain $\arg w<$ $\arg \zeta \leqslant \pi$.

Finally, by (5.8), when $z$ moves in $\gamma_{2,4}$, the image $\zeta=f_{3}(z)$ lies in the disk $|\zeta|<1 / N_{2}(R)$, where $N_{2}(R) \rightarrow \infty$ as $R \rightarrow \infty$.

In view of these results about the image $\zeta=f_{3}(z)$, we see that the winding number of $f_{3}(z)$ around $w$, when $z$ traverses $\gamma_{2}$ in the counter clockwise direction, 
is equal to one. Hence, by the argument principle, the function $f_{3}(z)$ takes the value $w$ precisely once inside $\gamma_{2}$. Since this assertion holds for all sufficiently large $R>1$ and all $0<\alpha<\arg w, f_{3}(z)$ takes the value $w$ precisely once in $\mathbb{C}^{+}$. In addition, it takes this value in the domain $\arg w \leqslant \arg z<\pi$. This proves the lemma.

Lemma 5.2. For every $z \in \mathbb{C}^{+}$there exist unique points $z_{1} \in \mathbb{C}^{+}$and $z_{2} \in \mathbb{C}^{+}$ such that $z_{1} z^{-1} \in \mathbb{C}^{+} \cup(0, \infty)$ and $z_{2} z^{-1} \in \mathbb{C}^{+} \cup(0, \infty)$, and

$$
z_{1} z_{2}=z R_{\mu_{1}}\left(z_{1}\right) \quad \text { and } \quad z_{1} z_{2}=z R_{\mu_{2}}\left(z_{2}\right) \text {. }
$$

Proof. Fix $z \in \mathbb{C}^{+}$. In view of the second relation of (5.15), we have $z_{1}=$ $z R_{\mu_{2}}\left(z_{2}\right) / z_{2}$. Since $R_{\mu_{2}}\left(z_{2}\right)$ belongs to the class $\mathcal{K}$, we obtain $z_{1} \in \mathbb{C}^{+}$and $z_{1} z^{-1} \in$ $\mathbb{C}^{+} \cup(0, \infty)$ provided that $z_{2} \in \mathbb{C}^{+}$and $z_{2} z^{-1} \in \mathbb{C}^{+} \cup(0, \infty)$. It remains to solve the functional equation

$$
R_{\mu_{2}}\left(z_{2}\right)=R_{\mu_{1}}\left(z R_{\mu_{2}}\left(z_{2}\right) / z_{2}\right) .
$$

Rewrite it in the form $z=z_{2} / R\left(z_{2} ; z\right)$, where

$$
R\left(z_{2} ; z\right):=R_{\mu_{1}}\left(z R_{\mu_{2}}\left(z_{2}\right) / z_{2}\right) /\left(z R_{\mu_{2}}\left(z_{2}\right) / z_{2}\right)=R_{\mu_{1}}\left(z_{1}\right) / z_{1} .
$$

We see that, for fixed $z \in \mathbb{C}^{+}$, the function $\tilde{f}_{3}\left(z_{2}\right):=z_{2} / R\left(z_{2} ; z\right)$ has the same type as the function $f_{3}$ in Lemma 5.1. Applying Lemma 5.1 to the function $\tilde{f}_{3}$, we obtain the assertion of the lemma.

We need as well the following auxiliary lemma which is an analogue of Lemma 4.1. Denote $\mathbb{S}_{\pi}:=\{z \in \mathbb{C}: 0<\operatorname{Im} z<\pi\}$.

Lemma 5.3. Assume that $f_{4}$ has the representation

$$
f_{4}(z):=-a_{1} z+\frac{a_{2}}{z}+\int_{(0, \infty)} \frac{1+u z}{z-u} \sigma(d u), \quad 0<\arg z<2 \pi,
$$

where $a_{j} \geqslant 0, j=1,2$, and $\sigma$ is a finite nonnegative measure. Then the function $f_{5}(z):=\log z+f_{4}(z), f_{5}: \mathbb{C}^{+} \rightarrow \mathbb{C}$ takes every value in $\mathbb{S}_{\pi}$ precisely once. The inverse function $f_{5}^{(-1)}: \mathbb{S}_{\pi} \rightarrow \mathbb{C}^{+}$thus defined has the property that $f_{5}^{(-1)}(\log z)$ belongs to the class $\mathcal{K}$.

Proof. We shall prove that for every $w \in \mathbb{S}_{\pi}$ there exists an unique $z \in \mathbb{C}^{+}$such that

$$
w=f_{5}(z)=\log z+f_{4}(z) .
$$

Recall that we take the principle branch of the logarithm only. Let $\alpha \in(0, \operatorname{Im} w)$ and let $\gamma_{2}$ be the curve defined in the proof of Lemma 5.1.

Let $z$ traverse $\gamma_{2,3}$. The image $\zeta=f_{5}(z)$ lies on the line $\operatorname{Im} \zeta=\pi$. Furthermore, we have $\log (-R)+f_{4}(-R)=\log R+f_{4}(-R)+i \pi$ with $\log R+f_{4}(-R) \geqslant(\log R) / 2$, and $\log (-1 / R)+f_{4}(-1 / R)=-\log R+f_{4}(-1 / R)+i \pi$ with $-\log R+f_{4}(-1 / R) \leqslant$ $-(\log R) / 2$. 
Let $z$ move in $\gamma_{2,4}$. Since

$$
\int_{(0, \infty)} \frac{u|z|}{|z-u|} \sigma(d u) \leqslant \sigma((0, \infty))|z| d(z), \quad z \in \mathbb{C}^{+}
$$

where $d(z):=1$ for $\operatorname{Re} z \leqslant 0$ and $d(z):=|z| / \operatorname{Im} z$, for $\operatorname{Re} z>0$, we easily conclude, for $z \in \gamma_{2,4}$, Re $z \leqslant 0$,

$$
\operatorname{Re}\left(f_{4}(z)+a_{1} z-\frac{a_{2}}{z}\right) \leqslant \frac{c}{R}, \quad R \rightarrow \infty,
$$

with some positive constant $c$, and, for $z \in \gamma_{2,4}$, Re $z>0$,

$$
\arg \left(f_{4}(z)+a_{1} z-\frac{a_{2}}{z}\right)=\arg \left(-\int_{(0, \infty)} \frac{u \sigma(d u)}{|z-u|^{2}}+\bar{z} \int_{(0, \infty)} \frac{\sigma(d u)}{|z-u|^{2}}+O(1 / R)\right) \leqslant 2 \pi-\frac{\alpha}{2} \text {. }
$$

Hence the image $\zeta=f_{5}(z)$ either lies in the domain $\{\zeta \in \mathbb{C}: \alpha<\operatorname{Im} \zeta<\pi,|\zeta| \geqslant$ $c(\alpha) \log R\}$, where $c(\alpha)$ is a positive constant, or in the half-plane $\{\zeta \in \mathbb{C}: \operatorname{Im} \zeta \leqslant$ $\alpha\}$.

Let $z$ traverse $\gamma_{2,1}$. Note that the image $\zeta=f_{5}(z)$ lies in the half-plane $\operatorname{Im} \zeta \leqslant$ $\alpha$.

Let finally $z$ traverse $\gamma_{2,2}$. Since $\operatorname{Im} f_{4}(z) \leqslant 0$, we see that the image $\zeta=f_{5}(z)$ lies in the half-plane $\{\zeta \in \mathbb{C}: \operatorname{Im} \zeta \leqslant \alpha\}$ or in the domain $\{\zeta \in \mathbb{C}: \alpha<\operatorname{Im} \zeta<\pi\}$.

Assume that $\zeta=f_{5}(z)$ lies in $\{\zeta \in \mathbb{C}: \alpha<\operatorname{Im} \zeta<\pi\}$. In this case we easily see that $-\operatorname{Im} f_{4}(z) \leqslant \pi-\alpha$ and we obtain the inequality

$$
-\operatorname{Im} f_{4}(z)=-a_{1} \operatorname{Im} z+a_{2} \frac{\operatorname{Im} z}{|z|^{2}}+\operatorname{Im} z \int_{(0, \infty)} \frac{1+u^{2}}{|z-u|^{2}} \sigma(d u) \leqslant \pi-\alpha,
$$

On the other hand note that, for $z \in \gamma_{2,2}$,

$$
\operatorname{Re} f_{4}(z)=-a_{1} \operatorname{Re} z+\int_{(0, \infty)} \frac{u\left(|z|^{2}-u \operatorname{Re} z\right)}{|z-u|^{2}} \sigma(d u)+O(1), \quad R \rightarrow \infty .
$$

Since, for $z \in \gamma_{2,2},\left|f_{4}(z)+a_{1} z-a_{2} / z\right|=o(R)$, we deduce from (5.21) in the case $a_{1} \neq 0$ the bound $\left|\operatorname{Re} f_{4}(z)\right| \geqslant c R$. This means that $\zeta=f_{5}(z)$ lies in the domain $\left\{\zeta \in \mathbb{C}: \alpha<\operatorname{Im} \zeta<\pi,|\zeta|>\frac{1}{2} \log R\right\}$. If $a_{1}=0$, then we have from (5.20) and (5.21)

$$
\begin{aligned}
\operatorname{Re} f_{4}(z) & \geqslant \int_{(0, \infty)} \frac{u R^{2}}{|z-u|^{2}} \sigma(d u)-(1+\operatorname{sign}(\operatorname{Re} z)) \frac{\operatorname{Re} z}{\operatorname{Im} z}(\pi-\alpha)+O(1) \\
& \geqslant R^{2} \int_{(0, \infty)} \frac{u \sigma(d u)}{|z-u|^{2}}+O(1), \quad R \rightarrow \infty
\end{aligned}
$$

We conclude again that $\zeta=f_{5}(z)$ lies in the domain $\{\zeta \in \mathbb{C}: \alpha<\operatorname{Im} \zeta<\pi,|\zeta|>$ $\left.\frac{1}{2} \log R\right\}$. 
Hence the image $\zeta=f_{5}(z)$ for $z \in \gamma_{2,2}$ lies in the half-plane $\{\zeta \in \mathbb{C}: \operatorname{Im} \zeta \leqslant \alpha\}$ or in the domain $\left\{\zeta \in \mathbb{C}: \alpha<\operatorname{Im} \zeta<\pi,|\zeta|>\frac{1}{2} \log R\right\}$.

Therefore we conclude that the image $\zeta=f_{5}(z)$ winds around $w$ once when $z$ runs through $\gamma_{2}$. By the argument principle, there is an unique point $z$ inside $\gamma_{2}$ such that (5.19) holds. This relation is valid for all sufficiently large $R>1$ and sufficiently small $\alpha>0$. Thus for every fixed $w \in \mathbb{S}_{\pi}$ there is an unique point $z \in \mathbb{C}^{+}$such that (5.19) holds. This implies that the inverse function $q=f_{5}^{(-1)}: \mathbb{S}_{\pi} \rightarrow \mathbb{C}^{+}$exists and is analytic on $\mathbb{S}_{\pi}$.

Let us show that $q(z)$ admits an analytic continuation on the half-line $\gamma_{-}$: $\operatorname{Im} z=\pi, \operatorname{Re} z<0$, and that its value on this half-line is negative. It is easy to see that

$$
f_{5}^{\prime}(x)=\frac{1}{x}-a_{1}-\frac{a_{2}}{x^{2}}-\int_{(0, \infty)} \frac{\left(1+u^{2}\right) \sigma(d u)}{(x-u)^{2}}<0, \quad x<0 .
$$

Since $f_{5}(z)$ is analytic on $(-\infty, 0)$, we conclude that $f_{5}^{(-1)}$ exists and is analytic on $\gamma_{-}$as well. Since, as shown above, for every fixed $w \in \mathbb{S}_{\pi}$ there is an unique point $z \in \mathbb{C}^{+}$such that (5.19) holds, this function coincides for $z \in \mathbb{C}^{+}$with the function $q(z)$ obtained early. Introduce the function $f_{6}(z):=q(\log z), z \in \mathbb{C}^{+}$. Note that $f_{6} \in \mathcal{N}$ and $f_{6}^{(-1)}(z)=z \exp \left\{f_{4}(z)\right\}$, on the domain $\mathbb{C}^{+}$, where $f_{6}^{(-1)}$ exists. Moreover, the function $f_{6}(z)$ admits an analytic continuation on $(-\infty, 0)$.

From the definition of $f_{6}(z)$ it follows that $f_{6}(x)<0$ for $x<0$ and $f_{6}(x) \rightarrow 0$ as $x \uparrow 0$. By Corollary 3.3, $f_{6}$ belongs to the class $\mathcal{K}$ and we obtain the assertion of the lemma.

Proof of Theorem 2.4. Consider the function

$$
F\left(z, z_{2}\right):=R_{\mu_{2}}\left(z_{2}\right)-R_{\mu_{1}}\left(z R_{\mu_{2}}\left(z_{2}\right) / z_{2}\right)
$$

which, by (2.8), is analytic on $\mathbb{C}^{+} \times \mathbb{C}^{+}$. By Lemma 5.2, for every fixed $z=z^{0} \in \mathbb{C}^{+}$ the equation (5.16) has an unique solution $z_{2}=z_{2}^{0} \in\left\{z_{2} \in \mathbb{C}: \arg z^{0} \leqslant \arg z_{2}<\right.$ $\pi\}$. Let us show that the function $F\left(z, z_{2}\right)$ satifies (3.10) at the point $\left(z^{0}, z_{2}^{0}\right)$. Note that if $R_{\mu_{2}}\left(z_{2}\right)=R_{\mu_{1}}\left(z^{0} R_{\mu_{2}}\left(z_{2}\right) / z_{2}\right)$ holds for $z_{2} \in \mathbb{C}^{+}$such that $\left|z_{2}-z_{2}^{0}\right|<r^{\prime}$ with $r^{\prime}>0$, then this equality holds for all $z_{2} \in \mathbb{C}^{+}$. By (5.2), the function $R\left(-r ; z^{0}\right)$ introduced in (5.17) has the property $R\left(-r ; z^{0}\right) / r \rightarrow 0$ as $r \rightarrow \infty$. Since the relation $z^{0}=-r / R\left(-r ; z^{0}\right)$ holds for $r>0$, we arrive at a contradiction. Hence the point $\left(z^{0}, z_{2}^{0}\right)$ satisfies the assumptions (3.10) of Theorem 3.7. Now repeating almost word for word the arguments of the proof of Theorem 2.1, using Lemma 5.2 instead of Lemma 4.2, and Theorem [3.1. Corollary 3.3, we obtain the assertion of the theorem.

We prove Corollaries 2.5 and 2.6 in the same way as Corollaries 2.2 and 2.3. The relation (2.12) with $n=t \in[1, \infty)$ follows from Lemmas 5.2 and 5.3 , repeating the arguments used in the proof of Theorem 2.1. 
2. Consider the case of multiplicative convolution for p-measures of the class $\mathcal{M}_{*}$. In order to prove Theorem 1.7 we need some auxiliary results.

Lemma 5.4. Let $Q \in \mathcal{S}$ and $Q(0) \neq 0$. Then the function $g: \mathbb{D} \backslash\{0\} \rightarrow \mathbb{C}: z \mapsto$ $Q(z) / z$ takes every value in $\mathbb{C} \backslash \overline{\mathbb{D}}$ precisely once.

Proof. Without loss of generality we assume that $Q(0)>0$. Let $w \in \mathbb{C} \backslash \overline{\mathbb{D}}$ and let $r \in(0,1)$. We assume that $1-r$ is sufficiently small. It is easy to see that there exist $\theta=\theta(w, r) \in[0,2 \pi)$ and $\theta \neq \arg w$ such that $Q(z) / z \neq w$ for $z=\rho e^{i \theta}, 1-r \leqslant \rho \leqslant r$. Let $\gamma_{3}=\gamma_{3}(w, \theta)$ (see Figure 3) be the closed rectifiable curve consisting of the two circles $\gamma_{3,2}:|z|=1-r$ and $\gamma_{3,4}:|z|=r$, and the segments $\gamma_{3,1}, \gamma_{3,3}$ which coincide with the segment $\gamma: t e^{i \theta}, 1-r \leqslant t \leqslant r$, traversed twice in opposite directions.

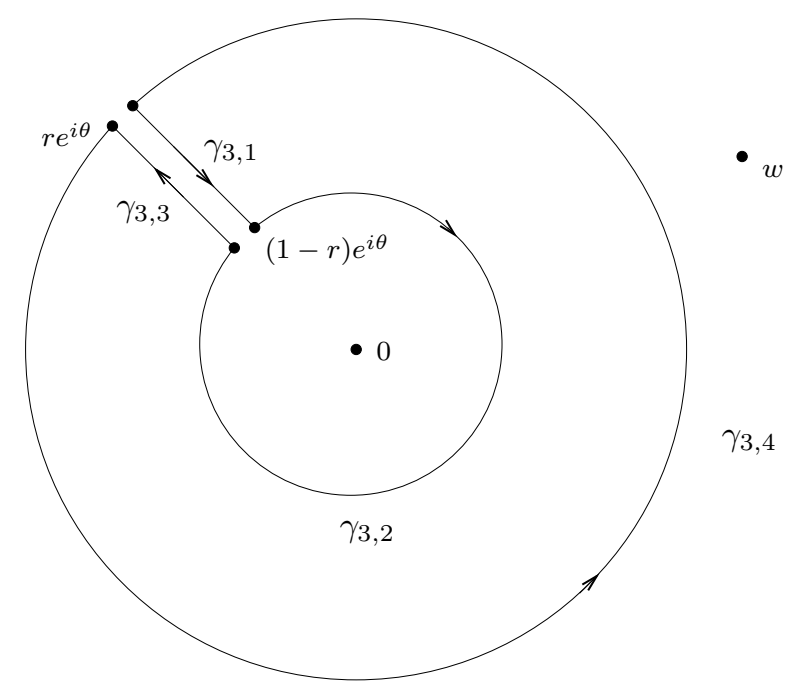

FiguRE 3.

We note that the number of loops around $w$ by the map $z \mapsto Q(z) / z$ as $z$ traverses the curve $\gamma_{3}$ once in the counter clockwise direction is equal to 1 . Indeed, if $z$ traverses $\gamma_{3,1}$ the number of windings around $w$ is equal to some number $\alpha$. If $z$ traverses $\gamma_{3,4}$ the number of windings around $w$ is equal to 0 . Finally, moving along $\gamma_{3,3}$, the number of windings around $w$ is equal to $-\alpha$. Hence traversing $\gamma_{3,1}, \gamma_{3,4}$ and $\gamma_{3,3}$ in the counter clockwise direction the number of windings around $w$ is equal to 0 . Traversing $\gamma_{3,2}$, we wind around $w$ once. Hence, by the argument principle, the function $Q(z) / z$ takes the value $w$ in the domain $\{z: 1-r<|z|<r\}$ precisely once. Since this assertion holds for all $0<r<1, Q(z) / z$ takes every value $w \in \mathbb{C} \backslash \overline{\mathbb{D}}$ in $\mathbb{D}$ precisely once.

Let $\mu_{1}$ and $\mu_{2}$ belong to the class $\mathcal{M}_{*}$. Denote $a_{j}:=\int_{\mathbb{T}} \xi \mu_{j}(d \xi), j=1,2$. Recall that $Q_{\mu_{j}}(0)=0, a_{j} \neq 0, j=1,2$. 
Lemma 5.5. For every $z \in \mathbb{D} \backslash\{0\}$, there exist unique points $z_{1} \in \mathbb{D}$ and $z_{2} \in \mathbb{D}$ such that $\left(z_{1}, z_{2}\right) \neq(0,0)$ and

$$
z_{2}=z \frac{Q_{\mu_{1}}\left(z_{1}\right)}{z_{1}} \quad \text { and } \quad z_{1}=z \frac{Q_{\mu_{2}}\left(z_{2}\right)}{z_{2}} .
$$

Proof. Fix $z \in \mathbb{D} \backslash\{0\}$. Assume for definiteness $\left|a_{2}\right| \leqslant\left|a_{1}\right|$. It follows from Proposition 3.5 that $Q_{\mu_{1}}\left(a_{2} z\right) \neq 0$ for every $z \in \mathbb{D}$.

By Schwarz's lemma, $\left|Q_{\mu_{2}}\left(z_{2}\right) / z_{2}\right| \leqslant 1, z_{2} \in \mathbb{D}$. The second relation of (5.22) implies that if $z_{2} \in \mathbb{D}$, then $z_{1} \in \mathbb{D}$ as well. Hence, by the first relation of (5.22), we need to solve the functional equation

$$
Q_{\mu_{2}}\left(z_{2}\right)=Q_{\mu_{1}}\left(z Q_{\mu_{2}}\left(z_{2}\right) / z_{2}\right), \quad z_{2} \in \mathbb{D} .
$$

Rewrite this relation in the form $z_{2} / z=Q\left(z_{2}\right)$, where

$$
Q\left(z_{2}\right)=Q_{\mu_{1}}\left(z Q_{\mu_{2}}\left(z_{2}\right) / z_{2}\right) /\left(z Q_{\mu_{2}}\left(z_{2}\right) / z_{2}\right) .
$$

The function $Q$ satisfies $Q(0)=Q_{\mu_{1}}\left(a_{2} z\right) /\left(a_{2} z\right) \neq 0$ and $Q \in \mathcal{S}$. By Lemma 5.4, we obtain the assertion of the lemma for all $z \in \mathbb{D} \backslash\{0\}$.

We need as well the following auxiliary lemma which is an analogue of Lemma 4.1 for the semigroup $\left(\mathcal{M}_{*}, \otimes\right)$.

Lemma 5.6. Define

$$
Q_{1}(z):=\int_{\mathbb{T}} \frac{\xi+z}{\xi-z} \sigma(d u), \quad z \in \mathbb{D},
$$

where $\sigma$ is finite nonnegative measure. Then the function $Q_{2}: \mathbb{D} \rightarrow \mathbb{C}, Q_{2}(z):=$ $z \exp \left\{Q_{1}(z)\right\}$, takes every value in $\mathbb{D}$ precisely once. The inverse $Q_{2}^{(-1)}: \mathbb{D} \rightarrow \mathbb{D}$ thus defined is in the class $\mathcal{S}_{*}$.

Proof. Let $w \in \mathbb{D}$. Introduce the curve $\gamma_{4}:|z|=r$, where $r \in(0,1)$ and $1-r$ is sufficiently small. Note that $\operatorname{Im} Q_{1}(0)=0$. Then, by the minimum and maximum principles for harmonic functions, for every $r \in(0,1)$ there exist points $\theta^{\prime}$ and $\theta^{\prime \prime} \in[0,2 \pi)$ such that $\operatorname{Im} Q_{1}\left(r e^{i \theta^{\prime}}\right)<0$ and $\operatorname{Im} Q_{1}\left(r e^{i \theta^{\prime \prime}}\right)>0$. Since for every $r \in(0,1) \theta \mapsto \operatorname{Im} Q_{1}\left(r e^{i \theta}\right)$ is analytic on the segment $[0,2 \pi]$, there are only a finite number of points $\left\{r e^{i \theta_{l}}\right\}_{l=1}^{k}$ with $0 \leqslant \theta_{1}<\theta_{2}<\cdots<\theta_{k}<2 \pi$, such that $\operatorname{Im} Q_{1}\left(r e^{i \theta_{l}}\right)=0, l=1,2, \ldots, k$. Consider the $\operatorname{arcs} \gamma_{4, l}: \theta_{l}<\arg z \leqslant \theta_{l+1},|z|=r$, $l=1, \ldots, k$, with $\theta_{k+1}=\theta_{1}+2 \pi$.

Let $z$ run transverse $\gamma_{4, l}$ in the counter clockwise direction. Since $\left|\exp \left\{Q_{1}(z)\right\}\right| \geqslant$ 1, we note that the change in $\operatorname{Arg}\left(Q_{2}(z)-w\right)$ is equals to

$$
\left(\arg \left(r e^{i \theta_{l+1}}-w\right)-\arg \left(r e^{i \theta_{l}}-w\right)\right) /(2 \pi) .
$$

Hence the image $\zeta=Q_{2}(z)$ winds around $w$ once when $z$ transverses $\gamma_{4}$ once in the counter clockwise direction. By the argument principle, the function $Q_{2}: \mathbb{D} \rightarrow$ $\mathbb{C}$ takes the value $w \in \mathbb{D}$ in $\mathbb{D}$ precisely once. The inverse function $Q_{2}^{(-1)}: \mathbb{D} \rightarrow \mathbb{D}$ thus defined is analytic on $\mathbb{D}$ and obviously belongs to the class $\mathcal{S}_{*}$. The lemma is proved. 


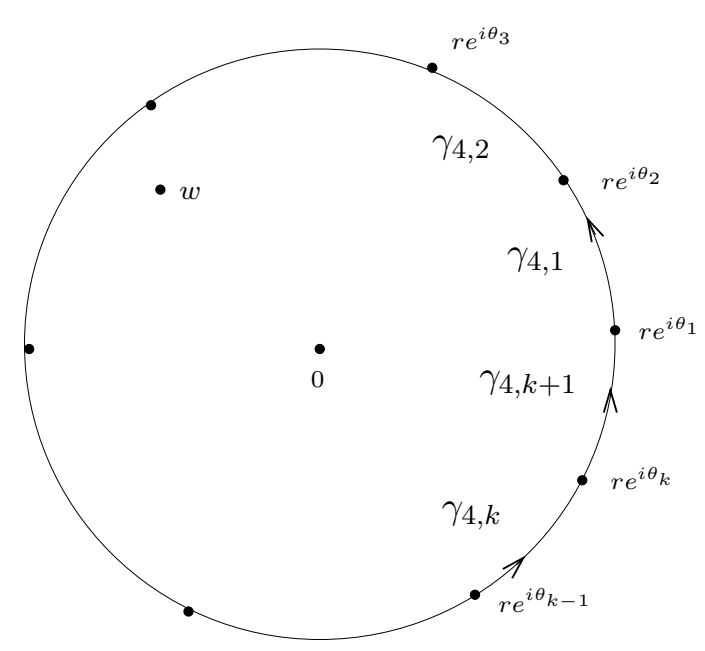

FIGURE 4.

Proof of Theorem 2.7. We assume for definiteness that $\left|a_{2}\right| \leqslant\left|a_{1}\right|$. Consider the function $F\left(z, z_{2}\right):=Q_{\mu_{2}}\left(z_{2}\right)-Q_{\mu_{1}}\left(z Q_{\mu_{2}}\left(z_{2}\right) / z_{2}\right)$ which is analytic on $\mathbb{D} \times$ $\mathbb{D}$. For a fixed $z=z^{0} \in \mathbb{D}$ equation (5.23) has, by Lemma 5.5, an unique solution $z_{2}=z_{2}^{0} \in \mathbb{D}$. Moreover, if $F\left(z^{0}, z_{2}\right)=0$ for $z_{2}$ in some neighborhood of the point $z_{2}^{0}$, then $F\left(z^{0}, z_{2}\right)=0$ for all $z_{2} \in \mathbb{D}$. This relation is equivalent to $z_{2} / z^{0}=Q\left(z_{2}\right), z_{2} \in \mathbb{D}$, where $Q \in \mathcal{S}$. Choosing here $\left|z_{2}\right|>\left|z^{0}\right|$ we arrive at a contradiction. Hence the function $F\left(z, z_{2}\right)$ satisfies the assumptions (3.10) of Theorem 3.7 at the point $\left(z^{0}, z_{2}^{0}\right)$. Repeating almost word for word the arguments of the proof of Theorem 2.1, using Lemma 5.5 instead of Lemma 4.2, we obtain that there exists a neighborhood $\left|z-z^{0}\right|<r\left(z^{0}\right)$ such that the equation $F\left(z, z_{2}\right)=$ 0 has an unique regular solution $z_{2}=z_{2}\left(z ; z^{0}\right)$ with values in $\mathbb{D}$. Choosing $z_{1}\left(z ; z^{0}\right):=z Q_{\mu_{2}}\left(z_{2}\left(z ; z^{0}\right)\right) / z_{2}\left(z ; z^{0}\right)$, there are two regular functions $z_{1}\left(z ; z^{0}\right)$ and $z_{2}\left(z ; z^{0}\right)$ with values in $\mathbb{D}$ which satisfy the equations

$$
z_{1}\left(z ; z^{0}\right) z_{2}\left(z ; z^{0}\right)=z Q_{\mu_{1}}\left(z_{1}\left(z ; z^{0}\right)\right) \quad \text { and } \quad z_{1}\left(z ; z^{0}\right) z_{2}\left(z ; z^{0}\right)=z Q_{\mu_{2}}\left(z_{2}\left(z ; z^{0}\right)\right)
$$

By Lemma 5.5, for the points $z^{\prime} \in \mathbb{D}$ and $z^{\prime \prime} \in \mathbb{D}, z^{\prime} \neq z^{\prime \prime}$, we obtain the identities $z_{1}\left(z ; z^{\prime}\right)=z_{1}\left(z ; z^{\prime \prime}\right), z_{2}\left(z ; z^{\prime}\right)=z_{2}\left(z ; z^{\prime \prime}\right)$ for $z$ of the domain $\left\{\left|z-z^{\prime}\right|<r\left(z^{\prime}\right)\right\} \cap$ $\left\{\left|z-z^{\prime \prime}\right|<r\left(z^{\prime \prime}\right)\right\}$. By the monodromy theorem, there exist regular functions $Z_{1}(z)$ and $Z_{2}(z), z \in \mathbb{D}$, such that, for every point $z^{0} \in \mathbb{D}, Z_{j}(z)=z_{j}\left(z ; z^{0}\right), j=$ 1,2 , for $\left|z-z^{0}\right|<r\left(z^{0}\right)$. These functions belong to the class $\mathcal{S}$ and are unique solutions of equations (2.14) for $\mu_{j}, j=1,2$. Moreover, it is easy to see that $Z_{1}, Z_{2} \in \mathcal{S}_{*}$. This proves the theorem.

We prove Corollaries 2.8 and 2.9 in the same way as Corollaries 2.2 and 2.3. The relation (2.16) with $n=t \in[1, \infty)$ we obtain from Lemmas 5.5, and 5.6 , repeating the arguments which we employed in the proof of Theorem 2.1. 


\section{BASIC ARITHMETIC AND KHINTCHINE'S LIMIT THEOREMS FOR THE SEMIGROUPS $(\mathbf{M}, \circ)$}

In this section we shall show that the main theorems for Delphic semigroups which are formulated in Section 3 hold for the semigroups $(\mathbf{M}, \circ)$. This proves Therems 2.11, and 2.12. We prove Khintchine's limit theorem for triangular arrays (Theorem 2.10) as well. At the end of this section we give a new proof for the integral representations of the Voiculescu transforms of i.d. elements of the semigroups $(\mathbf{M}, \circ)$.

Introduce sets $\mathbf{M}^{\gamma}$ of measures of $\mathbf{M}$ in the following way.

Consider the semigroup $(\mathcal{M}, \boxplus)$. Let $\bar{\Gamma}_{\alpha, \beta}$ denote the closure of $\Gamma_{\alpha, \beta}$. Denote by $\mathcal{M}^{(\alpha, \beta)}$ the set of those $\mu \in \mathcal{M}$ such that $F_{\mu}^{\prime}(z) \neq 0$ when $z \in \Omega_{\mu}$, where $\Omega_{\mu} \subseteq \mathbb{C}^{+}$is a domain, which $F_{\mu}\left(\Omega_{\mu}\right) \supset \bar{\Gamma}_{\alpha, \beta}$. The function $F_{\mu}(z)$ has an inverse $F_{\mu}^{(-1)}(z)$ defined on $\bar{\Gamma}_{\alpha, \beta}$ such that $\operatorname{Im} \varphi_{\mu}(z) \leqslant 0$. We denote $\mathbf{M}^{\gamma}:=\mathcal{M}^{(\alpha, \beta)}$, where $\gamma:=(\alpha, \beta) \in \mathcal{G}:=\{(\alpha, \beta): \alpha>0, \beta>0\}$, a subset of the semigroup $(\mathcal{M}, \boxplus)$.

Consider $\left(\mathcal{M}_{+}, \otimes\right)$ and let $\bar{\Gamma}_{\alpha, \beta, \Delta}^{+}$denote the closure of $\Gamma_{\alpha, \beta, \Delta}^{+}$. Denote by $\mathcal{M}_{+}^{(\alpha, \beta, \Delta)}$ a subset of the set of those $\mu \in \mathcal{M}_{+}$such that $R_{\mu}^{\prime}(z) \neq 0$ for $z \in \Omega_{\mu}$, where $\Omega_{\mu} \subseteq \mathbb{C} \backslash[0,+\infty)$ is a domain, with $R_{\mu}\left(\Omega_{\mu}\right) \supset \bar{\Gamma}_{\alpha, \beta, \Delta}^{+}$. The function $R_{\mu}(z)$ has an inverse $R_{\mu}^{(-1)}(z)$ defined on $\bar{\Gamma}_{\alpha, \beta, \Delta}^{+}$such that $\arg \Sigma_{\mu}(z) \leqslant 0$ for $z \in \bar{\Gamma}_{\alpha, \beta, \Delta}^{+} \cap \mathbb{C}^{+}$and $\arg \Sigma_{\mu}(z) \geqslant 0$ for $z \in \bar{\Gamma}_{\alpha, \beta}^{+} \cap \mathbb{C}^{-}$. As above we write $\mathbf{M}^{\gamma}:=$ $\mathcal{M}_{+}^{(\alpha, \beta, \Delta)}$, where $\gamma:=(\alpha, \beta, \Delta) \in \mathcal{G}:=\{(\alpha, \beta, \Delta): \alpha \in(0, \pi), 0<\beta<\Delta<\infty\}$, for a subset of the semigroup $\left(\mathcal{M}_{+}, \otimes\right)$.

Consider $\left(\mathcal{M}_{*}, \otimes\right)$ and let $\overline{\mathbb{D}}_{\alpha}$ denote the closure of $\mathbb{D}_{\alpha}:=\{z \in \mathbb{C}:|z|<\alpha\}$. Denote by $\mathcal{M}_{*}^{\alpha}$ the set of those $\mu \in \mathcal{M}_{*}$ such that $Q_{\mu}^{\prime}(z) \neq 0$ as $z \in \Omega_{\mu}$, where $\Omega_{\mu} \subseteq \mathbb{D}$ is a domain, with $Q_{\mu}\left(\Omega_{\mu}\right) \supset \overline{\mathbb{D}}_{\alpha}$. The function $Q_{\mu}(z)$ has an inverse $Q_{\mu}^{(-1)}(z)$ defined on $\overline{\mathbb{D}}_{\alpha}$ such that $\left|\Sigma_{\mu}(z)\right| \geqslant 1$. Introduce $\mathbf{M}^{\gamma}:=\mathcal{M}_{*}^{\alpha}$, where $\gamma:=\alpha \in \mathcal{G}:=\{\alpha: 0<\alpha<1\}$, for a subset of the semigroup $\left(\mathcal{M}_{*}, \nabla\right)$.

Proposition 6.1. For every fixed $\gamma \in \mathcal{G}, \mathbf{M}^{\gamma}$ is a semigroup with respect to the operation 0 .

Proof. At first we shall prove the assertion of the proposition for $\mathcal{M}^{(\alpha, \beta)}$. Let $\mu_{1}, \mu_{2} \in \mathcal{M}^{(\alpha, \beta)}$. It is sufficient to show that $\mu=\mu_{1} \boxplus \mu_{2} \in \mathcal{M}^{(\alpha, \beta)}$. By Theorem 2.1, there exist functions $Z_{1}, Z_{2} \in \mathcal{F}$ such that

$F_{\mu}(z)=F_{\mu_{1}}\left(Z_{1}(z)\right)=F_{\mu_{2}}\left(Z_{2}(z)\right) \quad$ and $\quad z=Z_{1}(z)+Z_{2}(z)-F_{\mu_{2}}\left(Z_{2}(z)\right), \quad z \in \mathbb{C}^{+}$.

By the definition of $\mathcal{M}^{(\alpha, \beta)}$, there exist domains $\Omega_{\mu_{j}}, j=1,2$, such that $F_{\mu_{j}}^{\prime}(z) \neq 0$ as $z \in \Omega_{\mu_{j}}$ and $F_{\mu_{j}}\left(\Omega_{\mu_{j}}\right) \supset \bar{\Gamma}_{\alpha, \beta}$. Consider the intersection of inverse images with respect to $Z_{j}, j=1,2, \Omega_{\mu}:=Z_{1}^{(-1)}\left(\Omega_{\mu_{1}}\right) \cap Z_{2}^{(-1)}\left(\Omega_{\mu_{2}}\right)$. Relations (6.1) imply $F_{\mu}^{\prime}(z)=F_{\mu_{1}}^{\prime}\left(Z_{1}(z)\right) Z_{1}^{\prime}(z)=F_{\mu_{2}}^{\prime}\left(Z_{2}(z)\right) Z_{2}^{\prime}(z), \quad 1=Z_{1}^{\prime}(z)+Z_{2}^{\prime}(z)\left(1-F_{\mu_{2}}^{\prime}\left(Z_{2}(z)\right)\right)$ 
as $z \in \mathbb{C}^{+}$. Hence $F_{\mu}^{\prime}(z) \neq 0$ for $z \in \Omega_{\mu}$. In addition note that $F_{\mu}\left(\Omega_{\mu}\right)=$ $F_{\mu_{1}}\left(\Omega_{\mu_{1}}\right) \cap F_{\mu_{2}}\left(\Omega_{\mu_{2}}\right) \supset \bar{\Gamma}_{\alpha, \beta}$, thus proving the assertion.

Consider $\mathcal{M}_{+}^{(\alpha, \beta, \Delta)}$. Let $\mu_{1}, \mu_{2} \in \mathcal{M}_{+}^{(\alpha, \beta, \Delta)}$. Let us show that $\mu=\mu_{1} \otimes \mu_{2} \in$ $\mathcal{M}_{+}^{(\alpha, \beta, \Delta)}$. By Theorem 1.4, there exist functions $Z_{1}, Z_{2} \in \mathcal{K}$ such that

$$
R_{\mu}(z)=R_{\mu_{1}}\left(Z_{1}(z)\right)=R_{\mu_{2}}\left(Z_{2}(z)\right) \quad \text { and } \quad Z_{1}(z) Z_{2}(z)=z R_{\mu_{2}}\left(Z_{2}(z)\right), \quad z \in \mathbb{C}^{+} .
$$

By the definition of $\mathcal{M}^{(\alpha, \beta, \Delta)}$, there exist domains $\Omega_{\mu_{j}}, j=1,2$, such that $R_{\mu_{j}}^{\prime}(z) \neq$ 0 as $z \in \Omega_{\mu_{j}}$ and $R_{\mu_{j}}\left(\Omega_{\mu_{j}}\right) \supset \bar{\Gamma}_{\alpha, \beta, \Delta}$. Denote $\Omega_{\mu}:=Z_{1}^{(-1)}\left(\Omega_{\mu_{1}}\right) \cap Z_{2}^{(-1)}\left(\Omega_{\mu_{2}}\right)$. Relations (6.2) imply

$$
\begin{aligned}
& R_{\mu}^{\prime}(z)=R_{\mu_{1}}^{\prime}\left(Z_{1}(z)\right) Z_{1}^{\prime}(z)=R_{\mu_{2}}^{\prime}\left(Z_{2}(z)\right) Z_{2}^{\prime}(z), \\
& Z_{1}^{\prime}(z) Z_{2}(z)+Z_{2}^{\prime}(z)\left(1-z R_{\mu_{2}}^{\prime}\left(Z_{2}(z)\right)\right)=R_{\mu_{2}}\left(Z_{2}(z)\right)
\end{aligned}
$$

as $z \in \mathbb{C}^{+}$. Hence $R_{\mu}^{\prime}(z) \neq 0$ as $z \in \Omega_{\mu}$. In addition note that $R_{\mu}\left(\Omega_{\mu}\right)=$ $R_{\mu_{1}}\left(\Omega_{\mu_{1}}\right) \cap R_{\mu_{2}}\left(\Omega_{\mu_{2}}\right) \supset \bar{\Gamma}_{\alpha, \beta, \Delta}^{+}$, thus proving the assertion for $\mathcal{M}_{+}^{(\alpha, \beta, \Delta)}$.

Repeating the previous arguments, using Theorem 1.7 instead of Theorem 1.4, we obtain the assertion of the proposition for $\mathcal{M}_{*}^{\alpha}$ as well which completes the proof of Proposition 6.1.

Note that every measure $\mu \in \mathbf{M}$ belongs to some set $\mathbf{M}^{\gamma}$. Introduce $\mathbf{M}^{0}:=$ $\bigcap_{\gamma \in \Gamma} \mathbf{M}^{\gamma}$.

Proposition 6.2. Let $\mu \in \mathbf{M}^{0}$.

1) If $\mu \in \mathcal{M}$, then $F_{\mu}$ has an inverse $F_{\mu}^{(-1)}$ defined on $\mathbb{C}^{+}$and

$$
F_{\mu}^{(-1)}(z)=a+z-\int_{\mathbb{R}} \frac{1+u z}{u-z} \sigma(d u), \quad z \in \mathbb{C}^{+},
$$

where $a$ is a real constant and $\sigma$ is a finite nonnegative measure. In addition $\mu \in I(\mathcal{M})$, i.e., is infinitely divisible.

2) If $\mu \in \mathcal{M}_{+}$, then $R_{\mu}$ has an inverse $R_{\mu}^{(-1)}$ defined on $\mathbb{C} \backslash \mathbb{R}_{+}$and

$$
R_{\mu}^{(-1)}(z)=z \exp \left\{a-b z-\int_{\mathbb{R}_{+}} \frac{1+u z}{u-z} \sigma(d u)\right\}, \quad z \in \mathbb{C} \backslash \mathbb{R}_{+},
$$

where $a$ and $b \geqslant 0$ are real numbers and $\sigma$ is a finite nonnegative measure. Moreover $\mu \in I\left(\mathcal{M}_{+}\right)$.

3) If $\mu \in \mathcal{M}_{*}$, then $Q_{\mu}$ has an inverse $Q_{\mu}^{(-1)}$ defined on $\mathbb{D}$ and

$$
Q_{\mu}^{(-1)}(z)=z \exp \left\{i a+\int_{\mathbb{T}} \frac{\xi+z}{\xi-z} \sigma(d \xi)\right\}, \quad z \in \mathbb{D},
$$

where $a$ is a real number and $\sigma$ is a finite nonnegative measure. In addition $\mu \in I\left(\mathcal{M}_{*}\right)$. 
Note that the assertion $\mu \in I(\mathbf{M})$ in parts 1)-3) follows from results of Bercovici and Voiculescu 3], 4. We shall give another proof based on properties of the function classes $\mathcal{N}$ and $\mathcal{C}$.

Proof. Let us prove (6.3). We conclude from the definition of $\mu \in \mathbf{M}^{0}$ that $F_{\mu}^{\prime}(z) \neq 0, z \in \mathbb{C}^{+}$, and $F_{\mu}\left(\mathbb{C}^{+}\right)=\mathbb{C}^{+}$. Hence $F_{\mu}^{(-1)}$ is defined and is analytic in some neighborhood of every point $z \in \mathbb{C}^{+}$. By the monodromy theorem, $F_{\mu}^{(-1)}(z)$ is analytic on $\mathbb{C}^{+}$. Recalling the property $\operatorname{Im} F_{\mu}(z) \geqslant \operatorname{Im} z, z \in \mathbb{C}^{+}$, we see that $\operatorname{Im} F_{\mu}^{(-1)}(z) \leqslant \operatorname{Im} z$ for $z \in \mathbb{C}^{+}$. Hence $z-F_{\mu}^{(-1)}(z)$ is a Nevanlinna function that is in $\mathcal{N}$. Moreover, as it is easy to see, $1-F_{\mu}^{(-1)}($ iy $) /($ iy $) \rightarrow$ 0 as $y \rightarrow+\infty$. Therefore we obtain representation (6.3) by the Nevanlinna integral representation (see Section 3, (3.3)). By Lemma 4.1, every function that admits an integral representation (6.3) may be written as $F_{\mu}^{(-1)}(z), z \in \mathbb{C}^{+}$, where $F_{\mu}(z), z \in \mathbb{C}^{+}$, is the reciprocal Cauchy transform of some measure $\mu \in \mathcal{M}$. Again by Lemma 4.1, the measure $\mu \in I(\mathcal{M})$.

We shall prove (6.4). It is easy to see that $R_{\mu}^{\prime}(z) \neq 0, z \in \mathbb{C}^{+}$, and $R_{\mu}\left(\mathbb{C}^{+}\right)=$ $\mathbb{C}^{+}$. Hence $R_{\mu}^{(-1)}$ is defined and is analytic in some neighborhood of every point $z \in \mathbb{C}^{+}$. Moreover $R_{\mu}^{(-1)}(z) \neq 0$ for $z \in \mathbb{C}^{+}$. By the monodromy theorem, $R_{\mu}^{(-1)}(z)$ is analytic on $\mathbb{C}^{+}$and, recalling the property $\arg R_{\mu}(z) \geqslant \arg z, z \in \mathbb{C}^{+}$ (see (2.8)), we deduce that $\arg R_{\mu}^{(-1)}(z) \leqslant \arg z$ for $z \in \mathbb{C}^{+}$. By (2.8), we note that $R_{\mu}(z)$ is analytic on $\mathbb{C} \backslash[0,+\infty), R_{\mu}^{\prime}(z)>0, z \in(-\infty, 0)$, and $R_{\mu}(\bar{z})=\overline{R_{\mu}(z)}$, $z \in \mathbb{C}^{+}$. Therefore we conclude that $R_{\mu}^{(-1)}(z)$ is analytic on $\mathbb{C} \backslash[0,+\infty)$ and $\arg R_{\mu}^{(-1)}(z) \geqslant \arg z$ for $z \in \mathbb{C}^{-}$. Since $R_{\mu}^{(-1)}(z) \neq 0$ for $z \in \mathbb{C} \backslash[0,+\infty)$, we see that $\log R_{\mu}^{(-1)}(z)$ is analytic on $\mathbb{C} \backslash[0,+\infty)$ and $\operatorname{Im} \log \left(R_{\mu}^{(-1)}(z) / z\right) \leqslant 0$ for $z \in \mathbb{C}^{+}$ and $\operatorname{Im} \log \left(R_{\mu}^{(-1)}(z) / z\right) \geqslant 0$ for $z \in \mathbb{C}^{-}$. (Recall that we take the principal branch of the logarithm). Hence, by the integral representation of Nevanlinna functions (3.3), we obtain

$$
\log \left(R_{\mu}^{(-1)}(z) / z\right)=a-b z-\int_{\mathbb{R}} \frac{1+u z}{u-z} \sigma(d u), \quad z \in \mathbb{C}^{+},
$$

where $a \in \mathbb{R}, b \geqslant 0$, and $\sigma$ is a finite nonnegative measure. Since $R_{\mu}^{(-1)}(z) / z$ is analytic and real on the negative real axis, it follows via the inversion formula (3.7) that $\sigma\left(\mathbb{R} \backslash \mathbb{R}^{+}\right)=0$. Thus (6.4) is proved. By Lemma 5.3, every function that admits an integral representation of type (6.4) may be written as $R_{\mu}^{(-1)}(z), z \in \mathbb{C}^{+}$, where $R_{\mu}(z), z \in \mathbb{C}^{+}$, belongs to the class $\mathcal{K}$. Hence it follows that the measure $\mu \in I\left(\mathcal{M}_{+}\right)$.

Let us prove (6.5). Note that $Q_{\mu}^{\prime}(z) \neq 0, z \in \mathbb{D}, Q_{\mu}(0)=0$, and $Q_{\mu}(\mathbb{D})=$ $\mathbb{D}$. Hence $Q_{\mu}^{(-1)}$ is defined and is analytic in some neighborhood of every point $z \in \mathbb{D}$. By the monodromy theorem, $Q_{\mu}^{(-1)}(z)$ is analytic on $\mathbb{D}, Q_{\mu}^{(-1)}(0)=$ 0 , and, using $\left|Q_{\mu}(z)\right| \leqslant 1, z \in \mathbb{D}$, we see that $\left|Q_{\mu}^{(-1)}(z) / z\right| \geqslant 1$ as $z \in \mathbb{D}$. 
Hence $\log \left(Q_{\mu}^{(-1)}(z) / z\right)$ is analytic on $\mathbb{D}$ and $\operatorname{Re} \log \left(Q_{\mu}^{(-1)}(z) / z\right) \geqslant 0, z \in \mathbb{D}$. Thus, the function $\log \left(Q_{\mu}^{(-1)}(z) / z\right)$ belongs to the Carathéodory class $\mathcal{C}$ and we obtain the representation (6.5). By Lemma 5.6, every function that admits an integral representation (6.5) may be written as $Q_{\mu}^{(-1)}(z), z \in \mathbb{D}$, where $Q_{\mu}(z), z \in \mathbb{D}$ belongs to the class $\mathcal{S}_{*}$. Hence we obtain that the measure $\mu \in I\left(\mathcal{M}_{*}\right)$.

The proposition is proved.

Proposition 6.3. For any fixed $\gamma$ the semigroup $\left(\mathbf{M}^{\gamma}, \circ\right)$ is hereditary in $(\mathbf{M}, \circ)$.

Proof. Consider first the semigroup $(\mathcal{M}, \boxplus)$. Let $\mu=\mu_{1} \boxplus \mu_{2}$, where $\mu \in$ $\mathcal{M}^{(\alpha, \beta)}$. Then, by Theorem 2.1, there exist functions $Z_{1}(z)$ and $Z_{2}(z)$ of class $\mathcal{F}$ such that $F_{\mu}(z)=F_{\mu_{1}}\left(Z_{1}(z)\right)=F_{\mu_{2}}\left(Z_{2}(z)\right)$ as $z \in \mathbb{C}^{+}$. Since the relation $F_{\mu}^{\prime}(z)=F_{\mu_{1}}^{\prime}\left(Z_{1}(z)\right) Z_{1}^{\prime}(z) \neq 0$ holds for $z \in \Omega_{\mu}$, we conclude $F_{\mu_{1}}^{\prime}(\zeta) \neq 0$ for $\zeta \in \Omega_{\mu_{1}}:=Z_{1}\left(\Omega_{\mu}\right)$. In addition $F_{\mu_{1}}\left(\Omega_{\mu_{1}}\right) \supset \bar{\Gamma}_{\alpha, \beta}$. Hence $\mu_{1} \in \mathcal{M}^{(\alpha, \beta)}$. In the same way we may show that $\mu_{2} \in \mathcal{M}^{(\alpha, \beta)}$ as well thus proving the proposition for $(\mathcal{M}, \boxplus)$.

Consider the semigroup $\left(\mathcal{M}_{+}, \nabla\right)$. Let $\mu=\mu_{1} \otimes \mu_{2}$, where $\mu \in \mathcal{M}_{+}^{(\alpha, \beta, \Delta)}$. By Theorem 2.4, there exist functions $Z_{1}(z)$ and $Z_{2}(z)$ of class $\mathcal{K}$ such that $R_{\mu}(z)=$ $R_{\mu_{1}}\left(Z_{1}(z)\right)=R_{\mu_{2}}\left(Z_{2}(z)\right)$ as $z \in \mathbb{C}^{+}$. Since $R_{\mu}^{\prime}(z)=R_{\mu_{1}}^{\prime}\left(Z_{1}(z)\right) Z_{1}^{\prime}(z) \neq 0$ for $z \in$ $\Omega_{\mu}$, we have $R_{\mu_{1}}^{\prime}(\zeta) \neq 0$ for $\zeta \in \Omega_{\mu_{1}}:=Z_{1}\left(\Omega_{\mu}\right)$. In addition $R_{\mu_{1}}\left(\Omega_{\mu_{1}}\right) \supset \bar{\Gamma}_{\alpha, \beta, \Delta}^{+}$. Hence $\mu_{1} \in \mathcal{M}_{+}^{(\alpha, \beta, \Delta)}$. Similarly we get $\mu_{2} \in \mathcal{M}^{(\alpha, \beta, \Delta)}$ as well which concludes the proof for $\left(\mathcal{M}_{+}, \boxplus\right)$.

We shall finally consider the semigroup $\left(\mathcal{M}_{*}, \nabla\right)$. Let $\mu=\mu_{1} \otimes \mu_{2}$, where $\mu \in$ $\mathcal{M}_{*}^{\alpha}$. By Theorem 2.7, there exist functions $Z_{1}(z)$ and $Z_{2}(z)$ of class $\mathcal{S}_{*}$ such that $Q_{\mu}(z)=Q_{\mu_{1}}\left(Z_{1}(z)\right)=Q_{\mu_{2}}\left(Z_{2}(z)\right)$ as $z \in \mathbb{D}$. Since $Q_{\mu}^{\prime}(z)=Q_{\mu_{1}}^{\prime}\left(Z_{1}(z)\right) Z_{1}^{\prime}(z) \neq 0$ for $z \in \Omega_{\mu}$, we have $Q_{\mu_{1}}^{\prime}(\zeta) \neq 0$ for $\zeta \in \Omega_{\mu_{1}}:=Z_{1}\left(\Omega_{\mu}\right)$. In addition $Q_{\mu_{1}}\left(\Omega_{\mu_{1}}\right) \supset$ $\overline{\mathbb{D}}_{\alpha}$. Hence $\mu_{1} \in \mathcal{M}_{*}^{\alpha}$. Similar as above we deduce $\mu_{2} \in \mathcal{M}_{*}^{\alpha}$ thus proving Proposition 6.3.

Define an equivalence relation $E$ on $\mathbf{M}$ via $\mu E \nu$ if $\mu=\nu \circ \delta_{a}$, where $\delta_{a}$ is Dirac measure with the support at the point $a$. Here $a$ denotes a real number for $(\mathcal{M}, \boxplus)$, is a positive number for $\left(\mathcal{M}_{+}, \otimes\right)$ and $a \in \mathbb{T}$ for $\left(\mathcal{M}_{*}, \otimes\right)$. It is clear that $E$ is an equivalence relation on the subsemigroup $\mathbf{M}^{\gamma}$ as well. We shall denote the equivalence class of $\mu \in \mathbf{M}$ as $E \mu$, and the element of $\mathbf{M} / E$ by $\mu^{*}, \nu^{*}$ etc. Note that $\mathbf{M} / E$ (and similarly $\mathbf{M}^{\gamma} / E$ ) is a semigroup under $\circ$ multiplication of representatives.

Define a homomorphism $D^{\gamma}$ of $\mathbf{M}^{\gamma} / E$ to non-negative real numbers with addition in the following way.

For the semigroup $(\mathcal{M}, \boxplus)$ :

$D^{\gamma}\left(\mu^{*}\right):=-\operatorname{Im} \varphi_{\nu}(i(\beta+1))$, where $\nu=E \mu$ and $\nu \in \mathcal{M}^{(\alpha, \beta)}$.

For the semigroup $\left(\mathcal{M}_{+}, \otimes\right)$ :

$D^{\gamma}\left(\mu^{*}\right):=-\operatorname{Im} \log \Sigma_{\nu}\left((\beta+\Delta) e^{i(\pi+\alpha) / 2} / 2\right)$, where $\nu=E \mu$ and $\nu \in \mathcal{M}_{+}^{(\alpha, \beta, \Delta)}$. 
For the semigroup $\left(\mathcal{M}_{*}, \nabla\right)$ :

$D^{\gamma}\left(\mu^{*}\right):=\operatorname{Re} \log \Sigma_{\nu}(\alpha / 2)$, where $\nu=E \mu$ and $\nu \in \mathcal{M}_{*}^{\alpha}$.

Note that $D^{\gamma}$ is finite-valued and does not depend on the particular $\nu$.

For $\mu^{*}, \nu^{*} \in \mathbf{M} / E$ define a distance $L^{*}\left(\mu^{*}, \nu^{*}\right)$ via

$$
L^{*}\left(\mu^{*}, \nu^{*}\right)=\inf \left\{L(\mu, \nu): E \mu=\mu^{*}, E \nu=\nu^{*}\right\},
$$

where $L(\mu, \nu)$ denotes the Lévy metric. It is easy to check that $L^{*}\left(\mu^{*}, \nu^{*}\right)$ is a metric, and that $\left\{\mu_{n}^{*}\right\}$ converges to $\mu^{*} \in \mathbf{M} / E$ with respect to $L^{*}$ if and only if there is $\left\{\nu_{n}\right\}$ with $\nu_{n} \in \mathbf{M}$ and $\mu_{n}^{*}=E \nu_{n}$ for each $n$, and $\nu \in \mathbf{M}$ with $\mu^{*}=E \nu$, such that $\left\{\nu_{n}\right\}$ converges to $\nu$ with respect to $L$.

Recall that $M$ denoted the set of sequences of elements of $\mathbf{M}$ with weak limits in $\mathbf{M}$, and $l$ denotes the operation of taking the weak limit. We denote by $M / E$ the set of sequences of elements of $\mathbf{M} / E$ that admit limits with respect to $L^{*}$ and by $l$ again the operation of taking the limit.

Proposition 6.4. The operation o is continuous with respect to Lévy's metric.

This result is due to Bercovici and Voiculescu [3, 4]. For convenience of the reader we include a proof.

Proof of Proposition 6.4 Let $\left\{\mu_{n}\right\}$ and $\left\{\nu_{n}\right\}$ be in $M$. Let us prove that $\left\{\mu_{n} \boxplus\right.$ $\left.\nu_{n}\right\} \in M$ and $l\left\{\mu_{n} \boxplus \nu_{n}\right\}=l\left\{\mu_{n}\right\} \boxplus l\left\{\nu_{n}\right\}$.

At first consider the semigroup $(\mathcal{M}, \boxplus)$. By Proposition 3.11, the sequences $\left\{\varphi_{\mu_{n}}\right\}$ and $\left\{\varphi_{\nu_{n}}\right\}$ converge uniformly on compact subsets of some $\Gamma_{\alpha, \beta}$ to the functions $\varphi_{\mu}$ and $\varphi_{\nu}$, and $\varphi_{\mu_{n}}(i y)=o(y), \varphi_{\nu_{n}}(i y)=o(y)$ uniformly in $n$ as $y \rightarrow+\infty$. Since $\varphi_{\mu_{n} \boxplus \nu_{n}}(z)=\varphi_{\mu_{n}}(z)+\varphi_{\nu_{n}}(z)$ and $\varphi_{\mu \boxplus \nu}(z)=\varphi_{\mu}(z)+\varphi_{\nu}(z)$ for $z \in \Gamma_{\alpha, \beta}$, note that $\left\{\varphi_{\mu_{n} \boxplus \nu_{n}}\right\}$ converges uniformly on compact subsets of some $\Gamma_{\alpha, \beta}$ to the function $\varphi_{\mu \boxplus \nu}$. Furthemore, $\varphi_{\mu_{n} \boxplus \nu_{n}}(i y)=o(y)$ uniformly in $n$ as $y \rightarrow+\infty$. The assertion for $(\mathcal{M}, \boxplus)$ now follows immediately from Proposition 3.11.

Consider the semigroup $\left(\mathcal{M}_{+}, \nabla\right)$. Assume that the sequences $\left\{\mu_{n}\right\}$ and $\left\{\nu_{n}\right\}$ converge in the weak topology to measures $\mu \neq \delta_{0}$ and $\nu \neq \delta_{0}$, respectively. Since $\Sigma_{\mu_{n} \unrhd \nu_{n}}=\Sigma_{\mu_{n}} \Sigma_{\nu_{n}}$ on the set, where these functions are defined, we easily conclude from Proposition 3.12 that the sequence $\left\{\mu_{n} \otimes \nu_{n}\right\}$ converges as well to a p-measure which is not the Dirac measure $\delta_{0}$. By Proposition 3.12 there exist numbers $\alpha \in(0, \pi)$ and $0<\beta<\Delta$ such that the sequences $\left\{\Sigma_{\mu_{n}}\right\}$ and $\left\{\Sigma_{\nu_{n}}\right\}$ converge uniformly on $\Gamma_{\alpha, \beta, \Delta}^{+}$to the functions $\Sigma_{\mu}$ and $\Sigma_{\nu}$, respectively. Hence, $\left\{\Sigma_{\mu_{n}} \Sigma_{\nu_{n}}\right\}$ converges uniformly on $\Gamma_{\alpha, \beta, \Delta}^{+}$to the function $\Sigma_{\mu} \Sigma_{\nu}$ and, by Proposition [3.12, we conclude that $\left\{\mu_{n} \otimes \nu_{n}\right\}$ converges weakly to $\mu \otimes \nu \neq \delta_{0}$, which is the desired result.

It remains to consider the semigroup $\left(\mathcal{M}_{*}, \otimes\right)$. By Proposition 3.13, the sequences $\left\{\Sigma_{\mu_{n}}\right\}$ and $\left\{\Sigma_{\nu_{n}}\right\}$ converge uniformly in some neighborhood of zero, say $\mathbb{D}_{\alpha}, \alpha \in(0,1]$, to the functions $\Sigma_{\mu}$ and $\Sigma_{\nu}$, respectively. Since $\Sigma_{\mu_{n} \bowtie \nu_{n}}=\Sigma_{\mu_{n}} \Sigma_{\nu_{n}}$ in $\mathbb{D}_{\alpha}$, we conclude that $\left\{\Sigma_{\mu_{n} \bowtie \nu_{n}}\right\}$ converge uniformly to $\Sigma_{\mu} \Sigma_{\nu}$. Applying Proposition 3.13 , we arrive at the assertion of the proposition for $\left(\mathcal{M}_{*}, \nabla\right)$. 
In the sequel we denote $a(\mu):=-\operatorname{Re} \varphi_{\mu}(i(\beta+1))$ for $\mu \in \mathcal{M}, a(\mu):=$ $1 /\left|\Sigma_{\mu}\left((\beta+\Delta) e^{i(\pi+\alpha) / 2} / 2\right)\right|$ for $\mu \in \mathcal{M}_{+}$, and $a(\mu):=\exp \left\{-i \arg \Sigma_{\mu}(\alpha / 2)\right\}$ for $\mu \in \mathcal{M}_{*}$.

Proposition 6.5. If $\left\{\mu_{n}^{*}\right\}$ is any element of $M / E$, and if, for each $n \in \mathbb{N}, \nu_{n}^{*}$ is a factor of $\mu_{n}^{*}$, then there is a subsequence $\left\{n^{\prime}\right\}$ of $\{n\}$ such that $\left\{\nu_{n^{\prime}}^{*}\right\} \in M / E$ and $l\left\{\nu_{n^{\prime}}^{*}\right\}$ is a factor of $l\left\{\mu_{n}^{*}\right\}$.

Proof. Let $\left\{\mu_{n}^{*}\right\}$ converges to $\mu^{*} \in \mathbf{M} / E$ in the metric $L^{*}$, and let $\nu_{n}^{*}$ be a factor of $\mu_{n}^{*}$ for each $n$. Then for each $n$ there exist probability measures $\mu_{n}$ and $\nu_{n}$, such that $\mu_{n}^{*}=E \mu_{n}$ and $\nu_{n}^{*}=E \nu_{n}, \nu_{n}$ is a M-factor of $\mu_{n}$, and $\left\{\mu_{n}\right\}$ converges to $\mu$ in the metric $L$, where $\mu \in \mathbf{M}$ and $\mu^{*}=E \mu$.

At first let us prove the assertion of the proposition for $(\mathcal{M}, \boxplus)$. By Proposition 3.11, the sequence $\left\{\varphi_{\mu_{n}}\right\}$ converges uniformly on compact subsets of some set $\Gamma_{\alpha, \beta}$ to the function $\varphi_{\mu}$, and $\varphi_{\mu_{n}}(i y)=o(y)$ uniformly in $n$ as $y \rightarrow+\infty$. Since, for $\mu \in \mathcal{M}^{(\alpha, \beta)},-\operatorname{Im} \varphi_{\mu}(z) \geqslant 0, z \in \Gamma_{\alpha, \beta}$, we have $-\operatorname{Im} \varphi_{\nu_{n}}(z) \leqslant$ $-\operatorname{Im} \varphi_{\mu_{n}}(z), z \in \Gamma_{\alpha, \beta}$. By Theorem 3.8. there exists a subsequence $\left\{n^{\prime}\right\}$ of $\{n\}$ such that $-\operatorname{Im} \varphi_{\nu_{n^{\prime}}}(z)$ converges uniformly on compact subsets of $\Gamma_{\alpha, \beta}$ to a harmonic function $U(z) \geqslant 0$. Moreover $-\operatorname{Im} \varphi_{\nu_{n^{\prime}}}(i y)=o(y)$ uniformly in $n$ as $y \rightarrow+\infty$. Consider the sequence of analytic functions $\left\{\varphi_{\nu_{n^{\prime}}}(z)+a\left(\nu_{n^{\prime}}\right)\right\}=$ $\left\{\varphi_{\nu_{n^{\prime}} \boxplus \delta_{a\left(\nu_{n^{\prime}}\right)}}(z)\right\}$ in $\Gamma_{\alpha, \beta}$. This sequence converges at the point $i(\beta+1)$ and hence, by Theorem 3.9] $\left\{\varphi_{\nu_{n^{\prime}}}(z)+a\left(\nu_{n^{\prime}}\right)\right\}$ converges uniformly on every compact subset of $\Gamma_{\alpha, \beta}$. Hence the assumptions of Proposition 3.11 hold for the sequence $\left\{\nu_{n^{\prime}} \boxplus \delta_{a\left(\nu_{n^{\prime}}\right)}\right\}$. By this proposition, this sequence of p-measures converges in the weak topology to a p-measure $\nu$. Iterating this argument, we see that there is a subsequence $\left\{n^{\prime \prime}\right\} \subset\left\{n^{\prime}\right\}$ such that $\left\{\rho_{n^{\prime \prime}}\right\} \rightarrow \rho$, say, where $\rho_{n^{\prime \prime}}$ is some cofactor of $\nu_{n^{\prime \prime}} \boxplus \delta_{a\left(\nu_{n^{\prime \prime}}\right)}$ in $\mu_{n^{\prime \prime}}$. By continuity of the additive free convolution (Proposition 6.4), we have $\nu \boxplus \rho=\mu$, so $\nu$ is a factor of $\mu$. Thus Proposition 6.5 is proved for $(\mathcal{M}, \boxplus)$.

Now we shall prove the assertion for $\left(\mathcal{M}_{+}, \bigotimes\right)$. By Proposition 3.12, the sequence $\left\{\Sigma_{\mu_{n}}\right\}$ converges uniformly on compact subsets of some $\Gamma_{\alpha, \beta, \Delta}^{+}$to the function $\Sigma_{\mu}$. Recall that for every $\mu \in \mathcal{M}_{+}^{(\alpha, \beta, \Delta)}$ the function $\log \Sigma_{\mu}(z)$ is analytic in $\Gamma_{\alpha, \beta, \Delta}^{+}$and, in addition, $-\operatorname{Im} \log \Sigma_{\mu}(z) \geqslant 0$ for $z \in \Gamma_{\alpha, \beta, \Delta}^{+} \backslash \mathbb{C}^{-}$and $\operatorname{Im} \log \Sigma_{\mu}(\bar{z})=$ $-\operatorname{Im} \log \Sigma_{\mu}(z)$ for $z \in \Gamma_{\alpha, \beta, \Delta}^{+}$. Since $-\operatorname{Im} \log \Sigma_{\nu_{n}}(z) \leqslant-\operatorname{Im} \log \Sigma_{\mu_{n}}(z)$ for $z \in$ $\Gamma_{\alpha, \beta, \Delta}^{+} \backslash \mathbb{C}^{-}$, we may apply Theorem 3.8 to $\left\{-\operatorname{Im} \log \Sigma_{\nu_{n}}(z)\right\}$ and obtain that there exists a subsequence $\left\{n^{\prime}\right\}$ of $\{n\}$ such that $\operatorname{Im} \log \Sigma_{\nu_{n^{\prime}}}(z)$ converges uniformly on the compact subsets of $\Gamma_{\alpha, \beta, \Delta}^{+}$to a harmonic function $U(z)$. Consider the sequence of analytic functions $\left\{\log \left(\Sigma_{\nu_{n^{\prime}}}(z) a\left(\nu_{n^{\prime}}\right)\right)\right\}=\left\{\log \Sigma_{\nu_{n^{\prime}}} \otimes \delta_{a\left(\nu_{n^{\prime}}\right)}(z)\right\}$ in $\Gamma_{\alpha, \beta, \Delta}^{+}$. This sequence converges at the point $(\beta+\Delta) e^{i(\pi+\alpha) / 2} / 2$ and then, by Theorem [3.9. $\left\{\Sigma_{\nu_{n^{\prime}} \otimes \delta_{a\left(\nu_{n^{\prime}}\right)}}(z)\right\}$ converges uniformly on every compact subset of $\Gamma_{\alpha, \beta, \Delta}^{+}$. Hence the assumptions of Proposition 3.12 hold for the sequence $\left\{\nu_{n^{\prime}} \otimes \delta_{a\left(\nu_{n^{\prime}}\right)}\right\}$ and the sequence of p-measures converges in the weak topology to a p-measure $\nu$. 
Iterating this argument, we see that there is a subsequence $\left\{n^{\prime \prime}\right\} \subset\left\{n^{\prime}\right\}$ such that $\left\{\rho_{n^{\prime \prime}}\right\} \rightarrow \rho$, say, where $\rho_{n^{\prime \prime}}$ is some cofactor of $\nu_{n^{\prime \prime}} \otimes \delta_{a\left(\nu_{n^{\prime \prime}}\right)}$ in $\mu_{n^{\prime \prime}}$. By continuity of the multiplicative free convolution (Proposition 6.4), we have $\nu \boxplus \rho=\mu$, so $\nu$ is a factor of $\mu$. Thus Proposition 6.5 is proved for $\left(\mathcal{M}_{+}, \nabla\right)$.

It remains to consider the case of the semigroup $\left(\mathcal{M}_{*}, \nabla\right)$. By Proposition 3.13, the sequence $\left\{\Sigma_{\mu_{n}}\right\}$ converges uniformly on compact subsets of some $\mathbb{D}_{\alpha}$ to the function $\Sigma_{\mu}$. Recall that for every $\mu \in \mathcal{M}_{*}^{\alpha}$ the function $\log \Sigma_{\mu}(z)$ is analytic in $\mathbb{D}_{\alpha}$ and Re $\log \Sigma_{\mu}(z)=\log \left|\Sigma_{\mu}(z)\right| \geqslant 0$ for $z \in \mathbb{D}_{\alpha}$. Since $\log \left|\Sigma_{\nu_{n}}(z)\right| \leqslant$ $\log \left|\Sigma_{\mu_{n}}(z)\right|$ for $z \in \mathbb{D}_{\alpha}$, we may apply Theorem 3.8 to $\left\{\log \left|\Sigma_{\nu_{n}}(z)\right|\right\}$ and obtain that there exists a subsequence $\left\{n^{\prime}\right\}$ of $\{n\}$ such that $\left\{\log \left|\Sigma_{\nu_{n^{\prime}}}(z)\right|\right\}$ converges uniformly on compact subsets of $\mathbb{D}_{\alpha}$ to a harmonic function $U(z) \geqslant 0$. Consider the sequence of analytic functions in $\mathbb{D}_{\alpha}\left\{\log \left(\Sigma_{\nu_{n^{\prime}}}(z) a_{n^{\prime}}\right)\right\}=\left\{\log \Sigma_{\nu_{n^{\prime}}} \otimes \delta_{a\left(\nu_{n^{\prime}}\right)}(z)\right\}$. This sequence converges at the point $\alpha / 2$ and thus, by Theorem 3.9, the sequence $\left\{\Sigma_{\nu_{n^{\prime}}} \otimes \delta_{a\left(\nu_{n^{\prime}}\right)}(z)\right\}$ converges uniformly on every compact subset of $\mathbb{D}_{\alpha}$. Hence the assumptions of Proposition 3.13 hold for the sequence $\left\{\nu_{n^{\prime}} \otimes \delta_{a\left(\nu_{n^{\prime}}\right)}\right\}$. By this proposition, this sequence of p-measures converges in the weak topology to a p-measure $\nu$. Iterating the argument, we see that there is a subsequence $\left\{n^{\prime \prime}\right\} \subset\left\{n^{\prime}\right\}$ such that $\left\{\rho_{n^{\prime \prime}}\right\} \rightarrow \rho$, say, where $\rho_{n^{\prime \prime}}$ is some cofactor of $\nu_{n^{\prime \prime}} \otimes \delta_{a\left(\nu_{n^{\prime \prime}}\right)}$ in $\mu_{n^{\prime \prime}}$. By continuity of the multiplicative free convolution (Proposition 6.3), we have $\nu \boxplus \rho=\mu$, so $\nu$ is a factor of $\mu$, thus proving Proposition 6.5 for $\left(\mathcal{M}_{*}, \bigotimes\right)$.

Proposition 6.5 is completely proved.

Proposition 6.6. Let $\left\{\nu_{n}\right\}$ be a sequence of p-measures $\nu_{n} \in \mathbf{M}^{\gamma}$ and let $D^{\gamma}\left(\nu_{n}^{*}\right) \rightarrow$ 0 as $n \rightarrow \infty$. For every fixed $\gamma^{\prime}$ there exists $n\left(\gamma^{\prime}\right)$ such that $\nu_{n} \circ \delta_{a\left(\nu_{n}\right)} \in \mathbf{M}^{\gamma^{\prime}}$ for $n \geqslant n\left(\gamma^{\prime}\right)$.

Proof. Let us prove the assertion for the semigroup $(\mathcal{M}, \boxplus)$. Let $\left\{\nu_{n}\right\}$ denote a sequence of p-measures $\nu_{n} \in \mathcal{M}^{(\alpha, \beta)}$ and let $\operatorname{Im} \varphi_{\nu_{n}}\left(z_{0}\right) \rightarrow 0$ as $n \rightarrow \infty$, where $z_{0}:=i(\beta+1)$. We shall prove that for every fixed $\alpha^{\prime}>\alpha$ and $0<\beta^{\prime}<\beta$, there exists $n\left(\alpha^{\prime}, \beta^{\prime}\right)$ such that $\nu_{n} \boxplus \delta_{a\left(\nu_{n}\right)} \in \mathcal{M}^{\left(\alpha^{\prime}, \beta^{\prime}\right)}$ for $n \geqslant n\left(\alpha^{\prime}, \beta^{\prime}\right)$.

The functions $\operatorname{Im} \varphi_{\nu_{n}}(z)$ are harmonic non positive-valued functions in $\Gamma_{\alpha, \beta}$. Therefore, by the theorem of the mean for harmonic functions,

$$
\left|\operatorname{Im} \varphi_{\nu_{n}}\left(z_{0}\right)\right|=\frac{1}{2 \pi} \int_{0}^{2 \pi}\left|\operatorname{Im} \varphi_{\nu_{n}}\left(z_{0}+\varepsilon e^{i \theta}\right)\right| d \theta
$$

for sufficiently small $\varepsilon>0$. Since $\operatorname{Re} \varphi_{\nu_{n} \boxplus \delta_{a\left(\nu_{n}\right)}}\left(z_{0}\right)=0$, we have, by Schwarz's formula (see Markushevich (1965), v. 2, p. 151),

$$
\varphi_{\nu_{n} \boxplus \delta_{a\left(\nu_{n}\right)}}(z)=\frac{i}{2 \pi} \int_{0}^{2 \pi} \operatorname{Im} \varphi_{\nu_{n}}\left(z_{0}+\varepsilon e^{i \theta} / 2\right) \frac{\varepsilon e^{i \theta} / 2+\left(z-z_{0}\right)}{\varepsilon e^{i \theta} / 2-\left(z-z_{0}\right)} d \theta
$$


for $z \in \mathbb{C}$ such that $\left|z-z_{0}\right|<\varepsilon / 2$. Using (6.6), we obtain from this formula, for $\left|z-z_{0}\right| \leqslant \varepsilon / 4$,

$$
\begin{aligned}
\left|\varphi_{\nu_{n} \boxplus \delta_{a\left(\nu_{n}\right)}}(z)\right| & \leqslant \frac{1}{2 \pi} \int_{0}^{2 \pi}\left|\operatorname{Im} \varphi_{\nu_{n}}\left(z_{0}+\varepsilon e^{i \theta} / 2\right)\right| \frac{\varepsilon / 2+\left|z-z_{0}\right|}{\varepsilon / 2-\left|z-z_{0}\right|} d \theta \\
& \leqslant \frac{3}{2 \pi} \int_{0}^{2 \pi}\left|\operatorname{Im} \varphi_{\nu_{n}}\left(z_{0}+\varepsilon e^{i \theta} / 2\right)\right| d \theta=3\left|\operatorname{Im} \varphi_{\nu_{n}}\left(z_{0}\right)\right| .
\end{aligned}
$$

Since $\left|\operatorname{Im} \varphi_{\nu_{n}}\left(z_{0}\right)\right| \rightarrow 0$ as $n \rightarrow \infty$, we conclude from (6.7) that $\varphi_{\nu_{n} \boxplus \delta_{a\left(\nu_{n}\right)}}(z) \rightarrow$ 0 uniformly for $\left|z-z_{0}\right| \leqslant \varepsilon / 4$ as $n \rightarrow \infty$. Recall that $F_{\nu_{n} \boxplus \delta_{a\left(\nu_{n}\right)}^{(-1)}}^{(z)=z+}$ $\varphi_{\nu_{n} \boxplus \delta_{a\left(\nu_{n}\right)}}(z)$. Therefore we easily conclude that $F_{\nu_{n} \boxplus \delta_{a\left(\nu_{n}\right)}}=z+o(1)$ and hence $G_{\nu_{n} \boxplus \delta_{a\left(\nu_{n}\right)}}(z)=1 / z+o(1)$ for $\left|z-z_{0}\right| \leqslant \varepsilon / 8$. From the last relation we see that (6.8)

$\operatorname{Im}\left(G_{\nu_{n} \boxplus \delta_{a\left(\nu_{n}\right)}}\left(z_{0}\right)-\frac{1}{z_{0}}\right)=\frac{1}{\operatorname{Im} z_{0}} \int_{\mathbb{R}} \frac{u^{2}}{u^{2}+\left(\operatorname{Im} z_{0}\right)^{2}} \nu_{n} \boxplus \delta_{a\left(\nu_{n}\right)}(d u) \rightarrow 0$, as $n \rightarrow \infty$.

From (6.8) it follows that $\nu_{n} \boxplus \delta_{a\left(\nu_{n}\right)}$ converges weakly to $\delta_{0}$ as $n \rightarrow \infty$. The last relation easily implies the assertion of Proposition 6.6 for $(\mathcal{M}, \boxplus)$.

We shall consider the assertion for the semigroup $\left(\mathcal{M}_{+}, \nabla\right)$. Let $\left\{\nu_{n}\right\}$ denote a sequence of p-measures $\nu_{n} \in \mathcal{M}_{+}^{(\alpha, \beta, \Delta)}$ and assume that $\operatorname{Im} \log \Sigma_{\nu_{n}}\left(z_{0}\right) \rightarrow 0$ as $n \rightarrow \infty$, where $z_{0}:=(\beta+\Delta) e^{i(\pi+\alpha) / 2} / 2$. We shall prove that for every fixed $0<\alpha^{\prime}<\alpha, 0<\beta^{\prime}<\beta$, and $\Delta^{\prime}>\Delta$, there exists $n\left(\alpha^{\prime}, \beta^{\prime}, \Delta^{\prime}\right)$ such that $\nu_{n} \boxplus \delta_{a\left(\nu_{n}\right)} \in \mathcal{M}^{\left(\alpha^{\prime}, \beta^{\prime}, \Delta^{\prime}\right)}$ for $n \geqslant n\left(\alpha^{\prime}, \beta^{\prime}, \Delta^{\prime}\right)$.

The functions $\operatorname{Im} \log \Sigma_{\nu_{n}}(z)$ are harmonic non positive-valued functions in the domain $\Gamma_{\alpha, \beta, \Delta}^{+} \cap \mathbb{C}^{+}$such that $\log \left|\Sigma_{\nu_{n} \bowtie \delta_{a\left(\nu_{n}\right)}}\left(z_{0}\right)\right|=0$. Repeating the previous arguments for the analytic functions $\log \Sigma_{\nu_{n}} \bowtie \delta_{a\left(\nu_{n}\right)}(z)$ on $\Gamma_{\alpha, \beta, \Delta}^{+}$, we see that these functions $\log \Sigma_{\nu_{n} \otimes \delta_{a\left(\nu_{n}\right)}}(z) \rightarrow 0$ uniformly for $\left|z-z_{0}\right| \leqslant \varepsilon / 4$ as $n \rightarrow \infty$, where as above $\varepsilon>0$ is chosen sufficiently small. Hence $\Sigma_{\nu_{n} \otimes \delta_{a\left(\nu_{n}\right)}}(z)$ converges to 1 uniformly for $\left|z-z_{0}\right| \leqslant \varepsilon / 4$ as $n \rightarrow \infty$. Recalling the definition of $\Sigma_{\nu_{n} \bowtie \delta_{a\left(\nu_{n}\right)}}(z)$, we conclude from this relation that

$$
R_{\nu_{n} \unrhd \delta_{a\left(\nu_{n}\right)}}(z) \rightarrow z
$$

uniformly for $\left|z-z_{0}\right| \leqslant \varepsilon / 8$ as $n \rightarrow \infty$. Taking into account representation (2.8) for the functions $R_{\nu_{n} \bowtie \delta_{a\left(\nu_{n}\right)}}$, we have

$$
\frac{1}{z} R_{\nu_{n} \otimes \delta_{a\left(\nu_{n}\right)}}(z)=a_{n}+\int_{(0, \infty)} \frac{\tau_{n}(d t)}{t-z}, \quad z \in \mathbb{C}^{+},
$$


where $a_{n} \geqslant 0$ and the nonnegative measures $\tau_{n}$ satisfy (2.9). We easily see from (6.9) and (6.10) that

$$
a_{n}+\int_{(0, \infty)} \frac{\tau_{n}(d t)}{t+1} \leqslant c\left(z_{0}\right)<\infty, \quad n=1,2, \ldots,
$$

where $c\left(z_{0}\right)$ is a positive constant depending on $z_{0}$. This estimate implies the inequality $\left|R_{\nu_{n} \otimes \delta_{a\left(\nu_{n}\right)}}(z) / z\right| \leqslant c\left(\alpha^{\prime}, \beta^{\prime}, \Delta^{\prime}\right), n=1,2, \ldots$, in every domain $\Gamma_{\alpha^{\prime}, \beta^{\prime}, \Delta^{\prime}}^{+}$ with $\alpha^{\prime}<\alpha, \beta^{\prime}<\beta$, and $\Delta^{\prime}>\Delta$. Here $c\left(\alpha^{\prime}, \beta^{\prime}, \Delta^{\prime}\right)$ denotes a positive constant depending on $\alpha^{\prime}, \beta^{\prime}$, and $\Delta^{\prime}$. Hence, by (6.10), we conclude with the help of Vitali's theorem (see Theorem 3.10 of Section 3) that relation (6.9) holds for $z \in \Gamma_{\alpha^{\prime}, \beta^{\prime}, \Delta^{\prime}}^{+}$. From this relation we immediately obtain the assertion for $\left(\mathcal{M}_{+}, \bigotimes\right)$.

Let us prove the assertion for $\left(\mathcal{M}_{*}, \otimes\right)$. Let $\left\{\nu_{n}\right\}$ be a sequence of p-measures $\nu_{n} \in \mathcal{M}_{*}^{\alpha}$ such that let $\log \left|\Sigma_{\nu_{n}}\left(z_{0}\right)\right| \rightarrow 0$ as $n \rightarrow \infty$. Here $z_{0}:=\alpha / 2$. We shall prove that for every fixed $\alpha^{\prime}>\alpha$ there exists $n\left(\alpha^{\prime}\right)$ such that $\nu_{n} \boxplus \delta_{a\left(\nu_{n}\right)} \in \mathcal{M}^{\alpha^{\prime}}$ for $n \geqslant n\left(\alpha^{\prime}\right)$.

The functions $\log \left|\Sigma_{\nu_{n}}(z)\right|$ are harmonic non negative-valued functions on $\mathbb{D}_{\alpha}$ such that $\operatorname{Im} \log \Sigma_{\nu_{n}} \otimes \delta_{a\left(\nu_{n}\right)}\left(z_{0}\right)=0$. Repeating the previous arguments for the analytic functions $\log \Sigma_{\nu_{n} \bowtie \delta_{a\left(\nu_{n}\right)}}(z)$ on $\mathbb{D}_{\alpha}$, we see that $\log \Sigma_{\nu_{n} \boxplus \delta_{a\left(\nu_{n}\right)}}(z) \rightarrow 0$ uniformly for $\left|z-z_{0}\right| \leqslant \varepsilon / 4$ as $n \rightarrow \infty$. Hence $\Sigma_{\nu_{n} \bowtie \delta_{a\left(\nu_{n}\right)}}(z) \rightarrow 1$ uniformly for $\left|z-z_{0}\right| \leqslant \varepsilon / 4$ as $n \rightarrow \infty$. Recalling the definition of $\Sigma_{\nu_{n}} \otimes \delta_{a\left(\nu_{n}\right)}(z)$, we conclude from this relation that $Q_{\nu_{n} \otimes \delta_{a\left(\nu_{n}\right)}}(z) \rightarrow z$ uniformly for $\left|z-z_{0}\right| \leqslant \varepsilon / 8$ as $n \rightarrow \infty$. Since for $\mu \in \mathcal{S}_{*}$ the function $Q_{\mu}(z) / z$ is analytic and $\left|Q_{\mu}(z) / z\right| \leqslant 1$ for $z \in \mathbb{D}$, we conclude, by Vitali's theorem, that $Q_{\nu_{n}} \otimes \delta_{a\left(\nu_{n}\right)}(z) \rightarrow z$ uniformly for $z \in \mathbb{D}_{\alpha^{\prime}}$ for any $\alpha^{\prime} \in(\alpha, 1)$. This implies at once the assertion for $\left(\mathcal{M}_{*}, \bigotimes\right)$.

Thus, Proposition 6.6 is proved.

For any fixed $\gamma \in \mathcal{G}$ denote by $\left(\mathbf{M}^{\gamma} / E, \circ, L^{*}, D^{\gamma}\right)$ the $L^{*}$-metrizable semigroup of equivalence classes $\mu^{*} \in \mathbf{M}^{\gamma} / E$ with respect to the operation $\circ$ and equipped the homomorphism $D^{\gamma}$ introduced above. This semigroup is commutative, has an identity element $e:=\delta_{a}^{*}$, where $a=0,1,1$ for the semigroups $\left.(\mathcal{M}, \boxplus), \mathcal{M}_{+}, \otimes\right)$, $\left(\mathcal{M}_{*}, \nabla\right)$, respectively.

Proposition 6.7. For each $\gamma \in \mathcal{G},\left(\mathbf{M}^{\gamma} / E, \circ, L^{*}, D^{\gamma}\right)$ is Delphic.

Proof. It is sufficient to prove that assumptions (1)-(8) of Definition 3.14 hold for the semigroup $\left(\mathbf{M}^{\gamma} / E, \circ, L^{*}, D^{\gamma}\right)$.

It is easy to see that assumptions (1)-(3) hold, by Proposition 3.11, Proposition 3.12, and Proposition 3.13. Assumptions (4) and (7) hold, by Proposition 6.4 and Proposition 6.5, respectively.

By Proposition 3.11, Proposition 3.12, and Proposition 3.13, it is clear that the homomorphism $D^{\gamma}$ of $\mathbf{M}^{\gamma} / E$ into $\mathbb{R}_{+}$under addition satisfies assumption (5).

We shall verify assumption (6). At first we shall establish it for the semigroup $(\mathcal{M}, \boxplus)$. Let $D^{(\alpha, \beta)}\left(\mu^{*}\right)=0$. Then $D^{(\alpha, \beta)}(\mu)=-\operatorname{Im} \varphi_{\mu}(i(\beta+1))=0$, where 
$\mu^{*}=E \mu$. Since the function $-\operatorname{Im} \varphi_{\mu}(z)$ is harmonic and nonnegative in $\Gamma_{\alpha, \beta}$ and the point $i(\beta+1)$ lies in $\Gamma_{\alpha, \beta}$, we conclude, by minimum principle for harmonic functions, that $\operatorname{Im} \varphi_{\mu}(z)=0$ as $z \in \Gamma_{\alpha, \beta}$. From this it follows that there exists a real number $a$ such that $\varphi_{\mu}(z)=a$ as $z \in \Gamma_{\alpha, \beta}$ and we have $F_{\mu}(z)=z-a, z \in$ $\mathbb{C}^{+}$. Hence $\mu=\delta_{a}$ and the assertion is proved.

For the other semigroups $\left(\mathcal{M}_{+}, \nabla\right)$ and $\left(\mathcal{M}_{*}, \nabla\right)$ the proof of assumption (6) is similar.

It remains to show that assumption (8) holds.

Let $\left\{\mu_{j, s}^{*}: 1 \leqslant s \leqslant j<\infty\right\}$ be a triangular array in $\mathbf{M}^{\gamma} / E$ such that

$$
\lim _{j \rightarrow \infty} \max _{1 \leqslant s \leqslant j} D^{\gamma}\left(\mu_{j, s}^{*}\right)=0 \text {, and }\left\{\mu_{j}^{*}\right\} \rightarrow \mu^{*} \in \mathbf{M}^{\gamma} / E \text {, where } \mu_{j}^{*}=\mu_{j, 1}^{*} \circ \cdots \circ \mu_{j, j}^{*} \text {. }
$$

Let $k \in \mathbb{N}$ be given. Since $\max _{1 \leqslant s \leqslant j} D^{\gamma}\left(\mu_{j, s}^{*}\right) \rightarrow 0$ as $j \rightarrow \infty$ and, by Propositions 3.11 3.13. $D^{\gamma}\left(\mu_{j}^{*}\right) \rightarrow D^{\gamma}\left(\mu^{*}\right)$ as $j \rightarrow \infty$, given $n \in \mathbb{N}$ there exists $j(n)$ so large that we may group the p-measures $\mu_{j(n), s}^{*}, 1 \leqslant s \leqslant j(n)$, via free convolutions into $\nu_{l, k, n}^{*}$ say, $1 \leqslant l \leqslant k$, such that $\mu_{j(n)}^{*}=\nu_{1, k, n}^{*} \circ \cdots \circ \nu_{k, k, n}^{*}$ and $\left|D^{\gamma}\left(\nu_{l, k, n}^{*}\right)-D^{\gamma}\left(\mu^{*}\right) / k\right| \leqslant 1 / n$ for $1 \leqslant l \leqslant k$. By Proposition 6.5 , there is a decomposition $\mu^{*}=\nu_{1, k}^{*} \circ \cdots \circ \nu_{k, k}^{*}$ with $D^{\gamma}\left(\nu_{l, k}^{*}\right)=D^{\gamma}\left(\mu^{*}\right) / k$ for $1 \leqslant l \leqslant k$. Hence we may assume, without loss of generality, that there is a decomposition $\mu=\nu_{1, k} \circ \cdots \circ \nu_{k, k}$ for every $k \in \mathbb{N}$ such that $\nu_{l, k} \in \mathbf{M}^{\gamma}, a(\mu)=0$ and $a\left(\nu_{l, k}\right)=0$ for $1 \leqslant l \leqslant k$. Applying Proposition 6.6 to the sequence $\left\{\nu_{l, k}\right\}$ with $l=1, \ldots, k$ and $k=1, \ldots$, we see that $\mu \in \mathbf{M}^{\gamma}$ with any $\gamma \in \mathcal{G}$. Hence $\mu \in \mathbf{M}^{0}$ and, by Proposition $6.2, \mu^{*}$ is an i.d. element of $\mathbf{M}^{0} / E$. Therefore $\mu^{*}$ is i.d. in $\mathbf{M}^{\gamma} / E$ as desired.

Proposition 6.8. Let $\left\{\mu_{n k}: n \geqslant 1,1 \leqslant k \leqslant n\right\}$ be an array of infinitesimal measures in $\mathbf{M}^{\gamma}$. Then, for every fixed $\gamma \in \mathcal{G}, \max _{1 \leqslant k \leqslant n} D^{\gamma}\left(\mu_{n k}^{*}\right) \rightarrow 0$ as $n \rightarrow \infty$.

This proposition follows from obvious calculations which we omit.

The commutative metric semigroup $(\mathbf{M} / E$, o $)$ is covered by the union of the semigroups $\left(\mathbf{M}^{\gamma} / E, \circ, L^{*}, D^{\gamma}\right)$ as $\gamma$ runs through the set of parameters $\mathcal{G}$. By Proposition 6.3, each $\left(\mathbf{M}^{\gamma} / E, \circ, L^{*}, D^{\gamma}\right)$ is hereditary in $\mathbf{M} / E$ and is now seen to be Delphic. Thus all assumptions of Proposition 3.17 hold. Applying Proposition 3.17 to $\mathbf{M} / E$ and $\left(\mathbf{M}^{\gamma} / E, \circ, L^{*}, D^{\gamma}\right), \gamma \in \mathcal{G}$, we conclude that Theorem 3.15 holds for the semigroups $(\mathbf{M} / E, \circ)$. This result immediately implies that Theorem 2.11 and Theorem 2.12 hold.

Let us now prove Theorem 2.10. Note that $\left(\mathbf{M}^{\gamma} / E, \circ, L^{*}, D^{\gamma}\right)$ is open in $(\mathbf{M} / E, \circ$ ) (by the definition of the compact-open topology, generated on $\mathbf{M} / E$ by $L^{*}$ ) for all $\gamma \in \mathcal{G}$. In view of Proposition 6.7, Proposition 6.8, and Proposition 3.18, we obtain Khintchine's limit theorem for $(\mathbf{M} / E$, o), i.e., Theorem 2.10.

We shall now give a new proof of formulae (2.18), (2.21), and (2.24). This follows from Proposition 6.2 and from the following assertion.

Proposition 6.9. The relation $I(\mathbf{M})=\mathbf{M}^{0}$ holds. 
Proof. For every fixed $\gamma \in \mathcal{G}$, denote by $\mathbf{T}^{\gamma}$ the set of elements of $\mathbf{M}^{\gamma} / E$ which can be represented as the limit of a triangular array in $\mathbf{M}^{\gamma} / E$. As we saw in the proof of Proposition 6.7 (the proof of the assumption (8)), $\mathbf{T}^{\gamma} \subseteq \mathbf{M}^{0} / E$ for every fixed $\gamma \in \mathcal{G}$. By this relation, by Theorem 3.15, (1), and and by Proposition 6.2, we conclude that $I\left(\mathbf{M}^{0} / E\right) \subseteq I\left(\mathbf{M}^{\gamma} / E\right) \subseteq \mathbf{T}^{\gamma} \subseteq \mathbf{M}^{0} / E \subseteq I\left(\mathbf{M}^{0} / E\right)$. Hence, for every fixed $\gamma \in \mathcal{G}, I\left(\mathbf{M}^{\gamma} / E\right)=\mathbf{M}^{0} / E$. Using Proposition 6.3 we see that $I(\mathbf{M} / E)=\cup_{\gamma \in \mathcal{G}} I\left(\mathbf{M}^{\gamma} / E\right)$. Therefore $I(\mathbf{M} / E)=\mathbf{M}^{0} / E$ and we finally have $I(\mathbf{M})=\mathbf{M}^{0}$, that was to be proved.

\section{Description of the Class $I_{0}$ IN $(\mathbf{M}, \circ)$ AND NONUNiqueness of THE REPRESENTATION (2.27)}

Our next step is to prove Theorem 2.13 for the semigroups $(\mathbf{M}, \circ)$.

Proof of Theorem 2.13. Since $I_{0}$ is the set of i.d. elements all of whose factors are i.d., we should prove that any nontrivial i.d. element in $(\mathbf{M}, \circ)$ has a non i.d. factor.

7.1. Consider the case of $(\mathcal{M}, \boxplus)$. As we saw in Section 2 a measure $\mu \in \mathcal{M}$ is $\boxplus$-i.d. if and only if $\varphi_{\mu}$ admits a representation (2.18).

Let $a<b$ be real numbers such that $\nu([a, b])>0$. Consider the function

$$
\phi(z):=\varepsilon_{0} \int_{[a, b]} \frac{\left(1+u^{2}\right) \nu(d u)}{z-u}, \quad z \in \mathbb{C}^{+},
$$

with sufficiently small $\varepsilon_{0}>0$, depending on $\nu, a, b$ only, and introduce the functions $f_{j}(z):=2 \phi(z)+(-1)^{j} \varepsilon \phi^{2}(z), j=1,2$. In order to prove the theorem we need the following lemma.

Lemma 7.1. For sufficiently small $\varepsilon>0$, there exist $\mu_{j} \in \mathcal{M}, j=1,2$, such that $f_{j}(z)=\varphi_{\mu_{j}}(z)$ for $z \in \mathbb{C}^{+}$.

Proof. Consider the function

$$
F(z):=z+\phi(z), \quad z \in \mathbb{C}^{+} .
$$

By Lemma 4.1, $F: \mathbb{C}^{+} \rightarrow \mathbb{C}$ takes every value in $\mathbb{C}^{+}$precisely once. The inverse function $F^{(-1)}: \mathbb{C}^{+} \rightarrow \mathbb{C}^{+}$thus defined is in the class $\mathcal{F}$.

For any real $\eta$ denote by $\mathbb{C}_{\eta}^{+}$the half-plane $\operatorname{Im} z>\eta$. Consider the domains $\Omega_{\eta}:=F^{(-1)}\left(\mathbb{C}_{\eta}^{+}\right), \eta \in(0,1]$. Note that $\Omega_{\eta}$ are domains such that $\Omega_{\eta} \subset \mathbb{C}^{+}$.

The boundary of $\Omega_{\eta}$ is a curve $\gamma_{5}(\eta)$ characterized by the functional equation $\operatorname{Im} F(x+i y)=\eta, x \in \mathbb{R}, y>0$. Using (17.1) rewrite this equation in the form

$$
y\left(1-\varepsilon_{0} \int_{[a, b]} \frac{\left(1+u^{2}\right) \nu(d u)}{(u-x)^{2}+y^{2}}\right)=\eta .
$$

Since $F^{\prime}(z) \neq 0$ for $z \in \Omega_{\eta}, \eta>0$, we conclude, by the implicit function theorem, that $\gamma_{5}(\eta), \eta>0$, are smooth Jordan curves. 
In view of (7.2), we see that the curves $\gamma_{5}(\eta)$ lie in the union of domains $|z-(a+b) / 2|<b-a, \operatorname{Im} z>0$ and $0<\operatorname{Im} z<2 \eta$. Denote by $\tilde{\gamma}_{5}(\eta)$ the part of the curve $\gamma_{5}(\eta)$ contained in the disk $|z-(a+b) / 2| \leqslant b-a$. Let us show that

$$
\sup _{0<\eta \leqslant 1} \sup _{z \in \tilde{\gamma}_{5}(\eta)}|\phi(z)|:=A_{1}<\infty \text {. }
$$

Assume that $A_{1}=\infty$. Then for any $N>1$ there exist $\eta_{0} \in(0,1]$ and $z_{0}=$ $z_{0}\left(\eta_{0}\right) \in \tilde{\gamma}_{5}\left(\eta_{0}\right)$ such that $\operatorname{Im} F\left(z_{0}\right)>0$ and $\left|F\left(z_{0}\right)\right|>N$. Since $|\phi(z)| \rightarrow 0$ for $|z|=R \rightarrow \infty$, we easily see, by Rouché's theorem, that for $w \in \mathbb{C}^{+}$with sufficiently large modulus the equation $w=F(z)$ has a solution $z=z(w) \in \mathbb{C}^{+}$ such that $z(w)=w(1+o(1))$. Recalling that $F(z): \mathbb{C}^{+} \rightarrow \mathbb{C}$ takes every value in $\mathbb{C}^{+}$precisely once, we conclude that $z_{0}=F\left(z_{0}\right)(1+o(1))$, a contradiction for sufficiently large $N$.

Now let us show for $j=1,2$ that $F_{j}(z):=z+f_{j}(z): \mathbb{C}^{+} \rightarrow \mathbb{C}$ takes every value in $\mathbb{C}^{+}$precisely once in $F^{(-1)}\left(\mathbb{C}^{+}\right)$. By $(17.3)$, we see that for $z \in \gamma_{5}(\eta), \eta \in(0,1]$ and $\varepsilon>0$ sufficiently small the inequality $1+2(-1)^{j} \varepsilon \operatorname{Re} \phi(z)>0$ holds. Since $\operatorname{Im} \phi(z) \leqslant 0$ and $\operatorname{Im} F(z)=\eta$ for the same $z$, we conclude from the formula

$$
\operatorname{Im} F_{j}(z)=\operatorname{Im} z+\phi(z)+\operatorname{Im} \phi(z)\left(1+2(-1)^{j} \varepsilon \operatorname{Re} \phi(z)\right)
$$

that $\operatorname{Im} F_{j}(z) \leqslant \eta$ for $z \in \gamma_{5}(\eta), \eta \in(0,1]$.

Choose $R>1$ to be determined later sufficiently large. Denote by $\tilde{a}$, Re $\tilde{a}<0$ and $\tilde{b}$, Re $\tilde{b}>0$ points of intersection of the curve $\gamma_{5}(\eta)$ with the circle $|z|=R$. Consider the closed rectifiable curve $\gamma_{6}=\gamma_{6}(\eta), \eta \in(0,1]$, consisting of $\gamma_{6,1}$ : the part of $\gamma_{5}(\eta)$ lying in the disc $|z| \leqslant R$, connecting $\tilde{a}$ to $\tilde{b}$, and the arc $\gamma_{6,2}$ : $R e^{i \theta}, \arg \tilde{b}<\theta<\arg \tilde{a}$, connecting $\tilde{b}$ to $\tilde{a}$.

Fix $w \in \mathbb{C}^{+}$. Assume that $\eta<\operatorname{Im} w$. If $z$ traverses $\gamma_{6,1}$, the image $\zeta=F_{j}(z)$ lies in the half-plane $\operatorname{Im} \zeta \leqslant \eta$. If $z$ traverses $\gamma_{6,2}$, the image $\zeta=F_{j}(z)$ is equal to $\operatorname{Re}^{i \theta}(1+o(1))$ as $R \rightarrow \infty$. We conclude that the image $\zeta=F_{j}(z)$ winds around $w$ once when $z$ runs through $\gamma_{6}$ in the counter clockwise direction. By the argument principle, the function $F_{j}(z)$ takes the value $w$ precisely once inside $\gamma_{6}$. Since this assertion holds for all sufficiently large $R>1$ and for all sufficiently small $\eta>0$, we obtain the desired result.

Thus we conclude that $F_{j}^{(-1)}: \mathbb{C}^{+} \rightarrow \mathbb{C}^{+}, j=1,2$, exist and are analytic functions. Hence $F_{j}^{(-1)} \in \mathcal{N}$. Moreover it is easy to see that $F_{j}^{(-1)} \in \mathcal{F}$, by proving the lemma.

We shall complete the proof of the theorem for the $\operatorname{semigroup}(\mathcal{M}, \boxplus)$.

Since $\varphi_{\mu}(z)$ admits the representation (2.18), we may write $\mu$ in the form $\mu=\mu_{1} \boxplus \mu_{2} \boxplus \mu_{3}$, where p-measures $\mu_{1}, \mu_{2}$, and $\mu_{3}$ are determined as follows. The measures $\mu_{1}, \mu_{2}$ are defined in Lemma 7.1. Since, by (7.1),

$$
\varphi_{\mu_{1}}(z)+\varphi_{\mu_{2}}(z)=4 \phi(z)=4 \varepsilon_{0} \int_{[a, b]} \frac{1+u z}{z-u} \nu(d u)-4 \varepsilon_{0} \int_{[a, b]} u \nu(d u), \quad z \in \mathbb{C}^{+}
$$


and $\varphi_{\mu}(z)=\varphi_{\mu_{1}}(z)+\varphi_{\mu_{2}}(z)+\varphi_{\mu_{3}}(z)$, we easily conclude that

$$
\varphi_{\mu_{3}}(z):=\alpha+4 \varepsilon_{0} \int_{[a, b]} u \nu(d u)+\left(1-4 \varepsilon_{0}\right) \int_{[a, b]} \frac{1+u z}{z-u} \nu(d u)+\int_{\mathbb{R} \backslash[a, b]} \frac{1+u z}{z-u} \nu(d u) .
$$

By the Bercovici and Voiculescu result [4, the measure $\mu_{3}$ is i.d. for $\varepsilon_{0} \leqslant 1 / 4$.

If $\nu(\{a\})>0$, then we choose $b=a$ in (7.1). If $\nu(\{a\})=0$ for all $a \in \mathbb{R}$, then we choose the points $a<b$ such that $\nu([a, a+h])>c_{0} h$ and $\nu([b-h, b])>c_{0} h$ for all $0<h \leqslant h_{0}$, where $c_{0}>0$ and $h_{0}>0$ depend on the measure $\nu$ only. Such points exist by Proposition 3.6. Let us show that under these assumptions the function $\phi(z)$ (see (7.1) ), has the property

$$
(7.5)-\operatorname{Re} \phi(a-h+i h) \rightarrow+\infty, \quad h \downarrow 0, \quad \text { and } \quad \operatorname{Re} \phi(b+h+i h) \rightarrow+\infty, \quad h \downarrow 0 .
$$

This property is obvious in the case where $a=b$ and $\mu(\{a\})>0$. Consider the case where $\mu(\{a\})=0$ for all $a \in \mathbb{R}$. In this case we obtain for small $h>0$ the estimates

$$
\begin{aligned}
-\operatorname{Re} \phi(a-h+i h) & =\varepsilon_{0} \int_{[a, b]} \frac{(u-a+h)\left(1+u^{2}\right) \nu(d u)}{(u-a+h)^{2}+h^{2}} \geqslant \frac{\varepsilon_{0}}{2} \int_{[a, b]} \frac{\nu(d u)}{u-a+h} \\
& =\frac{\varepsilon_{0}}{2} \frac{\nu([a, b])}{b-a+h}+\frac{\varepsilon_{0}}{2} \int_{a}^{b} \nu([a, u)) \frac{d u}{(u-a+h)^{2}} \\
& \geqslant \frac{c_{0} \varepsilon_{0}}{2} \int_{a}^{\min \left\{a+h_{0}, b\right\}}(u-a) \frac{d u}{(u-a+h)^{2}} \geqslant-\frac{c_{0} \varepsilon_{0}}{4} \log h .
\end{aligned}
$$

This lower bound implies the first assertion of (7.5). In the same way we obtain the second assertion of (7.5).

Note that the measures $\mu_{j}, j=1,2$, are not i.d. Indeed, since

$$
\operatorname{Im} \varphi_{\mu_{j}}(z)=2 \operatorname{Im} \phi(z)\left(1+(-1)^{j} \varepsilon \operatorname{Re} \phi(z)\right)
$$

and $\operatorname{Im} \phi(z)<0, z \in \mathbb{C}^{+}$, we see from (7.5) that there exist points in $\mathbb{C}^{+}$where the functions $\operatorname{Im} \varphi_{\mu_{1}}$ and $\operatorname{Im} \varphi_{\mu_{2}}$ have positive values.

Hence the measure $\mu$ has a non-i.d. divisor and $\mu$ does not belong to the class $I_{0}$.

7.2. Let us consider the semigroup $\left(\mathcal{M}_{+}, \nabla\right)$. In order to prove Theorem 2.13 for this semigroup we need the following auxiliary lemmas.

Lemma 7.2. For every $c>0$, there exist $\mu_{j} \in \mathcal{M}_{+}, j=1,2$, such that

$$
\Sigma_{\mu_{j}}(z)=\exp \left\{-\frac{c z}{2}+(-1)^{j} i \sqrt{c z}\right\}, \quad z \in \mathbb{C}^{+} .
$$

Here and in the sequel we shall choose the principle branch of $\sqrt{z}$. 
Proof. In the first step we consider the function

$$
w=g_{1}(z):=\log z-\frac{c z}{2}-i \sqrt{c z} . \quad z \in \mathbb{C}^{+} .
$$

Let us show that for every fixed $w \in \mathbb{C}^{+}$such that $0<\operatorname{Im} w<\pi$ there exists an unique $z \in \mathbb{C}^{+}$such that $w=g_{1}(z)$. To prove this assertion we consider the closed rectifiable curve $\gamma_{7}$, consisting of the interval $\gamma_{7,1}: t,-R \leqslant t \leqslant-1 / R$, connecting $-R$ to $-1 / R$, the arc $\gamma_{7,2}: e^{i \varphi} / R, 0<\varphi<\pi$, connecting $-1 / R$ to $1 / R$, the interval $\gamma_{7,3}: t, 1 / R \leqslant t \leqslant R$, connecting $1 / R$ to $R$, and the arc $\gamma_{7,4}: R e^{i \varphi}, 0<\varphi<\pi$, connecting $R$ to $-R$. The parameter $R \geqslant 1$ will be chosen later on sufficiently large.

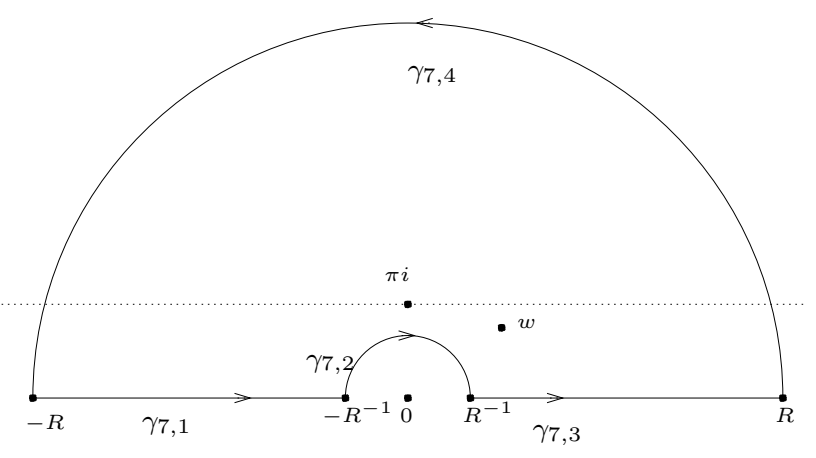

FiguRE 5 .

Let $z$ traverse $\gamma_{7}$ in the counter clockwise direction.

If $z$ traverses $\gamma_{7,1}$ the image $g_{1}\left(\gamma_{7,1}\right)$ is contained in the line $\operatorname{Im} \zeta=\pi$, connecting $\log R+c R / 2+\sqrt{c R}+i \pi$ to $-\log R+c /(2 R)+\sqrt{c / R}+i \pi$.

If $z$ traverses $\gamma_{7,2}$ the image $g_{1}\left(\gamma_{7,2}\right)$ is contained in the half-plane $\operatorname{Re} \zeta \leqslant$ $-\log R+1$, connecting $-\log R+c /(2 R)+\sqrt{c / R}+i \pi$ to $-\log R-c /(2 R)-i \sqrt{c / R}$.

If $z$ traverses $\gamma_{7,3}$ the image $\zeta=g_{1}\left(\gamma_{7,3}\right)$ is contained in the half-plane $\operatorname{Im} \zeta<0$, connecting $-\log R-c /(2 R)-i \sqrt{c / R}$ to $\log R-c R / 2-i \sqrt{c R}$.

Finally, if $z$ traverses $\gamma_{7,4}$ the image $\zeta=g_{1}\left(\gamma_{7,4}\right)$ is contained in the domain $\{|\zeta| \geqslant c R / 2, \operatorname{Im} \zeta \leqslant \pi\}$, connecting the points $\log R-c R / 2-i \sqrt{c R}$ to $\log R+$ $c R / 2+\sqrt{c R}+i \pi$.

Therefore we can conclude that the image $\zeta=g_{1}(z), z \in \gamma_{7}$, winds around $w$ once when $z$ runs through $\gamma_{7}$. Hence there is a unique point $z$ inside the curve $\gamma_{7}$ such that $w=g_{1}(z)$. Thus we have proved that for every fixed $w \in \mathbb{S}_{\pi}=$ $\{w \in \mathbb{C}: 0<\operatorname{Im} w<\pi\}$ there exists an unique $z \in \mathbb{C}^{+}$such that (7.6) holds. Thus the inverse function $g_{1}^{(-1)}: \mathbb{S}_{\pi} \rightarrow \mathbb{C}^{+}$exists and is analytic in $\mathbb{S}_{\pi}$. Introduce the function $R_{1}(z):=g_{1}^{(-1)}(\log z), z \in \mathbb{C}^{+}$. We note that $R_{1} \in \mathcal{N}$ and $R_{1}^{(-1)}(z)=$ $z \exp \{-c z / 2-i \sqrt{c z}\}$ in the domain of $\mathbb{C}^{+}$, where $R_{1}^{(-1)}$ is uniquely defined.

Let us show that $R_{1}$ admits an analytic continuation on $(-\infty, 0)$ and its value on $(-\infty, 0)$ is negative. Moreover $R_{1}(x) \rightarrow 0$ as $x \uparrow 0$. It is easy to see that 
$\left(e^{g_{1}(x)}\right)^{\prime}>0$ for $x<0$. Since $e^{g_{1}(z)}$ is analytic on $(-\infty, 0)$, we conclude that $\left(e^{g_{1}}\right)^{(-1)}$ exists and is analytic on $(-\infty, 0)$ as well. This function coincides for $z \in \mathbb{C}^{+}$with the function $R_{1}(z)$ defined earlier. From the definition of $e^{g_{1}(z)}$ it follows that $\left(e^{g_{1}}\right)^{(-1)}(x)<0$ for $x<0$ and $\left(e^{g_{1}}\right)^{(-1)}(x) \rightarrow 0$ as $x \uparrow 0$. By Corollary 3.3, $R_{1}(z)$ belongs to the class $\mathcal{K}$. Therefore there exists $\mu_{1} \in \mathcal{M}_{+}$such that $R_{\mu_{1}}(z)=R_{1}(z)$ for $z \in \mathbb{C}^{+}$and we proved the assertion of the lemma in the case $j=1$.

Now we shall consider the function

$$
w=g_{2}(z):=\log z-\frac{c z}{2}+i \sqrt{c z} . \quad z \in \mathbb{C}^{+} .
$$

Let us show that for every fixed $w \in \mathbb{C}^{+}$such that $-2 \pi<\operatorname{Im} w<-\pi$ there exists an unique $z \in \mathbb{C}^{+}$such that $w=g_{2}(z)$.

To prove this assertion we consider as before the closed rectifiable curve $\gamma_{7}$ and as before $z$ traverses $\gamma_{7}$ in the counter clockwise direction.

If $z$ traverses $\gamma_{7,1}$ the image $\zeta=g_{2}\left(\gamma_{7,1}\right)$ is contained in the line $\operatorname{Im} \zeta=\pi$, connecting $\log R+c R / 2-\sqrt{c R}+i \pi$ to $-\log R+c /(2 R)-\sqrt{c / R}+i \pi$.

If $z$ traverses $\gamma_{7,2}$ the image $\zeta=g_{2}\left(\gamma_{7,2}\right)$ is contained in the half-plane $\operatorname{Re} \zeta \leqslant$ $-\log R+1$, connecting $-\log R+c /(2 R)-\sqrt{c / R}+i \pi$ to $-\log R-c /(2 R)+i \sqrt{c / R}$.

If $z$ traverses $\gamma_{7,3}$ the image $\zeta=g_{2}\left(\gamma_{7,3}\right)$ is contained in the half-plane $\operatorname{Im} \zeta>0$, connecting $-\log R-c /(2 R)+i \sqrt{c / R}$ to $\log R-c R / 2+i \sqrt{c R}$.

If $z$ traverses $\gamma_{7,4}$ the image $\zeta=g_{2}\left(\gamma_{7,4}\right)$ is contained in the domain $\{|\zeta| \geqslant$ $c R / 2,-\pi-4 / \sqrt{c R} \leqslant \arg \zeta \leqslant 4 / \sqrt{c R}$, connecting $\log R-c R / 2+i \sqrt{c R}$ to $\log R+$ $c R / 2-\sqrt{c R}+i \pi$.

Therefore we deduce that the image $\zeta=g_{2}(z)$ winds around $w$ once when $z$ runs through $\gamma_{7}$. Hence there is a unique point $z$ inside the curve $\gamma_{7}$ such that $w=g_{2}(z)$. This relation holds for all sufficiently large $R>1$. Thus we have proved that for every fixed $w \in \mathbb{S}_{-\pi}:=\{w \in \mathbb{C}:-2 \pi<\operatorname{Im} w<-\pi\}$ there exists an unique $z \in \mathbb{C}^{+}$such that (17.7) holds. Then we deduce that the inverse function $g_{2}^{(-1)}: \mathbb{S}_{-\pi} \rightarrow \mathbb{C}^{+}$exists and is analytic in $\mathbb{S}_{-\pi}$. Introduce the function $R_{2}(z):=g_{2}^{(-1)}(\log z-2 \pi i), z \in \mathbb{C}^{+}$. Obviously, $R_{2}^{(-1)}(z)=z \exp \{-c z / 2+i \sqrt{c z}\}$, where $R_{2}^{(-1)}$ is well defined.

As in the case of $j=1$ we show show that $R_{2}(z)$ admits an analytic continuation on $(-\infty, 0)$ and its value on $(-\infty, 0)$ is negative. Moreover $R_{2}(x) \rightarrow 0$ as $x \uparrow 0$. Hence, by Corollary 3.3, $R_{2}(z)$ belongs to the class $\mathcal{K}$. Therefore there exists $\mu_{2} \in \mathcal{M}_{+}$such that $R_{\mu_{2}}(z)=R_{2}(z)$ for $z \in \mathbb{C}^{+}$and we proved the assertion of the lemma in the case $j=2$ as well.

Lemma 7.3. For every $c>0$, there exist $\mu_{j} \in \mathcal{M}_{+}, j=1,2$, such that

$$
\Sigma_{\mu_{j}}(z)=\exp \left\{\frac{c}{2 z}+(-1)^{j} i \sqrt{\frac{c}{z}}\right\}, \quad z \in \mathbb{C}^{+} .
$$



it.

The proof of this lemma is similar to the proof of Lemma 7.2 therefore we omit

Let the functions $f_{j}(z), j=1,2$, are defined as in Lemma 7.1. In addition we assume in (7.1) that $0<a \leqslant b<\infty$.

Lemma 7.4. For sufficiently small $\varepsilon>0$, there exist $\mu_{j} \in \mathcal{M}_{+}, j=1,2$, such that

$$
\Sigma_{\mu_{j}}(z)=\exp \left\{f_{j}(z)\right\}, \quad z \in \mathbb{C}^{+} .
$$

Proof. Consider the functions

$$
G(z):=\log z+\phi(z), \quad z \in \mathbb{C}^{+} .
$$

By Lemma 5.3, $G: \mathbb{C}^{+} \rightarrow \mathbb{C}$ takes every value in $\mathbb{S}_{\pi}$ precisely once. Recall that $\mathbb{S}_{\pi}:=\{z \in \mathbb{C}: 0<\operatorname{Im} z<\pi\}$. For any $\eta \in(0,1 / 10)$ denote by $\mathbb{S}_{\eta}$ the strip $\{z \in \mathbb{C}: \eta<\operatorname{Im} z<\pi-\eta\}$. Consider the domains $\Omega_{\eta}:=G^{(-1)}\left(\mathbb{S}_{\eta}\right), \eta \in(0,1 / 10)$. Note that $\Omega_{\eta}$ is a domain such that $\Omega_{\eta} \subset \mathbb{C}^{+}$.

The boundary of $\Omega_{\eta}$ is a curve $\gamma_{8}(\eta)$ consisting of curves $\gamma_{8,1}(\eta)$ and $\gamma_{8,2}(\eta)$ characterized by the equations $\operatorname{Im} G(x+i y)=\pi-\eta, x<0, y>0$, and $\operatorname{Im} G(x+$ $i y)=\eta, x>0, y>0$, respectively. Since $G^{\prime}(z) \neq 0$ for $z \in \Omega_{\eta}$, we conclude, by the implicit function theorem, that $\gamma_{8,1}(\eta)$ and $\gamma_{8,2}(\eta), \eta \in(0,1 / 10)$, are smooth Jordan curves.

We see, by the definition of $\gamma_{8,1}(\eta)$, that this curve, connecting $\infty$ to 0 , lies in the angular region $\pi-2 \eta<\arg z<\pi$. By definition the curve $\gamma_{8,2}(\eta)$, connecting 0 to $\infty$, lies in the union of domains $|z-(a+b) / 2|<b / 2, \operatorname{Im} z>0$, and $0<\arg z<2 \eta$.

Denote by $\tilde{\gamma}_{8}(\eta)$ the part of the curve $\gamma_{8}(\eta)$, lying in the disk $|z-(a+b) / 2| \leqslant$ $b / 2$. Let us show that

$$
\sup _{0<\eta \leqslant \frac{1}{10}} \sup _{z \in \tilde{\gamma}_{8}(\eta)}|\phi(z)|:=A_{2}<\infty
$$

Assume that $A_{2}=\infty$. Then for any $N>1$ there exist $\eta_{0} \in(0,1 / 10]$ and $z_{0}=z_{0}\left(\eta_{0}\right) \in \tilde{\gamma}_{8}\left(\eta_{0}\right)$ such that $\eta<\operatorname{Im} G\left(z_{0}\right)<\pi-\eta$ and $\left|G\left(z_{0}\right)\right|>N$. Since $|\phi(z)| / \log |z| \rightarrow 0$ as $|z|=R, 1 / R$ and $R \rightarrow \infty$, we easily see, by Rouché's theorem, that for $w \in \mathbb{S}_{\pi}$ with sufficiently large modulus the equation $w=G(z)$ has a solution $z=z(w) \in \mathbb{S}_{\pi}$ such that $z(w)=\exp \{w(1+o(1))\}$. Recalling that $G(z): \mathbb{C}^{+} \rightarrow \mathbb{C}$ takes every value in $\mathbb{S}_{\pi}$ precisely once, we conclude that $z_{0}=\exp \left\{G\left(z_{0}\right)(1+o(1))\right\}$, a contradiction for sufficiently large $N$.

Now let us show for $j=1,2$ that $G_{j}(z):=\log z+f_{j}(z): \mathbb{C}^{+} \rightarrow \mathbb{C}$ takes every value in $\mathbb{S}_{\pi}$ precisely once in $G^{(-1)}\left(\mathbb{S}_{\pi}\right)$. By the formula

$$
\operatorname{Im} G_{j}(z)=\arg z+\operatorname{Im} \phi(z)+\operatorname{Im} \phi(z)\left(1+2(-1)^{j} \varepsilon \operatorname{Re} \phi(z)\right)
$$

and by (17.8), we see that $\operatorname{Im} G_{j}(z) \leqslant \eta$, where $z \in \gamma_{8,2}(\eta), \eta \in(0,1 / 10)$, and $\varepsilon>0$ is sufficiently small. 
Using (17.1), we note that, for $z \in \gamma_{8,1}(\eta), \eta \in(0,1 / 10)$,

$$
\begin{aligned}
-\operatorname{Im} \phi(z) & =\varepsilon_{0} \operatorname{Im} z \int_{[a, b]} \frac{\left(1+u^{2}\right) \nu(d u)}{(u-\operatorname{Re} z)^{2}+(\operatorname{Im} z)^{2}} \leqslant \varepsilon_{0} \frac{1+b^{2}}{a} \nu([a, b]) \frac{\operatorname{Im} z}{|\operatorname{Re} z|} \\
& \leqslant 2 \varepsilon_{0} \frac{1+b^{2}}{a} \nu([a, b]) \eta .
\end{aligned}
$$

Therefore we conclude from (17.9) that $\operatorname{Im} G_{j}(z) \geqslant \pi-2 \eta$ for $z \in \gamma_{8,1}(\eta), \eta \in$ $(0,1 / 10)$, and sufficiently small $\varepsilon_{0}, \varepsilon>0$. In addition we see from (7.1) that for $|z|=1 / R$ and $|z|=R$ the inequality $|\phi(z)| \leqslant c$ holds, with a positive constant $c$, depending on $\phi$ only, where $R>1$ is sufficiently large.

Let $R>1$ be sufficiently large. Denote by $c^{\prime}$ and $c^{\prime \prime}$ the intersection points of the curve $\gamma_{8,1}(\eta)$ with the circles $|z|=R$ and $|z|=1 / R$, respectively. We denote as well by $d^{\prime \prime}$ and $d^{\prime}$ the intersection points of the curve $\gamma_{8,2}(\eta)$ with the circles $|z|=1 / R$ and $|z|=R$, respectively.

Consider the closed rectifiable curve $\gamma_{9}=\gamma_{9}(\eta), \eta \in(0,1 / 10)$, consisting of $\gamma_{9,1}$ : the part of $\gamma_{8,1}(\eta)$ lying in the disc $|z| \leqslant R$, connecting $c^{\prime}$ to $c^{\prime \prime}$, the arc $\gamma_{9,2}$ : $e^{i \theta} / R, \arg d^{\prime \prime}<\theta<\arg c^{\prime \prime}$, connecting $c^{\prime \prime}$ to $d^{\prime \prime}$, the curve $\gamma_{9,3}$ : the part of $\gamma_{8,2}(\eta)$ lying in the disc $|z| \leqslant R$, connecting $d^{\prime \prime}$ to $d^{\prime}$, and the $\operatorname{arc} \gamma_{9,4}: R e^{i \theta}$, arg $d^{\prime}<$ $\theta<\arg c^{\prime}$, connecting $d^{\prime}$ to $c^{\prime}$.

Assume that $w \in \mathbb{S}_{\pi}$ and $\eta<\frac{1}{2} \min \{\operatorname{Im} w, \pi-\operatorname{Im} w\}$. If $z$ traverses $\gamma_{9,1}$, the image $\zeta=G_{j}(z)$ lies in the half-plane $\operatorname{Im} \zeta \geqslant \pi-2 \eta$. If $z$ traverses $\gamma_{9,2}$, it is easy to see that the image $\zeta=G_{j}(z)$ lies in the half-plane $\operatorname{Re} \zeta<-\frac{1}{2} \log R$. If $z$ traverses $\gamma_{9,3}$, the image $\zeta=G_{j}(z)$ lies in the half-plane $\operatorname{Im} \zeta \leqslant \eta$. Finally, if $z$ traverses $\gamma_{9,4}$, the image $\zeta=G_{j}(z)$, lies in the half-plane $\operatorname{Re} \zeta>\frac{1}{2} \log R$.

Summarizing, it follows that the image $\zeta=G_{j}(z)$ winds around $w$ once when $z$ traverses $\gamma_{9}$. By the argument principle, the function $G_{j}(z)$ takes the value $w$ precisely once inside $\gamma_{9}$. Since this assertion holds for all sufficiently large $R>1$ and for all sufficiently small $\eta>0$, we obtain the desired result.

This result implies that the inverse functions $\rho_{j}=G_{j}^{(-1)}: \mathbb{S}_{\pi} \rightarrow \mathbb{C}^{+}, j=1,2$, exist and are analytic on $\mathbb{S}_{\pi}$.

Let us show that $\rho_{j}(z)$ admit an analytic continuation on the half-line $\gamma_{-}$: $\operatorname{Im} z=\pi, \operatorname{Re} z<0$, and that their values on this half-line are negative. It is easy to see that

$$
G_{j}^{\prime}(x)=\frac{1}{x}+2 \phi^{\prime}(x)\left(1+(-1)^{j} \varepsilon \phi(x)\right)<0, \quad x<0, j=1,2 .
$$

Since $f_{j}(z)$ is analytic on $(-\infty, 0)$, we conclude that $G_{j}^{(-1)}$ exists and is analytic on $\gamma_{-}$as well. Since, as shown above, for every fixed $w \in \mathbb{S}_{\pi}$ there is an unique point $z \in G^{(-1)}\left(\mathbb{C}^{+}\right)$such that $w=\log z+f_{j}(z)$ holds, this function coincides for $z \in \mathbb{S}_{\pi}$ with the function $\rho_{j}(z)$ obtained early. Introduce the function $\tilde{\rho}_{j}(z):=\rho_{j}(\log z), z \in \mathbb{C}^{+}$. Note that $\tilde{\rho}_{j} \in \mathcal{N}$ and $\tilde{\rho}_{j}^{(-1)}(z)=z \exp \left\{f_{j}(z)\right\}$, 
on the domain $\mathbb{C}^{+}$, where $\tilde{\rho}_{j}^{(-1)}$ is uniquely defined. Moreover, the function $\tilde{\rho}_{j}(z)$ admits an analytic continuation on $(-\infty, 0)$.

From the definition of $\tilde{\rho}_{j}(z)$ it follows that $\tilde{\rho}_{j}(x)<0$ for $x<0$ and $\tilde{\rho}_{j}(x) \rightarrow 0$ as $x \uparrow 0$. By Corollary 3.3. $\tilde{\rho}_{j}, j=1,2$, belong to the class $\mathcal{K}$ and we conclude the assertion of the lemma.

Now we complete the proof of Theorem 2.13 for the semigroup $\left(\mathcal{M}_{+}, \bigotimes\right)$.

At first note that the measure $\mu \in \mathcal{M}_{+}$with $\Sigma_{\mu}(z)=\exp \{-c z\}$, where $c>0$, does not belong to the class $I_{0}$. Indeed, by Lemma 7.2, there exist measures $\mu_{1}$ and $\mu_{2}$ of $\mathcal{M}_{+}$such that

$$
\Sigma_{\mu_{j}}=\exp \left\{-c z / 2+(-1)^{j} i \sqrt{c z}\right\}, \quad z \in \mathbb{C}^{+}, \quad j=1,2 .
$$

It is easy to see that $\operatorname{Im}\left(-c z / 2+(-1)^{j} i \sqrt{c z}\right)$ has positive values at some points of the half-plane $\mathbb{C}^{+}$. Hence $\mu_{j}, j=1,2$, are not i.d. elements in the semigroup $\left(\mathcal{M}_{+}, \otimes\right)$. On the other hand $\Sigma_{\mu}(z)=\Sigma_{\mu_{1}}(z) \Sigma_{\mu_{2}}(z)$ and we see that $\mu$ has noni.d. divisors as was to be proved. In a similar way, using Lemma 7.3, we prove that the measure $\mu \in \mathcal{M}_{+}$with $\Sigma_{\mu}(z)=\exp \{c / z\}$, where $c>0$, does not belong to the class $I_{0}$.

Now we consider the case where $\Sigma_{\mu}(z)$ admits a representation (2.21) with $a=b=0, \nu(\{0\})=0$ and $\nu((0, \infty))>0$.

For every fixed $0<a \leqslant b$, we have the representation

$$
\Sigma_{\mu}(z)=\Sigma_{\mu_{1}}(z) \Sigma_{\mu_{2}}(z) \Sigma_{\mu_{3}}(z), \quad 0<\arg z<2 \pi,
$$

where

$$
\Sigma_{\mu_{3}}(z):=\exp \left\{4 \varepsilon_{0} \int_{[a, b]} u \nu(d u)+\left(1-4 \varepsilon_{0}\right) \int_{[a, b]} \frac{1+u z}{z-u} \nu(d u)\right\}+\int_{\mathbb{R} \backslash[a, b]} \frac{1+u z}{z-u} \nu(d u)
$$

and $\Sigma_{\mu_{j}}(z), j=1,2$, are defined in Lemma 7.4.

If $\nu(\{a\})>0$, then we assume in (17.1) $a=b$. If $\nu(\{a\})=0$ for all $a \in(0, \infty)$, then we choose the points $0<a<b$ so that such that $\nu([a, a+h])>c h$ and $\nu([b-$ $h, b])>c h$ for all $0<h \leqslant h_{0}$, where $c>0$ and $h_{0}>0$ depend on the measure $\nu$ only. Such points exist by Proposition 3.6. We showed in Section 7.1 that under these assumptions the function $\phi(z)$, see (7.1), has the limiting behaviour (17.5).

Note that the measures $\mu_{j}, j=1,2$, are not i.d. Indeed, by (17.5), there exist points in $\mathbb{C}^{+}$at which the functions $\operatorname{Im} \log \Sigma_{\mu_{1}}$ and $\operatorname{Im} \log \Sigma_{\mu_{2}}$ have positive values. Hence the measure $\mu$ has a non-i.d. divisor and $\mu$ does not belong to the class $I_{0}$.

7.3. It remains to prove Theorem 2.13 for the semigroup $\left(\mathcal{M}_{*}, \nabla\right)$. We need the following auxiliary result.

Lemma 7.5. For sufficiently small $\varepsilon>0$, there exist $\mu_{j} \in \mathcal{M}_{*}, j=1,2$, such that

$$
\Sigma_{\mu_{j}}(z)=\exp \left\{q_{j}(z)\right\}, \quad z \in \mathbb{D}
$$


where $q_{j}(z):=2 q(z)+(-1)^{j} i \varepsilon q^{2}(z), j=1,2$, and, for $\xi_{0} \in \mathbb{T}$ and $0<\Delta<1 / 100$,

$$
q(z):=\varepsilon_{0} \int_{\left\{\xi \in \mathbb{T}:\left|\xi-\xi_{0}\right|<\Delta\right\}} \frac{\xi+z}{\xi-z} \nu(d \xi), \quad z \in \mathbb{D} .
$$

In $7.10 \varepsilon_{0}>0$ is a sufficiently small constant, depending on $\nu$ and $\Delta$ only, and $\nu\left(\left\{\xi \in \mathbb{T}:\left|\xi-\xi_{0}\right|<\Delta\right\}\right)>0$.

Proof. Let us prove the lemma in the case $j=1$. One can prove the lemma in the case $j=2$ in the same way. Without loss of generality we assume that in the definition of the function $q(z)$ the parameter $\xi_{0}$ is equal to 1 .

Consider the function

$$
Q(z):=\log z+q(z), \quad z \in \tilde{\mathbb{D}}:=\mathbb{D} \backslash[-1,0]
$$

This function is analytic on $\tilde{\mathbb{D}}$.

Denote by $\gamma_{10,1}, \gamma_{10,2}$, and $\gamma_{10,3}$ the Jordan curves defined by the parametric equations $\zeta=\log (1-t)+i \pi+q(t-1), 0 \leqslant t<1, \zeta=i t+q(-1),-\pi \leqslant t \leqslant \pi$, and $\zeta=\log t-i \pi+q(-t), 0<t \leqslant 1$, respectively. Note that $\operatorname{Re} q(-1)=0$. Define a curvilinear half-strip $S_{-}$as a domain with boundary $\gamma_{10,1}, \gamma_{10,2}$, and $\gamma_{10,3}$. Let us show that $Q: \tilde{\mathbb{D}} \rightarrow \mathbb{C}$ takes every value in $S_{-}$precisely once. For this we need to prove that for any $w \in S_{-}$there exists an unique point $z \in \tilde{\mathbb{D}}$ such that $w=Q(z)$, provided that $\varepsilon_{0}>0$ is sufficiently small.

Consider the contour $\gamma_{3}$ with parameter $\theta=\pi$ (see Section 5). Choose $r \in$ $(0,1)$ such that $1-r$ is small. If $z$ traverses $\gamma_{3,1}$ the image $\zeta=Q(z)$ lies on the curve $\gamma_{10,1}$. If $z$ traverses $\gamma_{3,2}$ the image $\zeta=Q(z)$ lies in the half-plane $\operatorname{Re} \zeta<\frac{1}{2} \log (1-r)$. If $z$ traverses $\gamma_{3,3}$ the image $\zeta=Q(z)$ lies on the curve $\gamma_{10,3}$. Finally, by the inequality $\operatorname{Re} q(z) \geqslant 0, z \in \mathbb{D}$, if $z$ traverses $\gamma_{3,4}$ the image $\zeta=Q(z)$ lies in the half-plane $\operatorname{Re} \zeta>\log r$.

Hence, the image $\zeta=Q(z)$ winds around $w$ once when $z$ runs through $\gamma_{3}$ in the counter clockwise direction. By the argument principle, the function $Q(z)$ takes the value $w \in S_{-}$precisely once inside $\gamma_{3}$ for sufficiently small $1-r$ and the desired result is proved. Hence the inverse function $Q^{(-1)}: S_{-} \rightarrow \tilde{\mathbb{D}}$ is defined and is analytic function on $S_{-}$.

Let $\gamma_{10,2}(\eta)$ is the half-open interval of the vertical line $\operatorname{Re} z=-\eta, \eta \in(0,1 / 100)$, lying between the curves $\gamma_{10,1}$ and $\gamma_{10,3}$. Denote by $\gamma_{11}(\eta)$ the closed rectifiable Jordan curve $Q^{(-1)}\left(\gamma_{10,2}(\eta)\right)$. By the definition of $\gamma_{11}(\eta)$, we note that $\operatorname{Re} Q(z)=-\eta$ for $z \in \gamma_{11}(\eta), \eta \in(0,1 / 100)$. Since $Q: \tilde{\mathbb{D}} \rightarrow \mathbb{C}$ takes every value in $S_{-}$precisely once, we note that if $z$ traverses the curve $\gamma_{11}(\eta)$ in the counter clockwise direction, then $\operatorname{Im} Q(z)$ is a monotone function such that $-\pi+\alpha(-\eta) \leqslant$ $\operatorname{Im} Q(z) \leqslant \pi+\alpha(-\eta)$, where, as it is easy to see, $|\alpha(-\eta)-q(-1)| \leqslant 1 / 10$ for $\eta \in(0,1 / 100)$. 
Let us prove

$$
\sup _{0<\eta \leqslant \frac{1}{100}} \sup _{z \in \gamma_{11}(\eta)}|q(z)| \leqslant 1 / 3 .
$$

We shall assume that $\eta \in(0,1 / 100)$. Note that $|q(z)| \leqslant 1 / 10$ for $z \in \gamma_{11}(\eta) \cap$ $\{|z-1| \geqslant 2 \Delta\}$. From the relation $\operatorname{Re} Q(z)=-\eta, z \in \gamma_{11}(\eta)$, and the inequality $\log |z| \geqslant \log (1-2 \Delta)$ for $z \in \gamma_{11}(\eta) \cap\{|z-1|<2 \Delta\}$ we see that for such $z$ the bound $|\operatorname{Re} q(z)| \leqslant 1 / 10$ holds. In addition it is not difficult to see that the inequality $\left|Q\left(z^{\prime}\right)\right| \leqslant 1 / 10$ holds at points of intersection $z^{\prime}$ of $\gamma_{11}(\eta)$ with the circle $|z-1|=2 \Delta$. Since $\operatorname{Im} Q(z), z \in \gamma_{11}(\eta)$, is a monotone function, we have $|\operatorname{Im} Q(z)| \leqslant 1 / 10$ and hence $|\operatorname{Im} q(z)| \leqslant-\log (1-2 \Delta)+\arctan (2 \Delta)+1 / 10 \leqslant 1 / 5$ for $z \in \gamma_{11}(\eta) \cap\{|z-1|<2 \Delta\}$. Thus (7.11) is proved.

Then, by the relation

$$
\log |z|+\operatorname{Re} q_{1}(z)=\operatorname{Re} Q(z)+\operatorname{Re} q(z)(1+2 \varepsilon \operatorname{Im} q(z))
$$

and (7.11), we conclude that

$$
\log |z|+\operatorname{Re} q_{1}(z) \geqslant-\eta, z \in \gamma_{11}(\eta), \eta \in(0,1 / 100) .
$$

Now we shall prove that $\tilde{q}_{1}(z):=z e^{q_{1}(z)}: \mathbb{D} \rightarrow \mathbb{C}$ takes every value $w \in \mathbb{D}$ precisely once in $Q^{(-1)}\left(S_{-}\right) \cup(-1,0]$.

Fix $w \in \mathbb{D}$. Let $\eta>0$ be sufficiently small. If $z$ traverses $\gamma_{11}(\eta)$ in the counter clockwise direction we see, by (7.11) and (17.12), that $\left|\operatorname{Arge}^{q_{1}(z)}\right| \leqslant 1 / 2<\pi$ and $\left|e^{q_{1}(z)}\right| \geqslant e^{-\eta}$, respectively. By the argument principle, the function $z e^{q_{1}(z)}$ takes the value $w \in \mathbb{D}$ precisely once inside $\gamma_{11}(\eta)$ for sufficiently small $\eta>0$. We obtain the desired result.

Hence the inverse function $\tilde{q}_{1}^{(-1)}: \mathbb{D} \rightarrow \mathbb{D}$ thus defined is analytic on $\mathbb{D}$ and, as it is easy to see, belongs to the class $\mathcal{S}_{*}$. The lemma is proved.

Let us complete the proof of Theorem 2.13 for the semigroup $\left(\mathcal{M}_{*}, \otimes\right)$. Without loss of generality, we assume that $\nu(A)>0$, where $A:=\{\xi \in \mathbb{T},|\xi-1|<\Delta\}$ and write

$$
\Sigma_{\mu}(z)=\Sigma_{\mu_{1}}(z) \Sigma_{\mu_{2}}(z) \Sigma_{\mu_{3}}(z), \quad z \in \mathbb{D}
$$

where

$$
\Sigma_{\mu_{3}}(z):=\left(1-4 \varepsilon_{0}\right) \int_{A} \frac{\xi+z}{\xi-z} \nu(d \xi)+\int_{\mathbb{T} \backslash A} \frac{\xi+z}{\xi-z} \nu(d \xi)
$$

and $\Sigma_{j}(z), j=1,2$, are defined in Lemma 7.5. If $\nu(\{1\}>0$, we assume $A:=\{1\}$. If $\nu(\{\xi\})=0$ for all $\xi \in \mathbb{T}$, we choose the point $\xi_{1} \in \mathbb{T}, \xi_{1} \neq 1$, so that $\nu(\{\xi \in$ $\left.\mathbb{T},\left|\xi-\xi_{1}\right|<h\right\}>c h$ for all $0<h \leqslant h_{0}$, where the constant $c>0$ and $h_{0}>0$ depend on the measure $\nu$ only. By Proposition 3.6, such points exist. Repeating the arguments used in the proof of (17.5), we obtain

$$
\operatorname{Im} q\left(\xi_{1} e^{i h}(1-h)\right) \rightarrow+\infty, \quad h \uparrow 1 .
$$


Note that the measures $\mu_{j}, j=1,2$ are not i.d. Indeed, by (7.13), there exist points $z \in \mathbb{D}$ where the modulus of the functions $\Sigma_{\mu_{1}}(z)$ is strictly less than 1 . Hence the measure $\mu$ has non-i.d. divisors and therefore $\mu \notin I_{0}$.

Now we shall prove that there exists a measure $\mu \in \mathbf{M}$ for which the representation (2.27) is not unique. We establish this result for the semigroup $(\mathcal{M}, \boxplus)$. One can prove this fact for the semigroups $\left(\mathcal{M}_{+}, \nabla\right)$ and $\left(\mathcal{M}_{*}, \nabla\right)$ in the same way.

Assume to the contrary that every measure $\mu \in \mathcal{M}$ admits an unique representation

$$
\mu=\mu_{1} \boxplus \mu_{2} \ldots,
$$

where $\mu_{1}, \mu_{2}, \ldots$ are some indecomposable nondegenerate elements of the semi$\operatorname{group}(\mathcal{M}, \boxplus)$, with respect to the equivalence relation $\mu \sim \nu$ if $\mu=\nu \boxplus \delta_{a}$ for some $a \in \mathbb{R}$.

Let $\mu$ be an i.d. p-measure. Hence, for every $n \in \mathbb{N}, \mu=\nu_{n} \boxplus \cdots \boxplus \nu_{n}(n$ times), where $\nu_{n} \in \mathcal{M}$. Since the p-measure $\nu_{n}$ admits an unique representation of the form (7.14), we see that, for every $n \in \mathbb{N}, \mu=\mu_{1}^{n \boxplus} \boxplus \rho_{n}$, where $\mu_{1}$ denotes the measure from representation (7.14) and $\rho_{n} \in \mathcal{M}$. We return to the notation of Section 6. Note that the measure $\mu$ belongs to the semigroup $\mathcal{M}^{(\alpha, \beta)}$ for some $\alpha>0$ and $\beta>0$. Hence $-\operatorname{Im} \varphi_{\mu}(i(\beta+1)) \geqslant 0$. By Proposition 6.3, $-\operatorname{Im} \varphi_{\mu_{1}^{n \boxplus}}(i(\beta+1)) \geqslant 0$ and $-\operatorname{Im} \varphi_{\rho_{n}}(i(\beta+1)) \geqslant 0$ as well. Since

$$
\begin{aligned}
\operatorname{Im} \varphi_{\mu}(i(\beta+1)) & \left.=\operatorname{Im} \varphi_{\mu_{1}^{n \boxplus}}(i(\beta+1))\right)+\operatorname{Im} \varphi_{\rho_{n}}(i(\beta+1)) \\
& \left.=n \operatorname{Im} \varphi_{\mu_{1}}(i(\beta+1))\right)+\operatorname{Im} \varphi_{\rho_{n}}(i(\beta+1)),
\end{aligned}
$$

we have $\operatorname{Im} \varphi_{\mu_{1}}(i(\beta+1)) \rightarrow 0$ as $n \rightarrow \infty$ and we get $\operatorname{Im} \varphi_{\mu_{1}}(i(\beta+1))=0$. This immediately implies that $\operatorname{Im} \varphi_{\mu_{1}}(z)=0$ for $z \in \Gamma_{\alpha, \beta}$ and $\mu_{1}=\delta_{a}$ for some $a \in \mathbb{R}$. We arrive at a contradiction which proves the assertion.

\section{Dense Classes of indecomposable elements in $(\mathbf{M}, \circ)$}

In this section we prove Theorem 2.14.

8.1. At first we shall prove this theorem for the semigroup $(\mathcal{M}, \boxplus)$.

Assume that $\mu \in \mathcal{M}$ has finite support. Then the Cauchy transform $G_{\mu}(z)$ is a rational function such that $\lim _{z \rightarrow \infty} z G_{\mu}(z)=1$. Therefore $F_{\mu}(z)$ is a rational function as well and $\lim _{z \rightarrow \infty}\left(F_{\mu}(z) / z\right)=1$. Since $F_{\mu} \in \mathcal{F}$, it admits the representation

$$
F_{\mu}(z)=z+c+\sum_{k=1}^{l} \frac{a_{k}}{t_{k}-z}, \quad z \in \mathbb{C}^{+},
$$

where $c \in \mathbb{R}$ and $a_{k}>0, k=1, \ldots, l$, and $t_{1}<t_{2}<\cdots<t_{l}$. We assume without loss of generality that $c=0$. Let $\mu_{j} \in \mathcal{M}, j=1,2$, and $\mu=\mu_{1} \boxplus \mu_{2}$. Let us prove that either $\mu_{1}=\mu \boxplus \delta_{a}, \mu_{2}=\delta_{-a}$ or $\mu_{1}=\delta_{-a}, \mu_{2}=\mu \boxplus \delta_{a}$ with $a \in \mathbb{R}$. By Theorem 2.1, there exist functions $Z_{j}(z) \in \mathcal{F}, j=1,2$, such that (2.3) holds 
and $F_{\mu}(z)=F_{\mu_{1}}\left(Z_{1}(z)\right)=F_{\mu_{2}}\left(Z_{2}(z)\right), z \in \mathbb{C}^{+}$. Using the integral representation (3.3) for Nevanlinna functions, we rewrite the last relation in the form

$$
\begin{aligned}
z+\sum_{k=1}^{l} \frac{a_{k}}{t_{k}-z} & =b_{j}+Z_{j}(z)+\int_{-\infty}^{\infty}\left(\frac{1}{t-Z_{j}(z)}-\frac{t}{1+t^{2}}\right) \sigma_{j}(d t) \\
& :=b_{j}+Z_{j}(z)+W_{j}(z), \quad j=1,2, \quad z \in \mathbb{C}^{+},
\end{aligned}
$$

where $b_{j} \in \mathbb{R}$ and $\sigma_{j}$ are nonnegative measures such that $\int_{\mathbb{R}} \sigma_{j}(d t) /\left(1+t^{2}\right)<\infty$. Since, again by representation (3.3) for functions in $\mathcal{F}$, we have

$$
Z_{j}(z)=z+c_{j}+\int_{-\infty}^{\infty}\left(\frac{1}{t-z}-\frac{t}{1+t^{2}}\right) \nu_{j}(d t), j=1,2, \quad z \in \mathbb{C}^{+},
$$

where $c_{j} \in \mathbb{R}$ and $\nu_{j}$ are nonnegative measures such that $\int_{\mathbb{R}} \nu_{j}(d t) /\left(1+t^{2}\right)<\infty$. Moreover, by (3.4), $\left|Z_{j}(i y)-i y\right|=o(y), j=1,2$, as $y \rightarrow+\infty$. We note that the functions $W_{j}(z) \in \mathcal{N}, j=1,2$, and, as it is easy to see, $\left|W_{j}(i y)\right| / y \rightarrow$ $0, j=1,2$, as $y \rightarrow+\infty$. Therefore, by (3.3) and (3.4), $W_{j}(z), j=1,2$, admit the representation

$$
W_{j}(z)=\tilde{c}_{j}+\int_{-\infty}^{\infty}\left(\frac{1}{t-z}-\frac{t}{1+t^{2}}\right) \tilde{\sigma}_{j}(d t), \quad z \in \mathbb{C}^{+},
$$

where $\tilde{c}_{j} \in \mathbb{R}$ and $\tilde{\sigma}_{j}$ are nonnegative measures such that $\int_{\mathbb{R}} \tilde{\sigma}_{j}(d t) /\left(1+t^{2}\right)<\infty$. Hence, we finally obtain, for $z \in \mathbb{C}^{+}$and $j=1,2$,

$$
\sum_{k=1}^{l} \frac{a_{k}}{t_{k}-z}=b_{j}+c_{j}+\tilde{c}_{j}+\int_{-\infty}^{\infty}\left(\frac{1}{t-z}-\frac{t}{1+t^{2}}\right)\left(\nu_{j}+\tilde{\sigma}_{j}\right)(d t) .
$$

Applying Stieltjes inversion formula (3.7) to (8.1), we see that the measures $\nu_{j}, j=1,2$, have finite supports which are contained in the set $\left\{t_{k}\right\}_{k=1}^{l}$. Hence either $Z_{j}(z)=z+c_{j}$ or

$$
Z_{j}(z)=z+c_{j}+\sum_{s=1}^{m_{j}} \frac{d_{s, j}}{\rho_{s, j}-z}
$$

where $c_{j} \in \mathbb{R}, d_{s, j}>0, s=1, \ldots, m_{j}$, and $\rho_{1, j}<\cdots<\rho_{m_{j}, j}$. In (8.2) $\left\{\rho_{s, j}\right\}_{s=1}^{m_{j}} \subseteq$ $\left\{t_{k}\right\}_{k=1}^{l}, j=1,2$.

Let one of the $Z_{j}$, say $Z_{1}$, be of the form $Z_{1}(z)=z+c_{1}$, then $F_{\mu_{1}}(z)=F_{\mu}\left(z-c_{1}\right)$ and we have $\mu_{1}=\mu \boxplus \delta_{c_{1}}$ and $\varphi_{\mu_{1}}(z)=\varphi_{\mu}(z)+c_{1}$. From relation (2.4) $\varphi_{\mu}(z)=$ $\varphi_{\mu_{1}}(z)+\varphi_{\mu_{2}}(z)$, we obtain $\varphi_{\mu_{2}}(z)=-c_{1}$ which implies $\mu_{2}=\delta_{-c_{1}}$.

It remains to consider the case where both $Z_{1}$ and $Z_{2}$ have the form (8.2). By the relation (2.3), we obtain

$$
z=Z_{1}(z)+Z_{2}(z)-F_{\mu_{1}}\left(Z_{1}(z)\right)=Z_{1}(z)+Z_{2}(z)-F_{\mu}(z), \quad z \in \mathbb{C}^{+} .
$$


This together with (8.2) yields, using $c_{1}+c_{2}=0$ as $z \rightarrow \infty$,

$$
\sum_{k=1}^{l} \frac{a_{k}}{t_{k}-z}=\sum_{s=1}^{m_{1}} \frac{d_{s, 1}}{\rho_{s, 1}-z}+\sum_{s=1}^{m_{2}} \frac{d_{s, 2}}{\rho_{s, 2}-z}, \quad z \in \mathbb{C}^{+} .
$$

This implies that either $\rho_{1,1}=t_{1}$ or $\rho_{1,2}=t_{1}$. Let us assume for definiteness that $\rho_{1,1}=t_{1}$.

First we shall show that $\sigma_{1}$ has finite support. Let $\rho_{0,1}:=-\infty$ and $\rho_{m_{1}+1,1}:=$ $+\infty$. Note that $Z_{1}^{\prime}(x)>0$ for $x \in \mathbb{R} \backslash\left\{\rho_{1,1}, \ldots, \rho_{m_{1}, 1}\right\}$. Furthermore, $x \downarrow \rho_{s, 1}$ implies $Z_{1}(x) \downarrow-\infty$ for $s=0,1, \ldots, m_{1}$ and $x \uparrow \rho_{s, 1}$ implies $Z_{1}(x) \uparrow+\infty$ for $s=1, \ldots, m_{1}+1$. Since $Z_{1}(z) \in \mathcal{F}$ the function $1 /\left(t-Z_{1}(z)\right)$ is in $\mathcal{N}$ and $z /\left(t-Z_{1}(z)\right)$ converges to 1 as $z \rightarrow \infty$ for any fixed $t \in \mathbb{R}$. In view of these properties we conclude that the Nevanlinna integral representation (3.3) for the functions $1 /\left(t-Z_{1}(z)\right), t \in \mathbb{R}$, has the form

$$
\frac{1}{t-Z_{1}(z)}=\sum_{q=1}^{m_{1}+1} \frac{\alpha_{q}(t)}{\beta_{q}(t)-z}
$$

where $\alpha_{q}(t)>0, q=1, \ldots, m_{1}+1$, and $\beta_{1}(t)<\rho_{1,1}<\beta_{2}(t)<\rho_{2,1}<\cdots<$ $\beta_{m}(t)<\rho_{m_{1}, 1}<\beta_{m_{1}+1}(t)$. Moreover $\alpha_{q}(t), q=1, \ldots, m_{1}+1$, are continuous functions and $\beta_{q}(t), q=1, \ldots, m_{1}+1$, are strictly increasing functions. In addition, it is easy to see, for $t<-t_{0}$, where $t_{0}>0$ is sufficiently large, $1 / 2 \leqslant \alpha_{1}(t) \leqslant 3 / 2$ and $\left|\beta_{1}(t)-t-b_{3}\right| \leqslant 1$, where $b_{3} \in \mathbb{R}$. For $t>t_{0}$ we obtain $1 / 2 \leqslant \alpha_{m_{1}+1}(t) \leqslant 3 / 2$ and $\left|\beta_{m_{1}+1}(t)-t-b_{3}\right| \leqslant 1$. With the help of these relations we deduce from (8.1), (8.3), and the Stieltjes inversion formula that the measure $\sigma_{1}$ has bounded support. Using again (8.1) and (8.3), we conclude that

$$
\sum_{q=1}^{m_{1}+1} \int_{-\infty}^{\infty} \frac{\alpha_{q}(t) \sigma_{1}(d t)}{\beta_{q}(t)-z}=\sum_{k=1}^{l} \frac{a_{k}^{\prime}}{t_{k}-z}, \quad z \in \mathbb{C}^{+},
$$

where $a_{k}^{\prime} \geqslant 0$. Since $\alpha_{1}(t)>0, t \in \mathbb{R}$, and $\beta_{1}(t)<\rho_{1,1}=t_{1}$, we see that the first summand on the left hand-side of (8.4) can be written in the form

$$
\int_{-\infty}^{\infty} \frac{\alpha_{1}(t) \sigma_{1}(d t)}{\beta_{1}(t)-z}=\int_{\left(-\infty, t_{1}\right)} \frac{\alpha_{1}\left(\beta_{1}^{(-1)}(u)\right) \tilde{\sigma}_{1}(d u)}{u-z},
$$

where $\tilde{\sigma}_{1}$ is a measure such that $\tilde{\sigma}_{1}(B):=\sigma_{1}\left(\beta_{1}^{(-1)}(B)\right)$ for any Borel set $B$. Note that $\tilde{\sigma}_{1}\left(\left(-\infty, t_{1}\right)\right)=\sigma_{1}(\mathbb{R})$. Applying to both sides of (8.4) the inversion formula (3.4), we obtain that $\sigma_{1}(\mathbb{R})=0$. Hence relation (8.4) implies that $a_{k}^{\prime}=0$ for all $k$ and $\sigma_{1} \equiv 0$. Thus, $F_{\mu_{1}}(z)=z+b_{1}$ and $\mu_{1}=\delta_{-b_{1}}$. Hence $\mu_{2}=\mu \boxplus \delta_{b_{1}}$. This implies the theorem.

8.2. We shall now prove Theorem 2.14 for the semigroup $\left(\mathcal{M}_{+}, \nabla\right)$. Let $\mu_{1}, \mu_{2}$ denote measures in $\mathcal{M}_{+}$and assume that $\mu:=\mu_{1} \otimes \mu_{2}$ has finite support $S$. 
Without loss of generality we assume that $S=\left\{u_{1}, \ldots, u_{l}\right\}$, where $0<u_{1}<$ $\cdots<u_{l}$. If the support has the form $S=\left\{0, u_{1}, \ldots, u_{l}\right\}$, the proof is similar. We have to prove that either $\mu_{1}=\mu \otimes \delta_{a}$ and $\mu_{2}=\delta_{1 / a}$ or $\mu_{1}=\delta_{1 / a}$ and $\mu_{2}=\mu \otimes \delta_{a}$, where $a \in(0,+\infty)$. By Theorem 2.4, there exist functions $Z_{j}(z) \in \mathcal{K}, j=1,2$, such that $\psi_{\mu}(z)=\psi_{\mu_{1}}\left(Z_{1}(z)\right)=\psi_{\mu_{2}}\left(Z_{2}(z)\right)$ for $z \in \mathbb{C}^{+}$and

$$
Z_{1}(z) Z_{2}(z)=z R_{\mu_{j}}\left(Z_{j}(z)\right)=z \psi_{\mu_{j}}\left(Z_{j}(z)\right) /\left(1+\psi_{\mu_{j}}\left(Z_{j}(z)\right)\right), \quad z \in \mathbb{C}^{+}, \quad j=1,2 .
$$

Rewrite the first of these relations in the form

$$
\sum_{k=1}^{l} p_{k} \frac{z u_{k}}{1-z u_{k}}=\int_{\mathbb{R}_{+}} \frac{Z_{j}(z) u}{1-Z_{j}(z) u} \mu_{j}(d u), \quad z \in \mathbb{C}^{+}, \quad j=1,2,
$$

where $p_{k}>0, k=1, \ldots, l$, and $p_{1}+\cdots+p_{l}=1$. Recalling the definition of the Krein class $\mathcal{K}$, note that the functions

$$
K_{j}(z ; u)=\frac{Z_{j}(z) u}{1-Z_{j}(z) u}, \quad z \in \mathbb{C}^{+}, \quad j=1,2,
$$

belong to the class $\mathcal{K}$ for every fixed $u>0$. Therefore they admit the representation

$$
K_{j}(z ; u)=a_{j}(u) z+z \int_{(0, \infty)} \frac{\tau_{u, j}(d t)}{t-z}, \quad z \in \mathbb{C}^{+}, \quad u>0, \quad j=1,2,
$$

where $a_{j}(u) \geqslant 0$ and $\tau_{u, j}$ is a nonnegative measure, satisfying condition (2.9). It is easy to see that $a_{j}(u)=0$. Moreover the functions $u \mapsto \tau_{u, j}$ are continuous. Using the previous representation for $K_{j}(z ; u)$, we easily deduce from (8.6) the relations

$$
\sum_{k=1}^{l} p_{k} \frac{z u_{k}}{1-z u_{k}}=\int_{\mathbb{R}_{+}} K_{j}(z ; u) \mu_{j}(d u)=z \int_{(0, \infty)} \frac{\nu_{j}(d t)}{t-z}, \quad z \in \mathbb{C}^{+}, \quad j=1,2
$$

where $\nu_{j}(B):=\int_{\mathbb{R}_{+}} \tau_{u, j}(B) \mu_{j}(d u)$ for every Borel set $B$ in $\mathbb{R}_{+}$. By (8.7), the measures $\nu_{j}$ have finite support which coincides with the set $S_{1}:=\left\{1 / u_{k}\right\}_{k=1}^{l}$. Hence, it follows that, for every point $u \in(0,+\infty)$ such that

$$
\mu_{j}((u-h, u+h))>0
$$

for any $h>0$, the measure $\tau_{u, j}$ has finite support which is contained in $S_{1}$. Hence we obtain, for points $u$ satisfying (8.8),

$$
K_{j}(z ; u)=\frac{Z_{j}(z) u}{1-Z_{j}(z) u}=\sum_{k=1}^{l} p_{k, j}(u) \frac{z u_{k}}{1-z u_{k}},
$$

where $p_{k, j}(u) \geqslant 0, k=1, \ldots, l$. Let us show that the number $N_{j}$ of points $u$ satisfying (8.8) does not exceed $l$. Assume to the contrary that $N_{j}>l$. Then there exist at least two points $u^{\prime}, u^{\prime \prime} \in(0,+\infty), u^{\prime} \neq u^{\prime \prime}$, such that in (8.9) 
$p_{k, j}\left(u^{\prime}\right)>0, p_{k, j}\left(u^{\prime \prime}\right)>0$ for some $k \in\{1, \ldots, l\}$. Therefore we may conclude from (8.9) that $Z_{j}\left(r+1 / u_{k}\right) \rightarrow 1 / u^{\prime}$ and $Z_{j}\left(r+1 / u_{k}\right) \rightarrow 1 / u^{\prime \prime}$ as $r \downarrow 0$. Since $u^{\prime} \neq u^{\prime \prime}$, this is impossible and we arrive at a contradiction. Let us assume that $1<N_{j}<l, j=1,2$. Rewrite (8.7) in the form

$$
\sum_{k=1}^{l} p_{k} \frac{z u_{k}}{1-z u_{k}}=\sum_{m=1}^{N_{j}} q_{m, j} K_{j}\left(z ; u_{j}^{(m)}\right)
$$

where $0<u_{j}^{(1)}<\cdots<u_{j}^{\left(N_{j}\right)}$ are points, satisfying (8.8), and therefore $q_{m, j}>$ $0, m=1, \ldots, N_{j}$. Return to (8.9) and let $u=u_{j}^{(m)}$. From the arguments above it follows that there does not exist an index $k$ such that the coefficients $p_{k, j}\left(u_{j}^{(m)}\right)$ in the representation (8.9) are positive for two different indices $m^{\prime} \neq m^{\prime \prime}$. Denote by $N_{j}(u)$ the number of summands on the right-hand side of (8.9).

We have two cases. Either for some $j=1,2$, say $j=1$, there exist at least two points $u_{1}^{\left(m^{\prime}\right)}$ and $u_{1}^{\left(m^{\prime \prime}\right)}$ such that $N_{1}\left(u_{1}^{\left(m^{\prime}\right)}\right) \neq N_{1}\left(u_{1}^{\left(m^{\prime \prime}\right)}\right)$ or $N_{j}\left(u_{j}^{(1)}\right)=\cdots=$ $N_{j}\left(u_{j}^{\left(N_{j}\right)}\right)=N_{j}^{*}$ for $j=1,2$.

Consider the first case. Let us assume for definiteness that $N_{1}\left(u_{1}^{\left(m^{\prime}\right)}\right)<N_{1}\left(u_{1}^{\left(m^{\prime \prime}\right)}\right)$. Rewriting (8.9) we obtain $Z_{1}(z)=K_{1}(z ; u) /\left(u\left(K_{1}(z ; u)+1\right)\right)$. Hence we arrive at the equality

$$
\frac{K_{1}\left(z ; u_{1}^{\left(m^{\prime}\right)}\right)}{u_{1}^{\left(m^{\prime}\right)}\left(K_{1}\left(z ; u_{1}^{\left(m^{\prime}\right)}\right)+1\right)}=\frac{K_{1}\left(z ; u_{1}^{\left(m^{\prime \prime}\right)}\right)}{u_{1}^{\left(m^{\prime \prime}\right)}\left(K_{1}\left(z ; u_{1}^{\left(m^{\prime \prime}\right)}\right)+1\right)}, \quad z \in \mathbb{C}^{+} .
$$

This equality implies

$$
K_{1}\left(z ; u_{1}^{\left(m^{\prime \prime}\right)}\right)=\frac{u_{1}^{\left(m^{\prime \prime}\right)} K_{1}\left(z ; u_{1}^{\left(m^{\prime}\right)}\right)}{\left(u_{1}^{\left(m^{\prime}\right)}-u_{1}^{\left(m^{\prime \prime}\right)}\right) K_{1}\left(z ; u_{1}^{\left(m^{\prime}\right)}\right)+u_{1}^{\left(m^{\prime}\right)}}, \quad z \in \mathbb{C}^{+} .
$$

It is easy to see that both sides of (8.11) are meromorphic functions and the number of poles of the function $K_{1}\left(z ; u^{\left(m^{\prime \prime}\right)}\right)$ is $N_{1}\left(u_{1}^{\left(m^{\prime \prime}\right)}\right)$, but the number of poles of the function in the right-hand side of (8.11) does not exceed $N_{1}\left(u^{\left(m^{\prime}\right)}\right)$. Thus we arrive at a contradiction. We emphasize that here and in the sequel we consider poles in $\mathbb{C}$ only.

Now we shall consider the second case. In this case we may represent $l$ as $l=N_{j} N_{j}^{*}, j=1,2$. Let $\left\{\rho_{1, m, j}, \ldots, \rho_{N_{j}^{*}, m, j}\right\}$, where $0<\rho_{1, m, j}<\cdots<\rho_{N_{j}^{*}, m, j}$, be poles of $K_{j}\left(z ; u_{j}^{(m)}\right)$. We see that $K_{j}^{\prime}\left(x ; u_{j}^{(m)}\right)>0$ for $x \in \mathbb{R} \backslash\left\{\rho_{1, m, j}, \ldots, \rho_{N_{j}^{*}, m, j}\right\}$. Furthermore, $x \downarrow \rho_{s, m, j}$ implies $K_{j}\left(x ; u_{j}^{(m)}\right) \downarrow-\infty$ for $s=1, \ldots, N_{j}^{*}$ and $x \uparrow \rho_{s, m, j}$ implies $K_{j}\left(x ; u_{j}^{(m)}\right) \uparrow+\infty$ for $s=1, \ldots, N_{j}^{*}$. In addition $K_{j}\left(x ; u_{j}^{(m)}\right) \downarrow-1$ if $x \downarrow-\infty$ and $K_{j}\left(x ; u_{j}^{(m)}\right) \uparrow-1$ if $x \uparrow+\infty$.

Let us assume that one of $N_{1}$ or $N_{2}$, say $N_{1}$, is not equal to 2 . Then $N_{1}^{*} \leqslant l / 3$ and $N_{2}^{*} \leqslant l / 2$. Since $Z_{j}(z)=K_{j}(z ; u) /\left(u\left(K_{j}(z ; u)+1\right)\right)$, we note from (8.9) that the number of poles of the functions $Z_{1}(z), Z_{2}(z)$ are equal to $N_{1}^{*}-1, N_{2}^{*}-1$, 
respectively. Return to the relation (8.5). We see that the number of poles of the function $Z_{1}(z) Z_{2}(z)$ does not exceed $5 l / 6-2$, but, as it is easy to see, the number of poles of the function $R_{\mu_{1}}\left(Z_{1}(z)\right)$ is equal to $l-1$, a contradiction.

Consider the case $N_{1}=N_{2}=2$. In this case we obtain from (8.10)

$$
q_{1,1} K_{1}\left(z ; u_{1}^{(1)}\right)+q_{2,1} K_{1}\left(z ; u_{1}^{(2)}\right)=q_{1,2} K_{2}\left(z ; u_{2}^{(1)}\right)+q_{2,2} K_{2}\left(z ; u_{2}^{(2)}\right), \quad z \in \mathbb{C} .
$$

As we saw before the meromorphic functions $K_{j}\left(z ; u_{j}^{(1)}\right)$ and $K_{j}\left(z ; u_{j}^{(2)}\right)$ have no common poles and union of their poles coincides with the set of poles of the meromorphic function $\psi_{\mu}(z)$. By (8.11), the poles of $K_{1}\left(z ; u_{1}^{(1)}\right)$ coincides with the zeros of the function $K_{1}\left(z ; u_{1}^{(2)}\right)+u_{1}^{(2)} /\left(u_{1}^{(2)}-u_{1}^{(1)}\right)$. Note that $u_{1}^{(2)} /\left(u_{1}^{(2)}-u_{1}^{(1)}\right)>1$. Let $\left\{\rho_{1, j}, \ldots, \rho_{l / 2, j}\right\}$, where $0<\rho_{1, j}<\cdots<\rho_{l / 2, j}$, be poles of $K_{1}\left(z ; u_{1}^{(j)}\right)$. As shown above $K_{1}^{\prime}\left(x ; u_{1}^{(j)}\right)>0$ for $x \in \mathbb{R} \backslash\left\{\rho_{1, j}, \ldots, \rho_{l / 2, j}\right\}$. Furthermore, $x \downarrow \rho_{s, j}$ implies $K_{1}\left(x ; u_{1}^{(j)}\right) \downarrow-\infty$ for $s=1, \ldots, l / 2$ and $x \uparrow \rho_{s, j}$ implies $K_{1}\left(x ; u_{1}^{(j)}\right) \uparrow+\infty$ for $s=1, \ldots, l / 2$. In addition $K_{1}\left(x ; u_{1}^{(j)}\right) \downarrow-1$ if $x \downarrow-\infty$ and $K_{1}\left(x ; u_{1}^{(j)}\right) \uparrow-1$ if $x \uparrow+\infty$. Using this information about the behaviour of $K_{1}\left(z ; u_{1}^{(j)}\right)$, we see that the poles of the functions $K_{1}\left(z ; u_{1}^{(1)}\right)$ and $K_{1}\left(z ; u_{1}^{(2)}\right)$ alternate and $\rho_{1,1}>\rho_{1,2}$. A similar result holds for the functions $K_{2}\left(z ; u_{2}^{(j)}\right), j=1,2$. Hence we conclude that for $k=1,2$ the poles of $K_{1}\left(z ; u_{1}^{(k)}\right)$ coincide with the poles of $K_{2}\left(z ; u_{2}^{(k)}\right)$. Now we deduce from (8.12) that

$$
q_{1,1} K_{1}\left(z ; u_{1}^{(1)}\right)=q_{1,2} K_{2}\left(z ; u_{2}^{(1)}\right) \quad \text { and } \quad q_{2,1} K_{1}\left(z ; u_{1}^{(2)}\right)=q_{2,2} K_{2}\left(z ; u_{2}^{(2)}\right), \quad z \in \mathbb{C} \text {. }
$$

The first of these relations implies that

$$
Z_{1}(z)=q_{1,2} \frac{u_{2}^{(1)}}{u_{1}^{(1)}} \frac{Z_{2}(z)}{q_{1,1}+\left(q_{1,2}-q_{1,1}\right) u_{2}^{(1)} Z_{2}(z)}, \quad z \in \mathbb{C} .
$$

Therefore relation (8.5) may be written as

$$
\frac{\alpha Z_{2}^{2}(z)}{\beta+\gamma Z_{2}(z)}=z R_{\mu_{1}}\left(Z_{1}(z)\right)=z \frac{\psi_{\mu_{1}}\left(Z_{1}(z)\right)}{1+\psi_{\mu_{1}}\left(Z_{1}(z)\right)}, \quad z \in \mathbb{C},
$$

where $\alpha, \beta>0$ and $\gamma \in \mathbb{R}$. Note that zeros of the function $R_{\mu_{1}}\left(Z_{1}(z)\right)$ coincide with the zeros of $\psi_{\mu_{1}}\left(Z_{1}(z)\right)$ and are thereby simple. But the left-hand side of (8.13) implies that there exist multiple zeros of the function $\psi_{\mu_{1}}\left(Z_{1}(z)\right)$, a contradiction.

Hence for one of $j=1,2$, say $j=1$, we have $N_{1}=1$ or $N_{1}=l$. Consider the case $N_{1}=l$. In this case the relation (8.9) for $j=1$ has the form

$$
K_{1}\left(z ; u_{1}^{(m)}\right)=\frac{Z_{1}(z) u_{1}^{(m)}}{1-Z_{1}(z) u_{1}^{(m)}}=p_{k(m), 1}\left(u_{1}^{(m)}\right) \frac{z u_{k(m)}}{1-z u_{k(m)}}, \quad m=1, \ldots, l .
$$

where $k\left(m^{\prime}\right) \neq k\left(m^{\prime \prime}\right)$ if $m^{\prime} \neq m^{\prime \prime}$. From (8.6) we conclude that $Z_{1}(x) \rightarrow-\infty$ as $x \rightarrow-\infty$ and which in (8.14) implies $p_{k(m), 1}\left(u_{1}^{(m)}\right)=1, m=1, \ldots, l$. Hence it follows from (8.14) that $Z_{1}(z)=z u_{k(m)} / u^{(m)}$ for all $m=1, \ldots, l$. Thus $Z_{1}(z)=$ 
$z u_{0}$ for some $u_{0} \in(0,+\infty)$ and we arrive at $\psi_{\mu}(z)=\psi_{\mu_{1}}\left(z u_{0}\right), z \in \mathbb{C}^{+}$. We finally conclude that $\mu=\mu_{1} \otimes \delta_{u_{0}}$. This means that $\mu_{1}=\mu_{\bigotimes} \delta_{1 / u_{0}}$ and $\mu_{2}=\delta_{u_{0}}$.

It remains to consider the case $N_{1}=1$. Here we easily see that $\mu_{1}=\delta_{u_{0}}$ for some $u_{0} \in(0,+\infty)$ and hence $\mu_{2}=\mu_{\square} \delta_{1 / u_{0}}$.

Thus, Theorem 2.14 is completely proved for the semigroup $\left(\mathcal{M}_{+}, \bigotimes\right)$.

8.3. Let us prove Theorem 2.14 for the semigroup $\left(\mathcal{M}_{*}, \nabla\right)$. The proof is similar to the proof of Theorem 2.14 for the semigroup $\left(\mathcal{M}_{+}, \bigotimes\right)$. For convenience of the reader we include it.

Let $\mu_{1}, \mu_{2}, \in \mathcal{M}_{*}$ and let $\mu:=\mu_{1} \otimes \mu_{2}$ have the finite support $\left\{\xi_{1}, \ldots, \xi_{l}\right\}$. We have to prove that either $\mu_{1}=\mu \otimes \delta_{a}$ and $\mu_{2}=\delta_{1 / a}$ or $\mu_{1}=\delta_{1 / a}$ and $\mu_{2}=\mu \otimes \delta_{a}$, where $a \in \mathbb{T}$. By Theorem 2.7, there exists a function $Z_{1}(z) \in \mathcal{S}_{*}$ such that $\psi_{\mu}(z)=\psi_{\mu_{1}}\left(Z_{1}(z)\right)=\psi_{\mu_{2}}\left(Z_{2}(z)\right)$ for $z \in \mathbb{D}$ and

(8.15) $Z_{1}(z) Z_{2}(z)=z Q_{\mu_{j}}(z)=z \psi_{j}\left(Z_{j}(z)\right) /\left(1+\psi_{j}\left(Z_{j}(z)\right)\right), \quad z \in \mathbb{D}, \quad j=1,2$.

Rewrite the first of these relations in the form

$$
\sum_{k=1}^{l} p_{k} \frac{z \xi_{k}}{1-z \xi_{k}}=\int_{\mathbb{T}} \frac{Z_{j}(z) \xi}{1-Z_{j}(z) \xi} \mu_{j}(d \xi), \quad z \in \mathbb{D}, \quad j=1,2,
$$

where $p_{k}>0, k=1, \ldots, l, p_{1}+\cdots+p_{l}=1$, and $\xi_{k} \in \mathbb{T}, k=1, \ldots, l$. Since the functions

$$
C_{j}(z ; \xi)=\frac{1+Z_{j}(z) \xi}{1-Z_{j}(z) \xi}, \quad z \in \mathbb{D}, \quad j=1,2,
$$

belong to the Carathéodory class $\mathcal{C}$ for every fixed $\xi \in \mathbb{T}$, they admit the representation

$$
C_{j}(z ; \xi)=\int_{\mathbb{T}} \frac{\zeta+z}{\zeta-z} \sigma_{\xi, j}(d \zeta), \quad z \in \mathbb{D}, \quad j=1,2,
$$

where $\sigma_{\xi, j}$ are p-measures. Moreover for $j=1,2$ the functions $\xi \mapsto \sigma_{\xi, j}$ are continuous. From (8.16) we deduce the relations

$$
\sum_{k=1}^{l} p_{k} \frac{1+z \xi_{k}}{1-z \xi_{k}}=\int_{\mathbb{T}} C_{j}(z ; \xi) \mu_{j}(d \xi)=\int_{\mathbb{T}} \frac{\zeta+z}{\zeta-z} \nu_{j}(d \zeta), \quad z \in \mathbb{D},
$$

where $\nu_{j}(B):=\int_{\mathbb{T}} \sigma_{\xi, j}(B) \mu_{j}(d \xi)$ for every Borel set $B$ of $\mathbb{T}$. As above, by (8.17), the measures $\nu_{j}$ have finite support which coincides with the set $S:=\left\{1 / \xi_{k}\right\}_{k=1}^{l}$. Hence, for every point $\zeta \in \mathbb{T}$ such that

$$
\mu_{j}(\{\xi \in \mathbb{T}: \arg \zeta-h<\arg \xi<\arg \zeta+h\})>0
$$

for any $h>0, \sigma_{\xi, j}$ has finite support which is contained in $S$. Thus we obtain, for such points $\xi$,

$$
C_{j}(z ; \xi)=\frac{1+Z_{j}(z) \xi}{1-Z_{j}(z) \xi}=\sum_{k=1}^{l} p_{k, j}(\xi) \frac{1+z \xi_{k}}{1-z \xi_{k}}
$$


where $p_{k, j}(\xi) \geqslant 0, k=1, \ldots, l$. We shall show that the number $N_{j}$ of points $\xi$ satisfying (8.18) does not exceed $l$. Assume to the contrary that $N_{j}>l$. Then there exist at least two points $\xi^{\prime}, \xi^{\prime \prime} \in \mathbb{T}, \xi^{\prime} \neq \xi^{\prime \prime}$, such that in (8.19) $p_{k, j}\left(\xi^{\prime}\right)>0, p_{k, j}\left(\xi^{\prime \prime}\right)>0$ for some $k \in\{1, \ldots, l\}$. Therefore we can conclude from (8.19) that $Z_{j}\left(r / \xi_{k}\right) \rightarrow 1 / \xi^{\prime}$ and $Z_{j}\left(r / \xi_{k}\right) \rightarrow 1 / \xi^{\prime \prime}$ as $r \rightarrow 1$. Since $\xi^{\prime} \neq \xi^{\prime \prime}$, this is impossible and we arrive at a contradiction. Let us assume that $1<N_{j}<l, j=$ 1,2 . Rewrite (8.17) in the form

$$
\sum_{k=1}^{l} p_{k} \frac{1+z \xi_{k}}{1-z \xi_{k}}=\sum_{m=1}^{N_{j}} q_{m, j} C_{j}\left(z ; \xi_{j}^{(m)}\right)
$$

where $\xi_{j}^{(m)} \in \mathbb{T}$ are points, satisfying (8.18), and therefore $q_{m, j}>0, m=$ $1, \ldots, N_{j}$. Return to (8.19) with $\xi=\xi_{j}^{(m)}$. Note as above that there does not exist an index $k$ such that the coefficients satisfy $p_{k, j}\left(\xi^{(m)}\right)>0$ in the representation (8.19) for two different indices $m^{\prime} \neq m^{\prime \prime}$. Denote by $N_{j}(\xi)$ the number of summands in the right-hand side of (8.19).

Again we have two cases. Either for some $j=1,2$, say $j=1$, there exist at least two points $\xi_{1}^{\left(m^{\prime}\right)}$ and $\xi_{1}^{\left(m^{\prime \prime}\right)}$ such that $N_{1}\left(\xi_{1}^{\left(m^{\prime}\right)}\right) \neq N_{1}\left(\xi_{1}^{\left(m^{\prime \prime}\right)}\right)$ or $N_{j}\left(\xi_{j}^{(1)}\right)=$ $\cdots=N_{j}\left(\xi_{j}^{\left(N_{j}\right)}\right):=N_{j}^{*}$ for $j=1,2$.

Consider the first case. Assume for definiteness $N\left(\xi^{\left(m^{\prime}\right)}\right)<N\left(\xi^{\left(m^{\prime \prime}\right)}\right)$. From (8.19) it follows that $Z_{1}(z)=\left(C_{1}(z ; \xi)-1\right) /\left(\xi\left(C_{1}(z ; \xi)+1\right)\right)$. Hence we obtain the equality

$$
\frac{C_{1}\left(z ; \xi_{1}^{\left(m^{\prime}\right)}\right)-1}{\xi_{1}^{\left(m^{\prime}\right)}\left(C_{1}\left(z ; \xi_{1}^{\left(m^{\prime}\right)}\right)+1\right)}=\frac{C_{1}\left(z ; \xi_{1}^{\left(m^{\prime \prime}\right)}\right)-1}{\xi_{1}^{\left(m^{\prime \prime}\right)}\left(C_{1}\left(z ; \xi_{1}^{\left(m^{\prime \prime}\right)}\right)+1\right)}, \quad z \in \mathbb{D} .
$$

This equality implies

$$
C_{1}\left(z ; \xi_{1}^{\left(m^{\prime \prime}\right)}\right)=\frac{\left(\xi_{1}^{\left(m^{\prime}\right)}+\xi_{1}^{\left(m^{\prime \prime}\right)}\right) C_{1}\left(z ; \xi_{1}^{\left(m^{\prime}\right)}\right)+\xi_{1}^{\left(m^{\prime}\right)}-\xi_{1}^{\left(m^{\prime \prime}\right)}}{\left(\xi_{1}^{\left(m^{\prime}\right)}-\xi_{1}^{\left(m^{\prime \prime}\right)}\right) C_{1}\left(z ; \xi_{1}^{\left(m^{\prime}\right)}\right)+\xi_{1}^{\left(m^{\prime}\right)}+\xi_{1}^{\left(m^{\prime \prime}\right)}}, \quad z \in \mathbb{D} .
$$

It is easy to see that both sides of (8.21) are meromorphic functions and the number of poles of the function $C_{1}\left(z ; \xi_{1}^{\left(m^{\prime \prime}\right)}\right)$ is $N_{1}\left(\xi_{1}^{\left(m^{\prime \prime}\right)}\right)$, but the number of poles of the function in the right-hand side of (8.21) does not exceed $N_{1}\left(\xi_{1}^{\left(m^{\prime}\right)}\right)$. Hence we arrive at a contradiction.

Consider the second case. Here we may write $l$ as $l=N_{j} N_{j}^{*}, j=1,2$. Without loss of generality, we assume that $\left\{e^{\theta_{1, m, j}}, \ldots, e^{\theta_{N_{j}^{*}, m, j}}\right\}$, where $0<\theta_{1, m, j}<\cdots<$ $\theta_{N_{j}^{*}, m, j}<2 \pi$, are poles of $C_{j}\left(z ; \xi_{j}^{(m)}\right)$. We see that $-i C_{j}^{\prime}\left(e^{i \theta} ; \xi_{j}^{(m)}\right)>0$ for $\theta \in$ $[0,2 \pi) \backslash\left\{\theta_{1, m, j}, \ldots, \theta_{N_{j}^{*}, m, j}\right\}$. Furthermore, $\theta \downarrow \theta_{s, m, j}$ implies $C_{j}\left(e^{i \theta} ; \xi_{j}^{(m)}\right) \uparrow-\infty$ for $s=1, \ldots, N_{j}^{*}$ and $\theta \uparrow \theta_{s, m, j}$ implies $C_{j}\left(e^{i \theta} ; u_{j}^{(m)}\right) \downarrow+\infty$ for $s=1, \ldots, N_{j}^{*}$.

Let us assume that one of $N_{1}$ or $N_{2}$, say $N_{1}$, is not equal to 2 . Then $N_{1}^{*} \leqslant l / 3$ and $N_{2}^{*} \leqslant l / 2$. Since $Z_{j}(z)=C_{j}(z ; \xi) /\left(\xi\left(C_{j}(z ; \xi)+1\right)\right)$, we note by (8.19) that 
the number of poles of the functions $Z_{1}(z), Z_{2}(z)$ are equal to $N_{1}^{*}, N_{2}^{*}$, respectively. Return to the relation (8.15). We see that the number of poles of the function $Z_{1}(z) Z_{2}(z)$ does not exceed $5 l / 6$, but, as it is easy to see, the number of poles of the function $Q_{\mu_{1}}\left(Z_{1}(z)\right)$ is equal to $l$, a contradiction.

Consider the case $N_{1}=N_{2}=2$. In this case we obtain from (8.20)

$$
q_{1,1} C_{1}\left(z ; \xi_{1}^{(1)}\right)+q_{2,1} C_{1}\left(z ; \xi_{1}^{(2)}\right)=q_{1,2} C_{2}\left(z ; \xi_{2}^{(1)}\right)+q_{2,2} C_{2}\left(z ; \xi_{2}^{(2)}\right), \quad z \in \mathbb{C} .
$$

As shown above the meromorphic functions $C_{j}\left(z ; \xi_{j}^{(1)}\right)$ and $C_{j}\left(z ; \xi_{j}^{(2)}\right)$ have no common poles and union of their poles coincides with the set of poles of the meromorphic function $\psi_{\mu}(z)$. By (8.21), the poles of $C_{1}\left(z ; \xi_{1}^{(2)}\right)$ coincides with zeros of the function $C_{1}\left(z ; \xi_{1}^{(1)}\right)+\left(\xi_{1}^{(1)}+\xi_{1}^{(2)}\right) /\left(\xi_{1}^{(1)}-\xi_{1}^{(2)}\right)$.

Using the information about the behaviour of $C_{1}\left(z ; \xi_{1}^{(1)}\right)$ on the circle $\mathbb{T}$, we see that the poles of the functions $C_{1}\left(z ; \xi_{1}^{(1)}\right)$ and $C_{1}\left(z ; \xi_{1}^{(2)}\right)$ alternate. A similar result holds for the functions $C_{2}\left(z ; \xi_{2}^{(j)}\right), j=1,2$. Hence we may conclude that the poles of $C_{1}\left(z ; \xi_{1}^{(k)}\right), k=1,2$, coincide with the poles of $C_{2}\left(z ; \xi_{2}^{(1)}\right)$ or $C_{2}\left(z ; \xi_{2}^{(2)}\right)$, say $C_{2}\left(z ; \xi_{2}^{(k)}\right)$. Then we deduce from (‥22) that

$$
q_{1,1} C_{1}\left(z ; \xi_{1}^{(1)}\right)=q_{1,2} C_{2}\left(z ; \xi_{2}^{(1)}\right) \quad \text { and } \quad q_{2,1} C_{1}\left(z ; \xi_{1}^{(2)}\right)=q_{2,2} C_{2}\left(z ; \xi_{2}^{(2)}\right), \quad z \in \mathbb{C} .
$$

Since $Z_{1}(0)=Z_{2}(0)=0$, we have $C_{k}\left(0 ; \xi_{k}^{(l)}\right)=1$ for $k, l=1,2$, and we obtain from the previous relations the equalities $q_{1,1}=q_{1,2}$ and $q_{2,1}=q_{2,2}$. Hence these relations imply that

$$
Z_{1}(z)=\frac{\xi_{2}^{(1)}}{\xi_{1}^{(1)}} Z_{2}(z)=\frac{\xi_{2}^{(2)}}{\xi_{1}^{(2)}} Z_{2}(z), \quad z \in \mathbb{C} .
$$

Therefore the formula 8.15) may be written as

$$
\xi_{0} Z_{2}^{2}(z)=z Q_{\mu_{1}}\left(Z_{1}(z)\right)=z \frac{\psi_{\mu_{1}}\left(Z_{1}(z)\right)}{1+\psi_{\mu_{1}}\left(Z_{1}(z)\right)}, \quad z \in \mathbb{C} .
$$

where $\xi_{0} \in \mathbb{T}$. Note that zeros of the function $Q_{\mu_{1}}\left(Z_{1}(z)\right)$ coincide with the zeros of $\psi_{\mu_{1}}\left(Z_{1}(z)\right)$ and are thereby simple. But from the left-hand side of (8.23) it follows that the function $\psi_{\mu_{1}}\left(Z_{1}(z)\right)$ has multiple zeros, a contradiction.

Hence for one of $j=1,2$, say $j=1$, we have $N_{1}=1$ or $N_{l}=l$. Consider the case $N_{1}=l$. Here relation (8.19) for $j=1$ has the form

$$
C_{1}\left(z ; \xi_{1}^{(m)}\right)=\frac{1+Z_{1}(z) \xi_{1}^{(m)}}{1-Z_{1}(z) \xi_{1}^{(m)}}=p_{k(m), 1}\left(\xi_{1}^{(m)}\right) \frac{1+z \xi_{k(m)}}{1-z \xi_{k(m)}}, \quad m=1, \ldots, l .
$$

where $k\left(m^{\prime}\right) \neq k\left(m^{\prime \prime}\right)$ if $m^{\prime} \neq m^{\prime \prime}$. Since $C_{1}\left(0 ; \xi_{1}^{(m)}\right)=0$, we obtain $p_{k(m), 1}\left(\xi_{1}^{(m)}\right)=$ $1, m=1, \ldots, l$. From (8.24) it follows that $Z_{1}(z)=z \xi_{k(m)} / \xi^{(m)}$ for all $m=$ $1, \ldots, l$. Hence $Z_{1}(z)=z \xi_{0}$ for some $\xi_{0} \in \mathbb{T}$ and we obtain $\psi_{\mu}(z)=\psi_{\mu_{1}}\left(z \xi_{0}\right), z \in$ D. We finally conclude that $\mu=\mu_{1} \otimes \delta_{\xi_{0}}$. This means that $\mu_{1}=\mu_{\bigotimes} \delta_{1 / \xi_{0}}$ and $\mu_{2}=\delta_{\xi_{0}}$. 
It remains to consider the case $N=1$ where obviously $\mu_{1}=\delta_{\xi_{0}}$ holds for some $\xi_{0} \in \mathbb{T}$ and hence $\mu_{2}=\mu_{\bigotimes} \delta_{1 / \xi_{0}}$.

Thus, Theorem 2.14 is completely proved.

\section{REFERENCES}

[1] Akhiezer, N. I. , The classical moment problem and some related questions in analysis, Hafner, New York, 1965.

[2] Akhiezer, N. I. and Glazman, I. M. , Theory of Linear Operators in Hilbert Space, Ungar, New York, 1963.

[3] Bercovici, H., and Voiculescu, D. , Lévy-Hinčin type theorems for multiplicative and additive free convolution, Pacific J. Math., 153 (1992), 217-248.

[4] Bercovici, H., and Voiculescu, D. , Free convolution of measures with unbounded support, Indiana Univ. Math. J., 42 (1993), 733-773.

[5] Bercovici, H., and Voiculescu, D. , Superconvergence to the central limit and failure of the Cramér theorem for free random variables, Probab. Theory Relat. Fields, 102 (1995), 215-222.

[6] Bercovici, H., and Pata, V. , Stable laws and domains of attraction in free probability theory, Annals of Math.,149, (1999), 1023-1060.

[7] Bercovici, H., and Pata, V. , A free analogue of Hinčin's characterization of infinitely divisibility, Proc. AMS, 128, (2000), 1011-1015.

[8] Biane, Ph., Processes with free increments, Math. Z., 227 (1998), 143-174.

[9] Davidson, R. , Arithmetic and other properties of certain Delphic semigroups,I, Z. Wahrscheinlichkeitstheory verw. Geb., 10 (1968), 120-145.

[10] Davidson, R. , Arithmetic and other properties of certain Delphic semigroups,II, Z. Wahrscheinlichkeitstheory verw. Geb. 10 (1968),146-172.

[11] Davidson, R., More Delphic theory and practice, Z. Wahrscheinlichkeitstheory verw. Geb., 13 (1969), 191-203.

[12] Goluzin, G. M., Geometric Theory of Functions od a Compex Variable, Amer. Math. Soc., Providence, Rhode Island 02904, 1969.

[13] Kendall, D. G. , Delphic semigroups, Bull. Amer. math. Soc., 73 (1967), 120-121.

[14] Kendall, D. G. , Delphic semigroups, infinitely divisible regenerative phenomena, and the arithmetic of p-functions, Z. Wahrscheinlichkeitstheory verw. Geb., 9 (1968), 163-195.

[15] Khintchine, A. Ya., The arithmetic of distribution laws, Bull. Univ. État Moscou, Sér. Int., Sect. A: Math. et Mécan., 1 (1937), no. 1, 6-17.

[16] Linnik, Yu.V. and Ostrovskii, I.V., Decomposition of Random Variables and Vectors Amer. Math. Soc., Providence, Rhode Island, 1977, pp. 380.

[17] Livshic, L.Z., Ostrovskii, I.V. and Chistyakov, G.P. , The arithmetic of probality laws. (Russian), Probability theory, Mathematical statistic, Theoretical cybernatikcs, 12 (1975), Akad. Nauk SSSR Vsesojuz. Inst. Nauch. i Tehn. Informacii, Moscow, 5-42.

[18] Maassen, H. , Addition of Freely Independent Random Variables, Journal of functional analysis, 106 (1992), 409-438.

[19] Markushevich, A., Theory of functions of a complex variable, Prentice-Hall, INC, 2, 1965; 3, 1967.

[20] Nevanlinna, R. and Paatero, V., Introduction to Complex Analysis, Addison-Wesley Publishing Company, 1969. 
[21] Ostrovskii, I.V., The arithmetic of probability distributions, J. Multivariate Anal., 7 (1977), no. $4,475-490$.

[22] Ostrovskii, I.V. , The arithmetic of probability distributions, Theory Probab. Appl., 31 (1986), no. 1, 3-30.

[23] Pastur, L. and Vasilchuk, V., On the Law of Addition of Random Matrices, Commun. Math. Phys., 214 (2000), 249-286.

[24] Speicher, R. and Woroudi, R. Boolean Convolution, Fields Ins. Communications, 12 (1997), 267-279.

[25] Speicher, R. , Combinatorical theory of the free product with amalgamation and operatorvalued free probability theory, Mem. A.M.S., 627 (1998).

[26] Vasilchuk, V. , On the Law of Multiplication of Random Matrices, Math. Phys. Anal. Geom., 4 (2001), no. 4, 1-36.

[27] Voiculescu, D.V. , Addition of certain noncommuting random variavles, J. Funct. Anal., 66 (1986), 323-346.

[28] Voiculescu, D.V., Multiplication of certain noncommuting random variavles, J. Operator Theory, 18 (1987), 223-235.

[29] Voiculesku, D., Dykema, K., and Nica, A. , Free random variables, CRM Monograph Series, No 1, A.M.S., Providence, RI, 1992.

Gennadii Chistyakov, Institute for Low Temperature Physics and Engineering, National Academy of Sciences of Ukraine, 47 Lenin Ave. 61103 Kharkov, Ukraine chistyakov@ilt.kharkov.ua, chistyak@mathematik.uni-bielefeld.de

Friedrich Götze, Mathematics Department, Bielefeld University, Postfach 100131, 33501 Bielefeld, Germany

goetze@mathematik.uni-bielefeld.de 